\title{
ERGODICITY OF UNIPOTENT FLOWS AND KLEINIAN GROUPS
}

\author{
AMIR MOHAMMADI AND HEE OH
}

\section{Contents}

1. Introduction

2. Ergodic properties of BMS and BR measures 537

3. Weak convergence of the conditional of $\mu_{E, s}^{\mathrm{BR}}$

4. PS density and its non-focusing property when $\delta>1$

5. Energy estimate and $L^{2}$-convergence for the projections

6. Recurrence properties of BMS and BR measures

7. Window theorem for Hopf average $\quad 559$

8. Additional invariance and ergodicity of BR for $\delta>1$

9. $\quad$ BR is not ergodic if $0<\delta \leq 1$

Acknowledgments $\quad 576$

\begin{tabular}{ll} 
References & 576 \\
\hline
\end{tabular}

\section{INTRODUCTION}

In this paper we study dynamical properties of one-parameter unipotent flow for the frame bundle of a convex cocompact hyperbolic 3-manifold $\mathcal{M}$. When the critical exponent of the fundamental group $\pi_{1}(\mathcal{M})$ exceeds one, we show that this flow is conservative and ergodic for the Burger-Roblin measure $m^{\mathrm{BR}}$ : almost all points enter to a given Borel subset of positive measure for an unbounded amount of time. Such a manifold admits a unique positive square-integrable eigenfunction $\phi_{0}$ of the Laplacian with base eigenvalue. Our result implies that a randomly chosen unipotent orbit, normalized by the time average of the eigenfunction $\phi_{0}$, becomes equidistributed with respect to the Burger-Roblin measure.

To state our result more precisely, let $G=\mathrm{PSL}_{2}(\mathbb{C})$, which is the group of orientation preserving isometries of the hyperbolic space $\mathbb{H}^{3}$. Let $\Gamma$ be a non-elementary, torsion-free, discrete subgroup of $G$ which is convex cocompact, that is, the convex core of $\Gamma$ is compact. Equivalently, $\Gamma \backslash \mathbb{H}^{3}$ admits a finite sided fundamental domain with no cusps. Convex cocompact groups arise in topology as fundamental groups of compact hyperbolic 3-manifolds with totally geodesic boundary.

Received by the editors September 15, 2012 and, in revised form, February 23, 2014.

2010 Mathematics Subject Classification. Primary 11N45, 37F35, 22E40; Secondary 37A17, $20 \mathrm{~F} 67$.

Key words and phrases. Geometrically finite hyperbolic groups, Ergodicity, Burger-Roblin measure, Bowen-Margulis-Sullivan measure.

The first author was supported in part by NSF Grant \#1200388.

The second author was supported in part by NSF Grant \#1068094. 
The frame bundle of the manifold $\mathcal{M}=\Gamma \backslash \mathbb{H}^{3}$, which is a circle bundle over the unit tangent bundle $\mathrm{T}^{1}(\mathcal{M})$, is identified with the homogeneous space $X=\Gamma \backslash G$. We consider the unipotent flow on $X$ given by the right translations of the oneparameter unipotent subgroup

$$
U=\left\{u_{t}:=\left(\begin{array}{ll}
1 & 0 \\
t & 1
\end{array}\right): t \in \mathbb{R}\right\} .
$$

This flow is called ergodic, with respect to a fixed locally finite Borel measure on $X$, if any invariant Borel subset is either null or co-null. We denote by $\delta$ the critical exponent of $\Gamma$, which is equal to the Hausdorff dimension of the limit set of $\Gamma$ [34]. When $\delta=2, X$ is compact [37] and the classical Moore's theorem in 1966 [23] implies that this flow is ergodic with respect to the volume measure, i.e., the $G$ invariant measure. When $\delta<2$, the volume measure is not ergodic any more, and furthermore, Ratner's measure classification theorem [30] says that there exists no finite $U$-ergodic invariant measure on $X$. This raises a natural question of finding a locally finite $U$-ergodic measure on $X$. Our main result in this paper is that when $\delta>1$, the Burger-Roblin measure is conservative and ergodic, and is never ergodic otherwise.

The conservativity means that for any subset $S$ of positive measure, the $U$-orbits of almost all points in $S$ spend an infinite amount of time in $S$. Any finite invariant measure is conservative by the Poincaré recurrence theorem. For a general locally finite invariant measure, the Hopf decomposition theorem [14] says that any ergodic measure is either conservative or totally dissipative (i.e., for any Borel subset $S$, $x u_{t} \notin S$ for all large $|t| \gg 1$ and a.e. $\left.x \in S\right)$. For $\delta<2$, there are many isometric embeddings of the real line in $X$, by $t \mapsto x u_{t}$, giving rise to a family of dissipative ergodic measures for $U$.

We refer to the Burger-Roblin measure as the BR measure for short, and give its description using the Iwasawa decomposition $G=K A N: K=\mathrm{PSU}_{2}, A=\left\{a_{s}\right.$ : $s \in \mathbb{R}\}, N=\left\{n_{z}: z \in \mathbb{C}\right\}$ where

$$
a_{s}=\left(\begin{array}{cc}
e^{s / 2} & 0 \\
0 & e^{-s / 2}
\end{array}\right) \text { and } n_{z}=\left(\begin{array}{cc}
1 & 0 \\
z & 1
\end{array}\right)
$$

Furthermore let $M$ denote the centralizer of $A$ in $K$.

The groups $A$ and $N$ play important roles in dynamics as the right translation by $a_{s}$ on $X$ is the frame flow, which is the extension of the geodesic flow on $\mathrm{T}^{1}(\mathcal{M})$ and $N$-orbits give rise to unstable horospherical foliation on $X$ for the frame flow.

Fixing $o \in \mathbb{H}^{3}$ stabilized by $K$, we denote by $\nu_{o}$ the Patterson-Sullivan measure on the boundary $\partial\left(\mathbb{H}^{3}\right)$, supported on the limit set of $\Gamma$, associated to $\left.o([26], 34]\right)$, and refer to it as the PS measure. Sullivan showed that the PS measure coincides with the $\delta$-dimensional Hausdorff measure of the limit set of $\Gamma$. Using the transitive action of $K$ on $\partial\left(\mathbb{H}^{3}\right)=K / M$, we may lift $\nu_{o}$ to an $M$-invariant measure on $K$.

Burger-Roblin measure Define the measure $\tilde{m}^{\text {BR }}$ on $G$ as follows: for $\psi \in C_{c}(G)$,

$$
\tilde{m}^{\mathrm{BR}}(\psi)=\int_{G} \psi\left(k a_{s} n_{z}\right) e^{-\delta s} d \nu_{o}(k) d s d z
$$

where $d s$ and $d z$ are the Lebesgue measures on $\mathbb{R}$ and $\mathbb{C}$ respectively. It is left $\Gamma$-invariant and right $N$-invariant. The BR measure $m^{\mathrm{BR}}$ is a locally finite measure 
on $X$ induced by $\tilde{m}^{\mathrm{BR}}$. When $\delta=2, \mathrm{~m}^{\mathrm{BR}}$ is simply a $G$-invariant measure, but it is an infinite measure if $\delta<2$.

Roblin showed that the BR measure is the unique $N M$-invariant ergodic measure on $X$ which is not supported on a closed $N M$-orbit in $X$ 31. For $\Gamma$ Zariski dense (which is the case if $\delta>1$ ), Winter [36] proved that $m^{\mathrm{BR}}$ is $N$-ergodic, and this implies that $m^{\mathrm{BR}}$ is the unique $N$-invariant ergodic measure on $X$ which is not supported on a closed $N$-orbit in $X$, by Roblin's classification. We note that the analogous result for $G=\mathrm{PSL}_{2}(\mathbb{R})$ was established earlier by Burger [5] when $\Gamma$ is convex-compact with $\delta>1 / 2$.

The main result of this paper is:

Theorem 1.1. Let $\Gamma$ be a convex cocompact subgroup of $G$ which is not virtually abelian. The $U$-flow on $\left(X, m^{\mathrm{BR}}\right)$ is ergodic if and only if $\delta>1$.

We also show the conservativity of the BR-measure for $\delta>1$, without knowing its ergodicity a priori. Indeed we establish the non-ergodicity in the case $0<\delta \leq 1$ by proving the failure of "sufficient" recurrence; see Section 9 .

Remark 1.2. We remark that most of arguments in the proof of Theorem 1.1 works for a higher dimensional case as well. Namely, the same proof will show that if $G$ is the group of orientation preserving isometries of the hyperbolic $n$-space, $\Gamma$ is a Zariski dense, convex cocompact subgroup of $G$, and $U$ is a $k$-dimensional connected unipotent subgroup of $G$, then the $U$ action is ergodic with respect to the BR-measure on $\Gamma \backslash G$ if $\delta>n-k$ and not ergodic if $\delta<n-k$. The case of $\delta=n-k$ is more subtle. Our argument for $n=3$ uses certain known facts about the limit sets of Kleinian groups of $\mathrm{PSL}_{2}(\mathbb{C})$; see section 9 .

For a probability measure $\mu$ on $X$, the Birkhoff pointwise ergodic theorem (1931) says that the ergodicity of a measure preserving flow $\left\{u_{t}\right\}$ implies that the time average of a typical orbit converges to the space average: for any $\psi \in L^{1}(X)$ and a.e. $x \in X$, as $T \rightarrow \infty$,

$$
\frac{1}{T} \int_{0}^{T} \psi\left(x u_{t}\right) d t \longrightarrow \int_{X} \psi d \mu .
$$

A generalization of the Birkhoff theorem for an infinite locally finite conservative ergodic measure was obtained by E. Hopf [11] in 1937 and says that the ratio of time averages of a typical orbit for two functions converges to the ratio of the space averages: for any $\psi_{1}, \psi_{2} \in L^{1}(X)$ with $\psi_{2} \geq 0$ with $\int_{X} \psi_{2} d \mu>0$, as $T \rightarrow \infty$,

$$
\frac{\int_{0}^{T} \psi_{1}\left(x u_{t}\right) d t}{\int_{0}^{T} \psi_{2}\left(x u_{t}\right) d t} \longrightarrow \frac{\int_{X} \psi_{1} d \mu}{\int_{X} \psi_{2} d \mu} \quad \text { a.e. } x \in X .
$$

For our $X=\Gamma \backslash G$ with $\Gamma$ convex cocompact and $\delta>1$, there is a unique positive eigenfunction $\phi_{0} \in L^{2}(\mathcal{M})$ for the Laplacian with the smallest eigenvalue $\delta(2-\delta)$ and with $\left\|\phi_{0}\right\|_{2}=1$ (see [34]). In the upper half-space coordinates, $\mathbb{H}^{3}=\{z+j y$ : $z \in \mathbb{C}, y>0\}$ with $\partial\left(\mathbb{H}^{3}\right)=\mathbb{C} \cup\{\infty\}$, and the lift $\tilde{\phi}_{0}$ of $\phi_{0}$ to $\mathbb{H}^{3}$ is realized explicitly as the integral of a Poisson kernel against the PS measure $\nu_{o}($ with $o=j$ ):

$$
\tilde{\phi}_{0}(z+j y)=\int_{\xi \in \mathbb{C}}\left(\frac{\left(\|\xi\|^{2}+1\right) y}{\|z-\xi\|^{2}+y^{2}}\right)^{\delta} d \nu_{o}(\xi) .
$$


The BR measure on $X$ projects down to the absolutely continuous measure on the manifold $\mathcal{M}$ and its Radon-Nikodym derivative with respect to the hyperbolic volume measure is given by $\phi_{0}$.

We deduce the following from Theorem 1.1 and Hopf's ratio theorem (1.3):

Corollary 1.3. Let $\delta>1$.

(1) For $m^{\mathrm{BR}}$ almost all $x \in X$, the projection of $x U$ to $\mathcal{M}$ is dense.

(2) For any $\psi \in L^{1}\left(X, m^{\mathrm{BR}}\right)$ and for almost all $x \in X$,

$$
\lim _{T \rightarrow \infty} \frac{\int_{0}^{T} \psi\left(x u_{t}\right) d t}{\int_{0}^{T} \phi_{0}\left(x u_{t}\right) d t}=\int_{X} \psi d m^{\mathrm{BR}} .
$$

We explain the proof of Theorem 1.1 in the case $\delta>1$, in comparison with the finite measure case. This account makes our introduction rather lengthy but we hope that this will give a summary of the main ideas of the proof which will be helpful to the readers. The proof of Moore's ergodicity theorem is based on the following equivalence for a finite invariant measure $\mu$ : $\mu$ is ergodic if and only if any $U$-invariant function of $L^{2}(X, \mu)$ is constant a.e. Through this interpretation, his ergodicity theorem follows from a theorem in the unitary representation theory that any $U$-invariant vector in the Hilbert space $L^{2}\left(X, \mu_{G}\right)$ is $G$-invariant for the volume measure $\mu_{G}$.

For an infinite invariant measure, its ergodicity cannot be understood merely via $L^{2}$-functions, but we must investigate all invariant bounded measurable functions. This means that we cannot depend on a convenient theorem on the dual space of $X$, but rather have to work with the geometric properties of flows in the space $X$ directly. We remark that as we are working with a unipotent flow as opposed to a hyperbolic flow, the Hopf argument using the stable and unstable foliations of flows, which is a standard tool in studying the ergodicity for hyperbolic flows, is irrelevant here.

We use the polynomial divergence property of unipotent flows to establish that almost all $U$-ergodic components of $m^{\mathrm{BR}}$ are invariant under the full horospherical subgroup $N$. The $N$-ergodicity of the BR measure then implies the $U$-ergodicity as well. This approach has been noted by Margulis as an alternative approach to show the ergodicity of the volume measure $\mu_{G}$ in the finite volume case.

However, carrying out this argument in an infinite measure case is subtler. Indeed the heart of the argument, as is explained below, lies in the study of two nearby orbits in the "intermediate range". To the best of our knowledge, such questions of infinite measure spaces have not been understood before.

Let us present a sketch of the argument in the probability measure case. Let $(X, \mu)$ be a probability measure space. Then it is straightforward from (1.2) that for any generic point $x$, any $0<r<1$, and any $\psi \in C_{c}(X)$

$$
\frac{1}{(1-r) T} \int_{r T}^{T} \psi\left(x u_{t}\right) d t \rightarrow \int_{X} \psi(x) d \mu .
$$

Statements of this nature will be called a "window theorem" in the sequel.

We now explain how a suitable window theorem can be used in acquiring an additional invariance by an element of $N-U$. This idea was used by Ratner; see 29, [30] and the references therein. We also refer to [17, 18] where similar ideas were used by Margulis in the topological setting. 
Let $\check{N}$ and $\check{U}$ denote the transpose of $N$ and $U$ respectively. We denote by $N_{G}(U)$ the normalizer of $U$ in $G$.

Choose sequences of generic points $x_{k}$ and $y_{k}$ inside a suitably chosen compact subset of $X$, moreover suppose that $y_{k}=x_{k} g_{k}$ with $g_{k} \notin N_{G}(U)$ and $g_{k} \rightarrow e$. Put $\check{V}=\left\{\left(\begin{array}{cc}1 & i t \\ 0 & 1\end{array}\right): t \in \mathbb{R}\right\}$, and assume that the $\check{V}$-component and the $\check{U}$-component 1 of $g_{k}$ are of "comparable" size.

Flowing by $u_{t}$, we compare the orbits $x_{k} u_{t}$ and $y_{k} u_{t}=x_{k} u_{t}\left(u_{t}^{-1} g_{k} u_{t}\right)$. The divergence properties of unipotent flows (a simple computation in our case), in view of our above assumption on $g_{k}$ 's, imply that the divergence of the two orbits is comparable to $u_{t}^{-1} g_{k} u_{t}$. Furthermore, the $(2,1)$-matrix entry of $u_{t}^{-1} g_{k} u_{t}$ dominates other matrix entries. Let $p(t)$ denote the $(2,1)$-matrix entry of $u_{t}^{-1} g_{k} u_{t}$. This is a polynomial of degree two whose leading coefficient has comparable real and imaginary parts. Therefore, the divergence of the two orbits is "essentially" in the direction of $N-U$. Choose a sequence of times $T_{k}$ so that $p\left(T_{k}\right)$ converges to a non-trivial element $v \in N-U$. Letting $\varepsilon>0$ be small, since $p(t)$ is a polynomial, $y_{k} u_{t}$ remains within an $O(\varepsilon)$-neighborhood of $x_{k} u_{t} v$ for any $t \in\left[(1-\varepsilon) T_{k}, T_{k}\right]$. Hence the window theorem (1.4) applied to the sequence of windows $\left[(1-\varepsilon) T_{k}, T_{k}\right]$ implies that $\mu(\psi)-\mu(v \cdot \psi)=O(\varepsilon)$ and hence $\mu(\psi)=\mu(v \cdot \psi)$ as $\varepsilon>0$ is arbitrary. Repeating this process for a sequence of $v_{n} \rightarrow e$, we obtain that the measure $\mu$ is invariant under $N$.

We now turn our attention to an infinite measure case, assuming $\delta>1$. There is a subtle difference for the average over the one-sided interval $[0, T]$ and over the two sided $[-T, T]$, and the average over $[-T, T]$ is supposed to behave more typically in infinite ergodic theory. We first prove that the BR measure $m^{\mathrm{BR}}$ is $U$-conservative based on a theorem of Marstrand [19], which allows us to write an ergodic decomposition $m^{\mathrm{BR}}=\int_{x} \mu_{x}$ where $\mu_{x}$ is conservative for a.e. $x$. Letting $x$ be a generic point for Hopf's ratio theorem and $I_{T}=[-T, T]$, in order to deduce $\frac{\int_{I_{T}-I_{r T}} \psi_{1}\left(x u_{t}\right) d t}{\int_{I_{T}-I_{r T}} \psi_{2}\left(x u_{t}\right) d t} \sim \frac{\mu_{x}\left(\psi_{1}\right)}{\mu_{x}\left(\psi_{2}\right)}$, it is sufficient to prove that there is some $c>0$ such that for all $T \gg 1$,

$$
\int_{I_{T}-I_{r T}} \psi_{2}\left(x u_{t}\right) d t \geq c \int_{I_{T}} \psi_{2}\left(x u_{t}\right) d t .
$$

This type of inequality requires strong control on the recurrence of the flow, and seems unlikely that (1.5) can be achieved for a set of positive measure, see 11, Section 2.4]. Hence formulating a proper replacement of this condition (1.5) and its proof are simultaneously the hardest part and at the heart of the proof of Theorem 1.1.

We call $x \in X$ a BMS point if both the forward and backward endpoints of the geodesic determined by $x$ belong to the limit set of $\Gamma$. These points precisely comprise the support of the Bowen-Margulis-Sullivan measure $m^{\text {BMS }}$ on $X$, which is the unique measure of maximal entropy for the geodesic flow, up to a multiplicative constant (see Section 2.3). We will call $m^{\mathrm{BMS}}$ the BMS measure for simplicity. The support of $m^{\mathrm{BMS}}$ is contained in the convex core of $\Gamma$, and in particular a compact subset. By a BMS box, we mean a subset of the form $x_{0} \check{N}_{\rho} A_{\rho} N_{\rho} M$ where $x_{0} \in X$ is a BMS point, $\rho>0$ is at most the injectivity radius at $x_{0}$ and $S_{\rho}$ means the $\rho$-neighborhood of $e$ in $S$ for any $S \subset G$.

\footnotetext{
${ }^{1}$ these components are well defined for all $g_{k}$ close enough to $e$.
} 
Theorem 1.4 (Window Theorem). Let $\delta>1$. Let $E \subset X$ be a BMS box and $\psi \in C_{c}(X)$ be a non-negative function with $\left.\psi\right|_{E}>0$. Then there exist $0<r<1$ and $T_{0}>1$ such that for any $T \geq T_{0}$,

$$
m^{\mathrm{BR}}\left\{x \in E: \int_{-r T}^{r T} \psi\left(x u_{t}\right) d t \leq(1-r) \int_{-T}^{T} \psi\left(x u_{t}\right) d t\right\}>\frac{r}{2} \cdot m^{\mathrm{BR}}(E) .
$$

We call $x$ a good point for the window $I_{T}-I_{r T}$ if

$$
\int_{I_{r T}} \psi\left(x u_{t}\right) d t \leq(1-r) \int_{I_{T}} \psi\left(x u_{t}\right) d t
$$

or equivalently if $\int_{I_{T}-I_{r T}} \psi\left(x u_{t}\right) d t \geq r \int_{I_{T}} \psi\left(x u_{t}\right) d t$. The window theorem says that the set of good points for the window $I_{T}-I_{r T}$ has a positive proportion of $E$ for all large $T$. It follows that for any $\varepsilon>0$, we can choose a sequence $T_{k}=T_{k}(\varepsilon)$ such that the set $E_{k}$, of good points for the window $\left[(1-\varepsilon) T_{k}, T_{k}\right]$ (or $\left.\left[-T_{k},-(1-\varepsilon) T_{k}\right]\right)$, has positive measure. Let $x_{k}, y_{k}=x_{k} g_{k} \in E_{k}$. To be able to use this in obtaining an additional invariance, we need to control the size as well as the direction of the divergence $u_{T_{k}}^{-1} g_{k} u_{T_{k}}$. More precisely, we need to be able to choose our generic points $y_{k}=x_{k} g_{k}$ so that the size of $g_{k}$ is comparable with $\frac{1}{T_{k}^{2}}$ and the size of its $\check{V}$-component is comparable with that of $\check{U}$-component.

We emphasize here that we work in the opposite order of a standard way of applying the pointwise ergodic theorem where one is usually given a sequence $g_{k}$ and then find window $I_{T_{k}}$ depending on $g_{k}$ (as the window theorem works for any $T_{k}$ ). In our situation, we cannot choose $T_{k}$, but rather have to work with given $T_{k}$ (depending on $\varepsilon$ ). So, only after we know which $T_{k}$ 's give good windows for the $\varepsilon$-width, we can choose good points $x_{k} g_{k}$ for those windows. What allows us to carry out this process is that we have a good understanding of the structure of the generic set along contracting leaves. To be more precise, the PS-measures on the contracting leaves are basically $\delta$-dimensional Hausdorff measures on $\mathbb{R}^{2}$, and the assumption that $\delta>1$ enables us to find $g_{k}$ for the "right scale", see Section 4 .

Hoping to have given some idea about how the above window theorem 1.4 will be used, we now discuss its proof, which is based on the interplay between the BR measure and the BMS measure. We mention that the close relationship between the $\mathrm{BR}$ and the BMS measure is also the starting point of Roblin's unique ergodicity theorem for $N M$-invariant measures.

Unlike the finite measure case, $m^{\mathrm{BR}}$ is not invariant under the frame flow, which is the right translation by $a_{s}$ in $X$. However, as $s \rightarrow+\infty$, the normalized measure $\mu_{s}^{\mathrm{BR}}:=\left.\left(a_{-s}\right)_{*} m^{\mathrm{BR}}\right|_{E}$ (the push-forward of the restriction $\left.m^{\mathrm{BR}}\right|_{E}$ by the frame flow $\left.a_{-s}\right)$ converges to $m^{\mathrm{BMS}}$ in the weak* topology.

Under the assumption $\delta>1$, the BMS measure turns out to be $U$-recurrent and hence almost all of its $U$-leafwise measures are non-atomic. This will imply that the analogue of (1.5) holds for "most" of the $U$-leafwise measures of $m^{\text {BMS }}$.

The goal is to utilize this and the fact that $\mu_{s}^{\mathrm{BR}}$ weakly converges to $m^{\mathrm{BMS}}$, in order to deduce that many of the $U$-leafwise measures of $m^{\text {BR }}$ must also satisfy (1.5). We mention that, in general, it is unusual to be able to deduce "interesting" statements regarding leafwise measures from weak* convergence of measures. One possible explanation for this is that the leafwise measures of a sequence of measures may change "very irregularly" as one moves in the transversal direction, e.g. approximation of Lebesgue measure by atomic measures. 
We succeed here essentially because we have a rather good understanding of the $N$-leafwise measures of $\mu_{s}^{\mathrm{BR}}$. To be more precise, we can show (i) the $N$-leafwise measures of $\mu_{s}^{\mathrm{BR}}$ change rather regularly (see Section 3) furthermore, (ii) the projection of an $N$-leafwise measure of $\mu_{s}^{\mathrm{BR}}$ converges in the $L^{2}$-sense to its counterpart of $m^{\mathrm{BMS}}$ in most directions, see Section 5.1

We emphasize that we established the $L^{2}$-convergence of these measures, not merely the weak* convergence, and this is crucial to our proof; see the Key Lemma 5.12 and Section 7 . The proof of this $L^{2}$-convergence requires a certain control of the energy of the conditional measures of $\mu_{s}^{\mathrm{BR}}$ which is uniform for all $s \gg 1$. Our energy estimate is obtained using the following deep property of the PS measure: for all $\xi$ in the limit set of $\Gamma$ and for all small $r>0, \nu_{o}(B(\xi, r)) \asymp r^{\delta}$ (with the implied constant being independent of $\xi$ and $r$ ), together with the Besicovitch covering lemma. Lastly, we remark that our proof of the window theorem makes use of the rich theory of entropy and is inspired by the low entropy method developed by Lindenstrauss in [15].

\section{ERGOdIC PROPERTIES OF BMS AND BR MEASURES}

\subsection{Measures on $\mathbf{T}^{1}\left(\Gamma \backslash \mathbb{H}^{3}\right)$ associated to a pair of conformal densities.} Let $\left(\mathbb{H}^{3}, d\right)$ denote the hyperbolic 3 -space and $\partial\left(\mathbb{H}^{3}\right)$ its geometric boundary. We denote by $\mathrm{T}^{1}\left(\mathbb{H}^{3}\right)$ the unit tangent bundle of $\mathbb{H}^{3}$ and by $\pi$ the natural projection from $\mathrm{T}^{1}\left(\mathbb{H}^{3}\right) \rightarrow \mathbb{H}^{3}$.

Denote by $\left\{g^{s}: s \in \mathbb{R}\right\}$ the geodesic flow. For $u \in \mathrm{T}^{1}\left(\mathbb{H}^{3}\right)$, we set

$$
u^{+}:=\lim _{s \rightarrow \infty} g^{s}(u) \quad \text { and } \quad u^{-}:=\lim _{s \rightarrow-\infty} g^{s}(u)
$$

which are respectively the forward and backward endpoints in $\partial\left(\mathbb{H}^{3}\right)$ of the geodesic defined by $u$.

Definition 2.1. (1) The Busemann function $\beta: \partial\left(\mathbb{H}^{3}\right) \times \mathbb{H}^{3} \times \mathbb{H}^{3} \rightarrow \mathbb{R}$ is defined as follows: for $\xi \in \partial\left(\mathbb{H}^{3}\right)$ and $x, y \in \mathbb{H}^{3}$,

$$
\beta_{\xi}(x, y)=\lim _{s \rightarrow \infty} d\left(x, \xi_{s}\right)-d\left(y, \xi_{s}\right)
$$

where $\xi_{s}$ is a geodesic ray tending to $\xi$ as $s \rightarrow \infty$ from a base point $o \in \mathbb{H}^{3}$, fixed once and for all.

(2) For $u \in \mathrm{T}^{1}\left(\mathbb{H}^{3}\right)$, the unstable horosphere $\mathcal{H}_{u}^{+}$and the stable horosphere $\check{\mathcal{H}}_{u}$ denote respectively the subsets

$$
\begin{aligned}
& \left\{v \in \mathrm{T}^{1}\left(\mathbb{H}^{3}\right): v^{-}=u^{-}, \beta_{u^{-}}(\pi(u), \pi(v))=0\right\} \\
& \left\{v \in \mathrm{T}^{1}\left(\mathbb{H}^{3}\right): v^{+}=u^{+}, \beta_{u^{+}}(\pi(u), \pi(v))=0\right\} .
\end{aligned}
$$

Each element of the group $\operatorname{PSL}_{2}(\mathbb{C})$ acts on $\hat{\mathbb{C}}=\mathbb{C} \cup\{\infty\}$ as a Mobius transformation and its action extends to an isometry of $\mathbb{H}^{3}$, giving the identification of $\mathrm{PSL}_{2}(\mathbb{C})$ as the group of orientation preserving isometries of $\mathbb{H}^{3}$. Note that $(g(u))^{ \pm}=g\left(u^{ \pm}\right)$for $g \in G$. The map $\mathrm{T}^{1}\left(\mathbb{H}^{3}\right) \rightarrow \partial\left(\mathbb{H}^{3}\right)$ given by $u \mapsto u^{+}$is called the visual map.

For discussions in this section, we refer to [31, [24] and [22]. Let $\Gamma$ be a nonelementary (i.e., non virtually abelian) torsion-free discrete subgroup of $G$. Let $\left\{\mu_{x}: x \in \mathbb{H}^{3}\right\}$ be a $\Gamma$-invariant conformal density of dimension $\delta_{\mu}>0$ on $\partial\left(\mathbb{H}^{3}\right)$. 
That is, each $\mu_{x}$ is a non-zero finite Borel measure on $\partial\left(\mathbb{H}^{3}\right)$ satisfying for any $x, y \in \mathbb{H}^{3}, \xi \in \partial\left(\mathbb{H}^{3}\right)$ and $\gamma \in \Gamma$,

$$
\gamma_{*} \mu_{x}=\mu_{\gamma x} \quad \text { and } \quad \frac{d \mu_{y}}{d \mu_{x}}(\xi)=e^{-\delta_{\mu} \beta_{\xi}(y, x)},
$$

where $\gamma_{*} \mu_{x}(F)=\mu_{x}\left(\gamma^{-1}(F)\right)$ for any Borel subset $F$ of $\partial\left(\mathbb{H}^{3}\right)$.

Let $\left\{\mu_{x}\right\}$ and $\left\{\mu_{x}^{\prime}\right\}$ be $\Gamma$-invariant conformal densities on $\partial\left(\mathbb{H}^{3}\right)$ of dimension $\delta_{\mu}$ and $\delta_{\mu^{\prime}}$ respectively. Following Roblin [31, we define a measure $m^{\mu, \mu^{\prime}}$ on $\mathrm{T}^{1}\left(\Gamma \backslash \mathbb{H}^{3}\right)$ associated to the pair $\left\{\mu_{x}\right\}$ and $\left\{\mu_{x}^{\prime}\right\}$. Note that, fixing $o \in \mathbb{H}^{3}$, the map

$$
u \mapsto\left(u^{+}, u^{-}, \beta_{u^{-}}(o, \pi(u))\right)
$$

is a homeomorphism between $\mathrm{T}^{1}\left(\mathbb{H}^{3}\right)$ with $\left(\partial\left(\mathbb{H}^{3}\right) \times \partial\left(\mathbb{H}^{3}\right)-\left\{(\xi, \xi): \xi \in \partial\left(\mathbb{H}^{3}\right)\right\}\right) \times \mathbb{R}$.

Definition 2.2. Set

$$
d \tilde{m}^{\mu, \mu^{\prime}}(u)=e^{\delta_{\mu} \beta_{u^{+}}(o, \pi(u))} e^{\delta_{\mu^{\prime}} \beta_{u^{-}}(o, \pi(u))} d \mu_{o}\left(u^{+}\right) d \mu_{o}^{\prime}\left(u^{-}\right) d t .
$$

It follows from the $\Gamma$-conformal properties of $\left\{\mu_{x}\right\}$ and $\left\{\mu_{x}^{\prime}\right\}$ that $\tilde{m}^{\mu, \mu^{\prime}}$ is $\Gamma$ invariant and that this definition is independent of the choice of $o \in \mathbb{H}^{3}$. Therefore, it induces a locally finite Borel measure $m^{\mu, \mu^{\prime}}$ on $\mathrm{T}^{1}\left(\Gamma \backslash \mathbb{H}^{3}\right)$.

2.2. BMS and BR measures on $\mathbf{T}^{1}\left(\Gamma \backslash \mathbb{H}^{3}\right)$. Two important densities we will consider are the Patterson-Sullivan density and the $G$-invariant density.

We denote by $\delta$ the critical exponent of $\Gamma$, that is, the abscissa of convergence of the Poincare series $\mathcal{P}_{\Gamma}(s):=\sum_{\gamma \in \Gamma} e^{-s d(o, \gamma(o))}$ for $o \in \mathbb{H}^{3}$. As $\Gamma$ is non-elementary, we have $\delta>0$. The limit set $\Lambda(\Gamma)$ is the set of all accumulation points of orbits $\Gamma(z)$, $z \in \mathbb{H}^{3}$. As $\Gamma$ acts properly discontinuously on $\mathbb{H}^{3}, \Lambda(\Gamma) \subset \partial\left(\mathbb{H}^{3}\right)$. Generalizing the work of Patterson [26] for $n=2$, Sullivan 34] constructed a $\Gamma$-invariant conformal density $\left\{\nu_{x}: x \in \mathbb{H}^{3}\right\}$ of dimension $\delta$ supported on $\Lambda(\Gamma)$. Fixing $o \in \mathbb{H}^{3}$, each $\nu_{x}$ is the unique weak limit as $s \rightarrow \delta^{+}$of the family of measures on the compact space $\overline{\mathbb{H}}^{3}:=\mathbb{H}^{3} \cup \partial_{\infty}\left(\mathbb{H}^{3}\right)$ :

$$
\nu_{x, s}:=\frac{1}{\sum_{\gamma \in \Gamma} e^{-s d(o, \gamma(o))}} \sum_{\gamma \in \Gamma} e^{-s d(x, \gamma(o))} \delta_{\gamma(o)}
$$

where $\delta_{\gamma(o)}$ is the dirac measure at $\gamma(o)$. This family will be referred to as the PS density. When $\Gamma$ is of divergence type, i.e., $\mathcal{P}_{\Gamma}(\delta)=\infty$, the PS-density is the unique $\Gamma$-invariant conformal density of dimension $\delta$ (up to a constant multiple) and atom-free [31, Cor. 1.8].

We denote by $\left\{m_{x}: x \in \mathbb{H}^{3}\right\}$ a $G$-invariant conformal density on the boundary $\partial\left(\mathbb{H}^{3}\right)$ of dimension 2 , unique up to homothety. In particular, each $m_{x}$ is invariant under the maximal compact subgroup which stabilizes $x$.

Definition 2.3. (1) The measure $m^{\nu, \nu}$ on $\mathrm{T}^{1}\left(\Gamma \backslash \mathbb{H}^{3}\right)$ is called the BowenMargulis-Sullivan measure $m^{\mathrm{BMS}}$ associated with $\left\{\nu_{x}\right\}$ [35]:

$$
m^{\mathrm{BMS}}(u)=e^{\delta \beta_{u^{+}}(o, \pi(u))} e^{\delta \beta_{u^{-}}(o, \pi(u))} d \nu_{o}\left(u^{+}\right) d \nu_{o}\left(u^{-}\right) d t .
$$

(2) The measure $m^{\nu, m}$ on $\mathrm{T}^{1}\left(\Gamma \backslash \mathbb{H}^{3}\right)$ is called the Burger-Roblin measure $m^{\mathrm{BR}}$ associated with $\left\{\nu_{x}\right\}$ and $\left\{m_{x}\right\}([5],[31)$ :

$$
m^{\mathrm{BR}}(u)=e^{2 \beta_{u^{+}}(o, \pi(u))} e^{\delta \beta_{u^{-}}(o, \pi(u))} d m_{o}\left(u^{+}\right) d \nu_{o}\left(u^{-}\right) d t .
$$


We will refer to these measures as the BMS and the BR measures respectively, for short. It is worth mentioning that the Riemannian volume measure, in these coordinates, is $m^{m, m}$.

The quotient $\Gamma \backslash C(\Lambda(\Gamma))$ of the convex hull $C(\Lambda(\Gamma))$ of the limit set modulo $\Gamma$ is called the convex core of $\Gamma$, denoted by $C(\Gamma)$. A discrete subgroup $\Gamma$ of $G$ is called geometrically finite if a unit neighborhood of the convex core $C(\Gamma)$ has finite volume. It is equivalent to saying that $\Gamma \backslash \mathbb{H}^{3}$ admits a finite sided fundamental domain. A geometrically finite group $\Gamma$ is called convex cocompact if one of the following three equivalent conditions hold (cf. [4]):

(1) $C(\Gamma)$ is compact;

(2) $\Gamma \backslash \mathbb{H}^{3}$ admits a finite sided fundamental domain with no cusps;

(3) $\Lambda(\Gamma)$ consists only of radial limit points: $\xi \in \Lambda(\Gamma)$ is radial if any geodesic ray $\xi_{t}$ toward $\xi$ returns to a compact subset for an unbounded sequence of $t$.

The BMS measure is invariant under the geodesic flow. Sullivan showed that for $\Gamma$ geometrically finite, it is ergodic and moreover the unique measure of maximal entropy (35, 25]). For $\Gamma$ convex cocompact, the support of the BMS measure is compact, as its projection is contained in $C(\Gamma)$.

Theorem 2.4. 9] If $\Gamma$ is geometrically finite and Zariski dense, the PS density of any proper Zariski subvariety of $\partial\left(\mathbb{H}^{3}\right)$ is zero.

2.3. BMS and BR measures on $X=\Gamma \backslash G$. We fix a point $o \in \mathbb{H}^{3}$ whose stabilizer group is $K:=\operatorname{PSU}(2)$. Then the map $g \mapsto g(o)$ induces a $G$-equivariant isometry between $G / K$ and $\mathbb{H}^{3}$. Set

$$
M:=\left\{m_{\theta}=\operatorname{diag}\left(e^{i \theta}, e^{-i \theta}\right)\right\} .
$$

By choosing the unit tangent vector $X_{0}$ based at $o$ stabilized by $M, G / M$ can be identified with the unit tangent bundle $\mathrm{T}^{1}\left(\mathbb{H}^{3}\right)$ via the orbit map $g \mapsto g\left(X_{0}\right)$. This identification can also be lifted to the identification of the frame bundle of $\mathbb{H}^{3}$ with $G$. These identifications are all $\Gamma$-equivariant and induce identifications of the frame bundle of the manifold $\Gamma \backslash \mathbb{H}^{3}$ with $\Gamma \backslash G$. We set $X=\Gamma \backslash G$. Abusing the notation, we will denote by $m^{\mathrm{BMS}}$ and $m^{\mathrm{BR}}$, respectively, the $M$-invariant lifts of the BMS and the BR measures to $X$. For $g \in G$, we set $g^{ \pm}=(g M)^{ \pm}$where $g M \in G / M=\mathrm{T}^{1}\left(\mathbb{H}^{3}\right)$.

For $x=\Gamma \backslash \Gamma g$, we write $x^{ \pm} \in \Lambda(\Gamma)$ if $g^{ \pm} \in \Lambda(\Gamma)$; this is well-defined independent of the choice of $g$. With this notation, the supports of $m^{\mathrm{BMS}}$ and $m^{\mathrm{BR}}$ are given respectively by

$$
\Omega:=\left\{x \in X: x^{+}, x^{-} \in \Lambda(\Gamma)\right\}
$$

and

$$
\Omega_{\mathrm{BR}}:=\left\{x \in X: x^{-} \in \Lambda(\Gamma)\right\} .
$$

The right translation action of the diagonal subgroup

$$
A:=\left\{a_{s}:=\operatorname{diag}\left(e^{s / 2}, e^{-s / 2}\right): s \in \mathbb{R}\right\}
$$

on $G$ is called the frame flow and it projection to $G / M$ corresponds to the geodesic flow. For this action, $m^{\mathrm{BMS}}$ is $A$-invariant and $m^{\mathrm{BR}}$ is $A$-quasi-invariant: $\left(a_{-s}\right)_{*} m^{\mathrm{BR}}=e^{(2-\delta) s} m^{\mathrm{BR}}$. 
Set

$$
N:=\left\{n_{z}=\left(\begin{array}{ll}
1 & 0 \\
z & 1
\end{array}\right): z \in \mathbb{C}\right\} \quad \text { and } \quad \check{N}:=\left\{\check{n}_{z}=\left(\begin{array}{ll}
1 & z \\
0 & 1
\end{array}\right): z \in \mathbb{C}\right\}
$$

and for $g \in G$,

$$
H(g):=g N \quad \text { and } \quad \check{H}(g):=g \check{N} .
$$

The restriction of the projection $G \rightarrow G / M$ induces a diffeomorphism from $H(g)$ (resp. $\check{H}(g))$ to the horosphere $\mathcal{H}_{g M}$ (resp. $\check{\mathcal{H}}_{g M}$ ) in $\mathrm{T}^{1}\left(\mathbb{H}^{3}\right)$ and hence the visual maps $u \rightarrow u^{ \pm}$induce diffeomorphisms $P_{H(g)}: \partial\left(\mathbb{H}^{3}\right)-\left\{g^{-}\right\} \rightarrow H(g)$ and $P_{\breve{H}(g)}: \partial\left(\mathbb{H}^{3}\right)-\left\{g^{+}\right\} \rightarrow \check{H}(g)$, respectively, for each $g \in G$.

Definition 2.5. Let $y \in G$.

(1) Set

$$
d \mu_{H(y)}^{\mathrm{Leb}}(v)=e^{2 \beta_{v^{+}}(o, \pi(v M))} d m_{o}\left(v^{+}\right) \text {for } v \in H(y) .
$$

The measure $\mu_{H(y)}^{\mathrm{Leb}}$ is $G$-invariant: $g_{*} \mu_{H(y)}^{\mathrm{Leb}}=\mu_{g(H(y))}^{\mathrm{Leb}}$; in particular, it is an $N$-invariant measure on $H(y)$.

(2) Set

$$
d \mu_{H(y)}^{\mathrm{PS}}(v)=e^{\delta \beta_{v}+(o, \pi(v M))} d \nu_{o}\left(v^{+}\right) .
$$

We note that $\left\{\mu_{H(y)}^{\mathrm{PS}}\right\}$ is a $\Gamma$-invariant family.

Fix a left $G$-invariant and right $K$-invariant metric on $G$ which induces the hyperbolic distance $d$ on $G / K$.

Notation 2.6. (1) For $\rho>0$ and a subset $Y$ of $G$, we denote by $Y_{\rho}$ the intersection of $Y$ and the $\rho$-ball centered at $e$ in $G$.

(2) The $M$-injectivity radius $\rho_{x}$ at $x \in X$ is the supremum of $\rho$ such that for $B_{\rho}:=\check{N}_{\rho} A_{\rho} M N_{\rho}$, the map $B_{\rho} \rightarrow x_{0} B_{\rho}$ given by $g \rightarrow x_{0} g$ is injective.

Definition 2.7. A box in $X$ (around $x_{0}$ ) refers to a subset of the form

$$
x_{0} B_{\rho}=x_{0} \check{N}_{\rho} A_{\rho} M N_{\rho}
$$

with $0<\rho<\rho_{x_{0}}$ for some $x_{0} \in X$. Note that $x_{0} B_{\rho}$ coincides with $x_{0} \check{N}_{\rho} A_{\rho} N_{\rho} M$. We call this box a BMS box if $x_{0}^{ \pm} \in \Lambda(\Gamma)$, i.e., if $x_{0}$ belongs to the support of the BMS measure.

We fix a box $x_{0} B_{\rho}$. Set $\tilde{T}_{\rho}:=\check{N}_{\rho} A_{\rho}$ and $T_{\rho}:=\check{N}_{\rho} A_{\rho} M$. Since the measures $m^{\mathrm{BMS}}$ and $m^{\mathrm{BR}}$ have the same transverse measures for the unstable horospherical foliations, we have for any $\psi \in C\left(x_{0} B_{\rho}\right)$,

$$
\begin{aligned}
m^{\mathrm{BMS}}(\psi) & =\int_{y \in x_{0} \tilde{T}_{\rho}, m \in M} \int_{n \in N_{\rho}} \psi(y n m) d \mu_{H(y)}^{\mathrm{PS}}(y n) d \tilde{\nu}_{x_{0} \tilde{T}_{\rho}}(y) d m \\
& =\int_{y m \in x_{0} \tilde{T}_{\rho} M} \int_{n \in N_{\rho}} \psi(y m n) d \mu_{H(y m)}^{\mathrm{PS}}(y m n) d\left(\tilde{\nu}_{x_{0} \tilde{T}_{\rho}} \otimes m\right)(y m) \\
m^{\mathrm{BR}}(\psi) & =\int_{y \in x_{0} \tilde{T}_{\rho}, m \in M} \int_{N_{\rho}} \psi(y n m) d \mu_{H(y)}^{\mathrm{Leb}}(y n) d \tilde{\nu}_{x_{0} \tilde{T}_{\rho}}(y) d m \\
& =\int_{y m \in x_{0} \tilde{T}_{\rho} M} \int_{n \in N_{\rho}} \psi(y m n) d \mu_{H(y m)}^{\mathrm{Leb}}(y m n) d\left(\tilde{\nu}_{x_{0} \tilde{T}_{\rho}} \otimes m\right)(y m)
\end{aligned}
$$

that is, $d \nu_{x_{0} T_{\rho}}:=d \tilde{\nu}_{x_{0} \tilde{T}_{\rho}} \otimes d m$ denotes the transverse measure of $m^{\mathrm{BMS}}$ (and hence of $m^{\mathrm{BR}}$ ) on $x_{0} T_{\rho}$. 
The following easily follows from Theorem 2.4

Corollary 2.8. If $\Gamma$ is geometrically finite and Zariski dense, and $E$ is a box in $X$, then $m^{\mathrm{BR}}(\partial(E))=0$.

2.4. BR measure in the Iwasawa coordinates $G=K A N$. The canonical map $\iota: \check{N} \rightarrow G / M A N=K / M$ has a diffeomorphic image $S:=\iota(\check{N})$ which is $K / M$ minus a single point. By abuse of notation, we use the same notation $\nu_{o}$ for the measure on $K$ which is the trivial extension of the PS measure $\nu_{o}$ on $\mathbb{S}^{2}=K / M$ : for $\psi \in C(K)$,

$$
\int_{K} \psi d \nu_{o}=\int_{M} \int_{S} \psi(s m) d \nu_{o}\left(s X_{0}^{-}\right) d m
$$

where $d m$ is the probability Haar measure of $M$. The lift of the BR measure $\tilde{m}^{\text {BR }}$ on $G$ can also be written as follows (cf. [24]): for $\psi \in C_{c}(G)$,

$$
\tilde{m}^{\mathrm{BR}}(\psi)=\int_{G} \psi\left(k a_{s} n_{z}\right) e^{-\delta s} d \nu_{o}(k) d s d z
$$

where $k a_{s} n_{z} \in K A N, d s$ and $d z$ are some fixed Lebesgue measures on $\mathbb{R}$ and $\mathbb{C}$ respectively. As usual, this means that for $\Psi(\Gamma g)=\sum_{\gamma \in \Gamma} \psi(\gamma g)$ with $\psi \in C_{c}(G)$, $m^{\mathrm{BR}}(\Psi)=\tilde{m}^{\mathrm{BR}}(\psi)$.

2.5. BR measure associated to a general unipotent subgroup. A horospherical subgroup $N_{0}$ is a maximal unipotent subgroup of $G$, or equivalently, $N_{0}=\left\{g \in G: b^{n} g b^{-n} \rightarrow e\right.$ as $\left.n \rightarrow \infty\right\}$ for a non-trivial diagonalizable element $b \in G$. Since $A$ normalizes $N$, it follows from the Iwasawa decomposition $G=K A N$ that any horospherical subgroup $N_{0}$ is of the form $k_{0}^{-1} N k_{0}$ for some $k_{0} \in K$. The BR measure associated to $N_{0}$ is defined to be

$$
m_{N_{0}}^{\mathrm{BR}}(\psi):=m^{\mathrm{BR}}\left(k_{0} \cdot \psi\right)
$$

where $\psi \in C_{c}(X)$ and $k_{0} \cdot \psi(g)=\psi\left(g k_{0}\right)$. As $m^{\mathrm{BR}}$ is $M=N_{K}(U)$-invariant (here $N_{K}(U)$ being the normalizer of $U$ in $\left.K\right)$, this definition does not depend on the choice of $k_{0} \in K$. If $U_{0}$ is a one-parameter unipotent subgroup of $G$, its centralizer $C_{G}\left(U_{0}\right)$ in $G$ is a horospherical subgroup. The BR measure associated to $U_{0}$ means $m_{N_{0}}^{\mathrm{BR}}$ for $N_{0}=C_{G}\left(U_{0}\right)$.

2.6. Mixing of frame flow and its consequences. Some of important dynamical properties of flows on $X$ have been established only under the finiteness assumption of the BMS measure. Examples of groups with finite BMS measure include all geometrically finite groups [34] but not limited to those (see [27]). Roblin showed that if $\left|m^{\mathrm{BMS}}\right|<\infty$, then $\Gamma$ is of divergence type. In the following two theorems, we consider the groups $\Gamma$ with $\left|m^{\mathrm{BMS}}\right|<\infty$. We normalize $\nu_{o}$ so that $\left|m^{\mathrm{BMS}}\right|=1$.

The following two theorems were proved by [36], based on the the previous works of Babillot [2], Roblin [31], and Flaminio-Spatzier [9].

Theorem 2.9. 36] Suppose that $\Gamma$ is Zariski dense and $\left|m^{\mathrm{BMS}}\right|=1$.

(1) The frame flow on $X$ is mixing with respect to $m^{\mathrm{BMS}}$, that is, for any $\psi_{1}, \psi_{2} \in L^{2}\left(X, m^{\mathrm{BMS}}\right)$, as $s \rightarrow \pm \infty$,

$$
\int_{X} \psi_{1}\left(x a_{s}\right) \psi_{2}(x) d m^{\mathrm{BMS}}(x) \rightarrow m^{\mathrm{BMS}}\left(\psi_{1}\right) m^{\mathrm{BMS}}\left(\psi_{2}\right) .
$$

(2) The BR measure $m^{\mathrm{BR}}$ on $X$ is $N$-ergodic. 
(3) If $\Gamma$ is geometrically finite, $m^{\mathrm{BR}}$ is the only $N$-ergodic measure on $X$ which is not supported on a closed $N$-orbit.

Theorem 2.10. 36] Let $\Gamma$ be Zariski dense and $\left|m^{\mathrm{BMS}}\right|=1$. Then for all $\psi_{1}, \psi_{2} \in$ $C_{c}(X)$ or for $\psi_{1}=\chi_{E_{1}}, \psi_{2}=\chi_{E_{2}}$ where $E_{i} \subset X$ is a bounded Borel subset with $m^{\mathrm{BMS}}\left(\partial\left(E_{i}\right)\right)=0$, we have: as $s \rightarrow+\infty$,

$$
\int_{X} \psi_{1}\left(x a_{-s}\right) \psi_{2}(x) d m^{\mathrm{BR}}(x) \rightarrow m^{\mathrm{BMS}}\left(\psi_{1}\right) m^{\mathrm{BR}}\left(\psi_{2}\right) .
$$

We note that by the quasi-invariance of the BR measure,

$$
\int_{X} \psi_{1}\left(x a_{-s}\right) \psi_{2}(x) d m^{\mathrm{BR}}(x)=e^{(2-\delta) s} \int_{X} \psi_{1}(x) \psi_{2}\left(x a_{s}\right) d m^{\mathrm{BR}}(x) .
$$

In particular, the above theorem implies that if $\delta<2$,

$$
\int_{X} \psi_{1}(x) \psi_{2}\left(x a_{s}\right) d m^{\mathrm{BR}}(x) \rightarrow 0 \quad \text { as } s \rightarrow+\infty .
$$

Lemma 2.11. If $\Gamma$ is a discrete subgroup of $G$ with $\delta>1$, then $\Gamma$ is Zariski dense in $G$.

Proof. Let $G_{0}$ be the identity component of the Zariski closure of $\Gamma$. Suppose $G_{0}$ is a proper subgroup of $G$. Being an algebraic subgroup of $G, G_{0}$ is contained either in a parabolic subgroup of $G$ or in a subgroup isomorphic to $\operatorname{PSL}_{2}(\mathbb{R})$. In either case, the critical exponent of $G_{0}$ is at most 1 . This leads to a contradiction and hence $G_{0}=G$.

\section{WEAK CONVERGENCE OF THE CONDITIONAL OF $\mu_{E, s}^{\mathrm{BR}}$}

In this section, we suppose that $\Gamma$ is a Zariski dense discrete subgroup of $G$ admitting a finite BMS measure, which we normalize so that $\left|m^{\mathrm{BMS}}\right|=1$.

Fix a bounded $M$-invariant Borel subset $E \subset X$ with $m^{\mathrm{BR}}(E)>0$ and $m^{\mathrm{BR}}(\partial(E))=0$.

For each $s>0$, define a Borel measure $\mu_{E, s}^{\mathrm{BR}}$ on $X$ to be the normalization of the push-forward $\left.\left(a_{-s}\right) * m^{\mathrm{BR}}\right|_{E}$ : for $\Psi \in C_{c}(X)$,

$$
\mu_{E, s}^{\mathrm{BR}}(\Psi):=\frac{1}{m^{\mathrm{BR}}(E)} \int_{E} \Psi\left(x a_{-s}\right) d m^{\mathrm{BR}}(x) .
$$

Equivalently,

$$
\mu_{E, s}^{\mathrm{BR}}(\Psi)=\frac{e^{(2-\delta) s}}{m^{\mathrm{BR}}(E)} \int_{X} \Psi(x) \chi_{E}\left(x a_{s}\right) d m^{\mathrm{BR}}(x) .
$$

Note that $\mu_{E, s}^{\mathrm{BR}}$ is a probability measure supported in the set $E a_{-s}$.

The following is immediate from Theorem 2.10.

Theorem 3.1. As $s \rightarrow+\infty, \mu_{E, s}^{\mathrm{BR}}$ weakly converges to $m^{\mathrm{BMS}}$, that is, for any $\Psi \in C_{c}(X)$,

$$
\lim _{s \rightarrow+\infty} \mu_{E, s}^{\mathrm{BR}}(\Psi)=m^{\mathrm{BMS}}(\Psi) .
$$

For simplicity, we will write for $x \in X$,

$$
d \lambda_{x}(n)=d \mu_{H(x)}^{\mathrm{Leb}}(x n) \text { and } d \mu_{x}^{\mathrm{PS}}(n)=d \mu_{H(x)}^{\mathrm{PS}}(x n)
$$

so that $\lambda_{x}$ and $\mu_{x}^{\mathrm{PS}}$ are respectively the conditional measures of $m^{\mathrm{BR}}$ and $m^{\mathrm{BMS}}$ on $x N$. 
Recall that $\rho_{x}$ denotes the injectivity radius at $x$.

Definition 3.2. Fix $x \in X$. For $s>0$, define a Borel measure $\lambda_{E, x, s}$ on $x N_{\rho_{x}}$ as follows: for $\psi \in C_{c}\left(x N_{\rho_{x}}\right)$,

$$
\lambda_{E, x, s}(\psi):=\frac{e^{(2-\delta) s}}{m^{\mathrm{BR}}(E)} \int_{n \in N_{\rho_{x}}} \psi(x n) \chi_{E}\left(x n a_{s}\right) d \lambda_{x}(n) .
$$

Recall the notation $T_{\rho}=\check{N}_{\rho} A_{\rho} M$ for $\rho>0$. Let $x_{0} \in X$ and let $0<\rho \leq \rho_{x_{0}}$. For any box $x_{0} B_{\rho}=x_{0} T_{\rho} N_{\rho}$ and $\Psi \in C\left(x_{0} B_{\rho}\right)$, we have

$$
\begin{aligned}
\mu_{E, s}^{\mathrm{BR}}(\Psi) & =\frac{e^{(2-\delta) s}}{m^{\mathrm{BR}}(E)} \int_{x \in X} \Psi(x) \chi_{E}\left(x a_{s}\right) d m^{\mathrm{BR}}(x) \\
& =\frac{e^{(2-\delta) s}}{m^{\mathrm{BR}}(E)} \int_{x \in x_{0} T_{\rho}} \int_{n \in N_{\rho}} \Psi(x n) \chi_{E}\left(x n a_{s}\right) d \lambda_{x}(n) d \nu_{x_{0} T_{\rho}}(x) \\
& =\int_{x \in x_{0} T_{\rho}} \lambda_{E, x, s}\left(\left.\Psi\right|_{x N_{\rho}}\right) d \nu_{x_{0} T_{\rho}}(x) .
\end{aligned}
$$

Hence $\lambda_{E, x, s}$ is precisely the conditional measure of $\mu_{E, s}^{\mathrm{BR}}$ on $x N_{\rho}$.

The aim of this section is to prove:

Theorem 3.3. Suppose that $x^{-} \in \Lambda(\Gamma)$ and $0<\rho<\rho_{x}$. For any $\psi \in C_{c}\left(x N_{\rho}\right)$,

$$
\lambda_{E, x, s}(\psi) \longrightarrow \mu_{x}^{\mathrm{PS}}(\psi) \text { as } s \rightarrow+\infty .
$$

The condition $x^{-} \in \Lambda(\Gamma)$ is needed to approximate the measure $\lambda_{E, x, s}$ by its thickening in the transverse direction.

For a function $\Psi$ on $X$ and $\varepsilon>0$, we define functions on $X$ as follows:

$$
\Psi_{\varepsilon}^{+}(y):=\sup _{g \in \mathcal{O}_{\varepsilon}} \Psi(y g) \text { and } \Psi_{\varepsilon}^{-}(y):=\inf _{g \in \mathcal{O}_{\varepsilon}} \Psi(y g)
$$

where $\mathcal{O}_{\varepsilon}$ is a symmetric $\varepsilon$-neighborhood of $e$ in $G$. We also set

$$
E_{\varepsilon}^{+}:=E \mathcal{O}_{\varepsilon} \quad \text { and } \quad E_{\varepsilon}^{-}=\cap_{u \in \mathcal{O}_{\varepsilon}} E u .
$$

Lemma 3.4. Let $x \in X$ and $0<\rho<\rho_{x}$. For all small $\varepsilon>0$, there exists $\varepsilon_{1}>0$ such that for any non-negative $\Psi \in C\left(x T_{\varepsilon_{1}} N_{\rho}\right)$ and any $t \in T_{\varepsilon_{1}}$, we have

$$
e^{-\varepsilon} \lambda_{x}\left(\Psi_{\varepsilon}^{-}\right) \leq \lambda_{x t}(\Psi) \leq e^{\varepsilon} \lambda_{x}\left(\Psi_{\varepsilon}^{+}\right) .
$$

Proof. Let $0<\varepsilon<\rho_{x}-\rho$. Consider the map $\phi_{t}: x N \rightarrow x t N$ given by $\phi_{t}(x n)=x t n$, so that $\phi_{t}^{*} \lambda_{x t}=\lambda_{x}$. Since $\phi_{t}$ is a translation by $n^{-1} t n$, there exists $\varepsilon_{1}>0$ such that for all $n \in N_{\rho}$ and $t \in T_{\varepsilon_{1}}, n^{-1} t n \in \mathcal{O}_{\varepsilon}$ and the Radon-Nikodym derivative satisfies $e^{-\varepsilon} \leq \frac{d \lambda_{x t}}{d \lambda_{x}}(n) \leq e^{\varepsilon}$.

Therefore

$$
\lambda_{x t}(\Psi)=\int \Psi\left(x n\left(n^{-1} t n\right)\right) d \lambda_{x t}(n) \leq e^{\varepsilon} \int \Psi_{\varepsilon}^{+}(x n) d \lambda_{x}(n)=e^{\varepsilon} \lambda_{x}\left(\Psi_{\varepsilon}^{+}\right) .
$$

The other inequality follows similarly.

Lemma 3.5. Let $x \in X$ and $0<\rho<\rho_{x}$. For any $\varepsilon>0$, there exists $\varepsilon_{1}>0$ such that for any non-negative $\Psi \in C\left(x T_{\varepsilon_{1}} N_{\rho}\right)$, any $t \in T_{\varepsilon_{1}}$ and any $s>0$,

$$
e^{-\varepsilon} \lambda_{E_{\varepsilon}^{-}, x, s}\left(\Psi_{2 \varepsilon}^{-}\right) \leq \lambda_{E, x t, s}(\Psi) \leq e^{\varepsilon} \lambda_{E_{\varepsilon}^{+}, x, s}\left(\Psi_{2 \varepsilon}^{+}\right) .
$$


Proof. Let $\varepsilon_{1}>0$ be as in Lemma 3.4. We may also assume that $n \mathcal{O}_{\varepsilon_{1}} n^{-1} \subset \mathcal{O}_{\varepsilon}$ for all $n \in N_{\rho}$.

For $t=\left(\begin{array}{cc}\alpha & w \\ 0 & \alpha^{-1}\end{array}\right) \in \check{N} A M$ and $n_{z} \in N$ with $\alpha+z w \neq 0$, define

$$
\psi_{t}(z)=\frac{z^{2} w+\alpha z-\alpha^{-1} z}{\alpha+z w} \quad \text { and } \quad b_{t, z}=\left(\begin{array}{cc}
\alpha+z w & w \\
0 & \psi_{t}(z) w+\alpha^{-1}-z w
\end{array}\right) .
$$

Then by a direct computation, we verify that

$$
t n_{z}=n_{z+\psi_{t}(z)} b_{t, z}
$$

Therefore we may assume that $\varepsilon_{1}>0$ is small enough so that for all $t \in T_{\varepsilon_{1}}$ and $n_{z} \in N_{\rho}$, we have $\left\{n_{\psi_{t}(z)}: n_{z} \in N_{\rho}\right\} \subset N_{\varepsilon}, b_{t, z} \in T_{\varepsilon}$, and the absolute value of the Jacobian of the map $\left.\psi_{t}\right|_{N_{\rho}}$ is at most $\varepsilon / 2$.

We observe that $n_{z} a_{s}=n_{z+\psi_{t}(z)} a_{s}\left(a_{-s} b_{t, z} a_{s}\right)$ and since the conjugation by $a_{-s}$ contracts $\check{N} A$ for $s>0$, we have $n_{z} a_{s} \in n_{z+\psi_{t}(z)} a_{s} \mathcal{O}_{\varepsilon} M$.

Since $E$ is $M$-invariant, we deduce that $\chi_{E}\left(x t n_{z} a_{s}\right) \leq \chi_{E_{\varepsilon}^{+}}\left(x n_{z+\psi_{t}(z)} a_{s}\right)$ for all $t \in T_{\varepsilon_{1}}$ and $n_{z} \in N_{\rho}$. Together with Lemma 3.4. we now obtain that for any $t \in T_{\varepsilon_{1}}$,

$$
\begin{aligned}
\lambda_{E, x t, s}(\Psi) & =e^{(2-\delta) s} \int_{n_{z} \in N_{\rho}} \Psi\left(x t n_{z}\right) \chi_{E}\left(x t n_{z} a_{s}\right) d \lambda_{x t}(z) \\
& \leq e^{(2-\delta) s} \int_{n_{z} \in N_{\rho}} \Psi_{\varepsilon}^{+}\left(x n_{z}\right) \chi_{E_{\varepsilon}^{+}}\left(x n_{z+\psi_{t}(z)} a_{s}\right) d \lambda_{x t}(z) \\
& \leq e^{\varepsilon} e^{(2-\delta) s} \int_{n_{z} \in N_{\rho}} \Psi_{\varepsilon}^{+}\left(x n_{z}\right) \chi_{E_{\varepsilon}^{+}}\left(x n_{z+\psi_{t}(z)} a_{s}\right) d \lambda_{x}(z) \\
& \leq e^{2 \varepsilon} e^{(2-\delta) s} \int_{n_{z} \in N_{\rho+\varepsilon}} \Psi_{\varepsilon}^{+}\left(x n_{z-\psi_{t}(z)}\right) \chi_{E_{\varepsilon}^{+}}\left(x n_{z} a_{s}\right) d \lambda_{x}(z) \\
& \leq e^{2 \varepsilon} e^{(2-\delta) s} \int_{n_{z} \in N_{\rho+3 \varepsilon}} \Psi_{2 \varepsilon}^{+}\left(x n_{z}\right) \chi_{E_{\varepsilon}^{+}}\left(x n_{z} a_{s}\right) d \lambda_{x}(z) \\
& =e^{2 \varepsilon} \lambda_{E_{\varepsilon}^{+}, x, s}\left(\Psi_{2 \varepsilon}^{+}\right)
\end{aligned}
$$

where the last inequality follows since $N_{\rho+3 \varepsilon}$ contains $x N \cap \operatorname{supp}\left(\Psi_{2 \varepsilon}^{+}\right)$. The other inequality can be proven similarly.

Theorem 3.3 follows from:

Theorem 3.6. Let $x^{-} \in \Lambda(\Gamma)$ and $\rho<\rho_{x}$. Let $\psi \in C\left(x N_{\rho}\right)$ be a non-negative function. For $\varepsilon>0$, there exists $s_{0} \gg 1$ such that for any $s>s_{0}$,

$$
e^{-4 \varepsilon} \lambda_{E, x, s}(\psi) \leq \mu_{x}^{\mathrm{PS}}(\psi) \leq e^{4 \varepsilon} \lambda_{E, x, s}(\psi) .
$$

Moreover, if $x^{+} \in \Lambda(\Gamma)$ and $\psi$ is positive, then the above integrals are all non-zero.

Proof. Let $\varepsilon_{1}$ be as in Lemma 3.5. We note that as $x^{-} \in \Lambda(\Gamma), \nu\left(x T_{\varepsilon_{1}}\right)>0$. Hence there exists a non-negative continuous function $\phi \in C\left(x T_{\varepsilon_{1}}\right)$ with $\nu(\phi)=1$. Define $\Psi \in C\left(x T_{\varepsilon_{1}} N_{\rho}\right)$ by

$$
\Psi(x t n):=\psi(x n) \phi(x t) \quad \text { for } x t n \in x T_{\varepsilon_{1}} N_{\rho} .
$$


Set $\psi_{\varepsilon}^{+}(x n)=\sup _{u \in N_{\varepsilon}} \psi(x n u)$ and $\psi_{\varepsilon}^{-}(x n)=\inf _{u \in N_{\varepsilon}} \psi(x n u)$. Then by Lemma 3.5

$$
\begin{aligned}
\mu_{E, s}^{\mathrm{BR}}(\Psi) & =\int_{x T_{\varepsilon_{1}}} \lambda_{E, x t, s}(\Psi) d \nu_{x T_{\rho}}(x t) \\
& \leq e^{\varepsilon} \int_{x T_{\varepsilon_{1}}} \lambda_{E_{\varepsilon}^{+}, x, s}\left(\psi_{2 \varepsilon}^{+}\right) \phi(x t) d \nu_{x T_{\rho}}(x t) \\
& =e^{\varepsilon} \lambda_{E_{\varepsilon}^{+}, x, s}\left(\psi_{2 \varepsilon}^{+}\right) .
\end{aligned}
$$

We can prove the other inequality similarly and hence

$$
e^{-\varepsilon} \lambda_{E_{\varepsilon}^{-}, x, s}\left(\psi_{2 \varepsilon}^{-}\right) \leq \mu_{E, s}^{\mathrm{BR}}(\Psi) \leq e^{\varepsilon} \lambda_{E_{\varepsilon}^{+}, x, s}\left(\psi_{2 \varepsilon}^{+}\right) .
$$

Since the map $t \mapsto \mu_{x t}^{\mathrm{PS}}$ is continuous by [31, Lemma 1.16], we have

$$
e^{-\varepsilon} \mu_{x}^{\mathrm{PS}}(\psi) \leq m^{\mathrm{BMS}}(\Psi) \leq e^{\varepsilon} \mu_{x}^{\mathrm{PS}}(\psi)
$$

by replacing $\varepsilon_{1}$ by a smaller one if necessary.

Since $\mu_{E, s}^{\mathrm{BR}}(\Psi) \rightarrow m^{\mathrm{BMS}}(\Psi)$ by Theorem 3.1, we deduce from (3.2) that there exists $s_{0}>1$ such that for all $s>s_{0}$,

$$
e^{-2 \varepsilon} \lambda_{E_{\varepsilon}^{-}, x, s}\left(\psi_{2 \varepsilon}^{-}\right) \leq \mu_{x}^{\mathrm{PS}}(\psi) \leq e^{2 \varepsilon} \lambda_{E_{\varepsilon}^{+}, x, s}\left(\psi_{2 \varepsilon}^{+}\right) .
$$

We claim that

$$
e^{-4 \varepsilon} \lambda_{E, x, s}(\psi) \leq \lambda_{E_{\varepsilon}^{ \pm}, x, s}\left(\psi_{2 \varepsilon}^{ \pm}\right) \leq e^{4 \varepsilon} \lambda_{E, x, s}(\psi)
$$

which will complete the proof of the theorem by (3.3).

We can deduce from (3.2) that

$$
e^{-\varepsilon} \mu_{E_{2 \varepsilon}^{-}, s}^{\mathrm{BR}}\left(\Psi_{4 \varepsilon}^{-}\right) \leq \lambda_{E_{\varepsilon}^{-}, x, s}\left(\psi_{2 \varepsilon}^{-}\right) \leq \lambda_{E_{\varepsilon}^{+}, x, s}\left(\psi_{2 \varepsilon}^{+}\right) \leq e^{\varepsilon} \mu_{E_{2 \varepsilon}^{+}, s}^{\mathrm{BR}}\left(\Psi_{4 \varepsilon}^{+}\right) .
$$

Since it follows from Theorem 3.1 that

$$
e^{-\varepsilon} \mu_{E, s}^{\mathrm{BR}}(\Psi) \leq \mu_{E_{2 \varepsilon}^{ \pm}, s}^{\mathrm{BR}}\left(\Psi_{4 \varepsilon}^{ \pm}\right) \leq e^{\varepsilon} \mu_{E, s}^{\mathrm{BR}}(\Psi) \text { for all large } s \gg 1,
$$

we have

$$
e^{-2 \varepsilon} \mu_{E, s}^{\mathrm{BR}}(\Psi) \leq \lambda_{E_{\varepsilon}^{-}, x, s}\left(\psi_{2 \varepsilon}^{-}\right) \leq \lambda_{E_{\varepsilon}^{+}, x, s}\left(\psi_{2 \varepsilon}^{+}\right) \leq e^{2 \varepsilon} \mu_{E, s}^{\mathrm{BR}}(\Psi) .
$$

This implies

$$
\lambda_{E_{\varepsilon}^{+}, x, s}\left(\psi_{2 \varepsilon}^{+}\right) \leq e^{4 \varepsilon} \lambda_{E_{\varepsilon}^{-}, x, s}\left(\psi_{2 \varepsilon}^{-}\right)
$$

and hence (3.4) follows.

\section{PS DENSITY AND ITS NON-FOCUSING PROPERTY WHEN $\delta>1$}

Let $\Gamma$ be a (non-elementary) convex cocompact subgroup of $G$.

The assumption on $\Gamma$ being convex cocompact is crucial for the following theorem:

Theorem 4.1. For any compact subset $F_{0}$ of $X$, there exists $c_{0}=c_{0}\left(F_{0}\right)>1$ such that for any $x \in F_{0}$ with $x^{+} \in \Lambda(\Gamma)$ and for all $0<r \ll 1$,

$$
c_{0}^{-1} r^{\delta} \leq \mu_{H(x)}^{\mathrm{PS}}\left(x N_{r}\right) \leq c_{0} r^{\delta}
$$

where $x N_{r}=\left\{x n_{z}:|z|<r\right\}$. 
Similarly, for any $x \in F_{0}$ with $x^{-} \in \Lambda(\Gamma)$ and for all $0<r \ll 1$, we have

$$
c_{0}^{-1} r^{\delta} \leq \mu_{\check{H}(x)}^{\mathrm{PS}}\left(x \check{N}_{r}\right) \leq c_{0} r^{\delta}
$$

for $x \check{N}_{r}=\left\{x \check{n}_{z}:|z|<r\right\}$.

Proof. As $F_{0}$ is compact, up to uniform constants, $\mu_{H(x)}^{\mathrm{PS}}\left(x N_{r}\right) \asymp \nu_{o}\left(B\left(x^{+}, r\right)\right)$ where $B\left(x^{+}, r\right)$ is the ball around $x^{+}$of radius $r$ in $\partial\left(\mathbb{H}^{3}\right)$ in the spherical metric. As $x^{+} \in \Lambda(\Gamma)$, the above result is then due to Sullivan 35] who says $\nu_{o}(B(\xi, r)) \asymp r^{\delta}$ uniformly for all $\xi \in \Lambda(\Gamma)$ and for all small $r>0$ for $\Gamma$ convex cocompact.

Lemma 4.2. Let $\delta>1$ and $F_{0} \subset X$ be a compact subset. For every $\varepsilon>0$, there exists a positive integer $d=d\left(\varepsilon, F_{0}\right)$ such that for any $x \in F_{0}$ with $x^{-} \in \Lambda(\Gamma)$ and for all small $0<r \ll 1$, we have

$$
\mu_{\check{H}(x)}^{\mathrm{PS}}\left\{x \check{n}_{z}:|z|<r,|\Im(z)| \leq \frac{|\Re(z)|}{d}\right\} \leq \varepsilon \cdot \mu_{\check{H}(x)}^{\mathrm{PS}}\left(x \check{N}_{r}\right)
$$

Proof. Let $r$ be small enough to satisfy Theorem 4.1. For an integer $d \geq 1$, consider

$$
\mathcal{B}_{d}(x, r):=\left\{x \check{n}_{z}:|z|<r,|\Im(z)|<r / d\right\}
$$

which clearly contains the set in question. Theorem 4.1 implies that

$$
\mu_{\breve{H}(x)}^{\mathrm{PS}}\left(\mathcal{B}_{d}(x, r)\right) \leq c_{0} \frac{d \cdot r^{\delta}}{d^{\delta}}=c_{0} d^{1-\delta} r^{\delta},
$$

where $c_{0}>1$ is an absolute constant independent of $d$ and $r$.

Let $d=d(\varepsilon) \gg 1$ be such that $c_{0} d^{1-\delta}<c_{0}^{-1} \varepsilon$. Then $\mu_{\tilde{H}(x)}^{\mathrm{PS}}\left(\mathcal{B}_{d}(x, r)\right) \leq \varepsilon$. $\mu_{\check{H}(x)}^{\mathrm{PS}}\left(x \check{N}_{r}\right)$, implying the claim.

Lemma 4.3. There exists $b_{0}>1$ such that for all small $0<\eta \ll 1$

$$
N_{b_{0}^{-1} \eta} A_{b_{0}^{-1} \eta} \check{N}_{b_{0}^{-1} \eta} M \subset \check{N}_{\eta} A_{\eta} N_{\eta} M \subset \check{N}_{b_{0} \eta} A_{b_{0} \eta} N_{b_{0} \eta} M .
$$

Proof. The claim follows since the product maps $\check{N} \times A \times N \times M \rightarrow G$ by $(\check{n}, a, n, m) \mapsto \check{n} a n m$ and $N \times A \times \check{N} \times M \rightarrow G$ by $(n, a, \check{n}, m) \mapsto n a \check{n} m$ are local diffeomorphisms at the identity.

We will use the above results to prove the following proposition 4.4. The proof is elementary and is based on the fact we have a good control of the conditional measures on contracting leaves, i.e., $N$-orbits. However, the fact that this statement holds is quite essential to our approach. Indeed, as we explained in the introduction, one major difficulty we face is that the return times for our $U$-flow do not have the regularity one needs in order to get the required ergodic theorem on the nose. In our version of the window theorem, the set where a window estimate holds depends on time; see Section 7 and in particular Theorem 7.7 below. Usually, in arguments with a similar structure as ours, this fact is fatal as one has very little control on the structure of the "generic" set for the measure in question. In our case, however, the following proposition saves the day and provides us with a rather strong control.

In the following proposition we fix a BMS box $E=x_{0} \check{N}_{\rho} A_{\rho} N_{\rho} M$ with $x_{0}^{ \pm} \in \Lambda(\Gamma)$ and $0<\rho<\frac{1}{b_{0}} \inf _{x \in \Omega} \rho_{x}$ where $b_{0}$ is as in Lemma4.3 and $\rho_{x}$ is the injectivity radius at $x$.

Proposition 4.4. Let $\delta>1$. Fix $0<r<1$. There exists positive numbers $d_{0}=$ $d_{0}(r)>1$ and $s_{0} \gg 1$ such that for any Borel subset $F \subset E$ with $m^{\mathrm{BR}}(F)>$ 
$r \cdot m^{\mathrm{BR}}(E)$ and any $s \geq s_{0}$, there exists a pair of elements $x_{s}, y_{s} \in F$ satisfying

(1) $x_{s}=y_{s} \check{n}_{w_{s}}$ for $\check{n}_{w_{s}} \in \check{N}$,

(2) $\frac{1}{d_{0} s} \leq\left|w_{s}\right| \leq \frac{d_{0}}{s}$ and

(3) $\left|\Im\left(w_{s}\right)\right| \geq \frac{\left|\Re\left(w_{s}\right)\right|}{d_{0}}$.

Proof. Let $c_{0}>1$ be as in Theorem 4.1 where $F_{0}$ is the $2 \rho$-neighborhood of $\Omega$. We will write $B(z, \rho)=z \check{N}_{\rho}$ in this proof. For all $x \in x_{0} N_{\rho} A_{\rho} M, x^{-}=x_{0}^{-}$and hence $x^{-} \in \Lambda(\Gamma)$. Hence by Theorem 4.1

$$
c_{0}^{-1} \eta^{\delta} \leq \mu_{\check{H}(x)}^{\mathrm{PS}}(B(x, \eta)) \leq c_{0} \eta^{\delta} \text { for all } 0<\eta<1 .
$$

Set $d_{1}:=\frac{\nu\left(x_{0} N_{b_{0} \rho} A_{b_{0} \rho} M\right)}{m^{\mathrm{BR}}(E)}$ where $\nu$ denotes the transverse measure of $m^{\mathrm{BR}}$ on $x_{0} N_{\rho_{x_{0}}} A_{\rho_{x_{0}}} M$. We claim that there exists $z \in x_{0} N_{b_{0} \rho} A_{b_{0} \rho} M$ with $\mu_{\breve{H}(z)}^{\mathrm{PS}}\left(B\left(z, b_{0} \rho\right) \cap\right.$ $F)>\frac{r}{d_{1}}$. Suppose not; then

$$
\begin{aligned}
m^{\mathrm{BR}}(F) & \leq \int_{z \in x_{0} N_{b_{0} \rho} A_{b_{0} \rho} M} \mu_{\breve{H}(z)}^{\mathrm{PS}}\left(B\left(z, b_{0} \rho\right) \cap F\right) d \nu(z) \\
& \leq \frac{r}{d_{1}} \nu\left(x_{0} N_{b_{0} \rho} A_{b_{0} \rho} M\right)=r \cdot m^{\mathrm{BR}}(E)
\end{aligned}
$$

which contradicts the assumption on $F$.

Set $Q:=B\left(z, b_{0} \rho\right) \cap F \cap \operatorname{supp}\left(\mu_{\tilde{H}(z)}^{\mathrm{PS}}\right)$ and for each $s>1$, consider the covering $\left\{B\left(x, s^{-1}\right) \subset \check{H}(z): x \in Q\right\}$ of $Q$. By the Besicovitch covering lemma (cf. [20]), there exist $\kappa>0$ (independent of $s$ ) and a finite subset $Q_{s}$ such that the corresponding finite subcover $\left\{B\left(x, s^{-1}\right): x \in Q_{s}\right\}$ of $Q$ is of multiplicity at most $\kappa$.

Note that for $q>1$, by (4.1),

$$
\mu_{\tilde{H}(z)}^{\mathrm{PS}}\left(\cup_{x \in Q_{s}} B\left(x, \frac{1}{q s}\right)\right) \leq \kappa^{2} q^{-\delta} c_{0}^{2} \mu_{\check{H}(z)}^{\mathrm{PS}}\left(\cup_{x \in Q_{s}} B\left(x, \frac{1}{s}\right)\right) \leq \kappa^{2} q^{-\delta} c_{0}^{3} b_{0}^{\delta} \rho^{\delta} .
$$

Hence by taking $q \geq 1$ large so that $\kappa^{2} q^{-\delta} c_{0}^{3} b_{0}^{\delta} \rho^{\delta}<\frac{r}{3 d_{1}}$, we have

$$
\mu_{\tilde{H}(z)}^{\mathrm{PS}}\left(\cup_{x \in Q_{s}} B\left(x, \frac{1}{q s}\right)\right)<\frac{r}{3 d_{1}} .
$$

If we set

$$
R(s, d):=\cup_{x \in Q_{s}}\left\{w \in B\left(x, \frac{1}{s}\right):|\Im(w)| \leq \frac{|\Re(w)|}{d}\right\},
$$

it follows from Lemma 4.2 that there exist $d_{2}>1$ and $s_{0}>1$ such that for any $s>s_{0}$,

$$
\mu_{\tilde{H}(z)}^{\mathrm{PS}}\left(R\left(s, d_{2}\right)\right)<\frac{r}{3 d_{1}} .
$$

Hence for any $s>s_{0}$, the set

$$
Q-\left(\cup_{x \in Q_{s}} B\left(x, \frac{1}{q s}\right) \cup R\left(s, d_{2}\right)\right)
$$

has a positive $\mu_{\breve{H}(z)}^{\mathrm{PS}}$ measure (at least $\frac{r}{3 d_{1}}$ ). In particular, there exists $x_{s} \in Q$ such that $\left(Q \cap B\left(x_{s}, \frac{1}{s}\right)\right)-\left(B\left(x_{s}, \frac{1}{q s}\right) \cup R\left(s, d_{2}\right)\right)$ has a positive $\mu_{\breve{H}(z)}^{\mathrm{PS}}$ measure. Picking $y_{s}$ from this set, we have found a desired pair $x_{s}, y_{s}$ from $F$ with $d_{0}=\max \left(q, d_{1}\right)$. 


\section{EnERGy ESTimate AND $L^{2}$-CONVERGENCE FOR THE PROJECTIONS}

Let $\Gamma$ be a convex cocompact subgroup of $G$ with $\delta>1$ and fix a BMS box $E \subset X$ (see 2.7 for its definition). We have $m^{\mathrm{BR}}(E)>0$ and by Lemma 2.11 and Corollary 2.8, $m^{\mathrm{BR}}(\partial(E))=0$.

In the entire section, we fix $x \in X$ with $x^{ \pm} \in \Lambda(\Gamma)$ and $0<\rho<\frac{1}{\sqrt{2}} \rho_{x}$.

Recall the definition of the measure $\lambda_{E, x, s}$ on $x N_{\rho_{x}}$ from (3.2): for $\psi \in C\left(x N_{\rho_{x}}\right)$,

$$
\lambda_{E, x, s}(\psi)=\frac{e^{(2-\delta) s}}{m^{\mathrm{BR}}(E)} \int_{n \in N_{\rho_{x}}} \psi(x n) \chi_{E}\left(x n a_{s}\right) d \lambda_{x}(n) .
$$

5.1. Projections of $\mu_{H(x)}^{\mathrm{PS}}$ and $\lambda_{E, x, s}$. The $N$-orbit of $x$ can be identified with $\mathbb{R}^{2}$ via the visual map $x n \mapsto(x n)^{+} \in \partial\left(\mathbb{H}^{3}\right)-\left\{x^{-}\right\}$and the identification of $\partial\left(\mathbb{H}^{3}\right)-\left\{x^{-}\right\}$with $\mathbb{R}^{2}$ by mapping $x^{-}$to the point at infinity. Therefore, we may consider $\lambda_{E, x, s}$ and $\mu_{H(x)}^{\mathrm{PS}}$ as measures on $\mathbb{R}^{2}$.

Let

$$
U=\left\{\left(\begin{array}{cc}
1 & 0 \\
t & 1
\end{array}\right): t \in \mathbb{R}\right\} ; \quad V=\left\{\left(\begin{array}{cc}
1 & 0 \\
i t & 1
\end{array}\right): t \in \mathbb{R}\right\} .
$$

In the sequel by a measure on $[0,2 \pi]$ we mean the normalized Lebesgue measure. For each $\theta \in[0,2 \pi)$, we set $U_{\theta}=m_{\theta} U m_{\theta}^{-1}$ and $V_{\theta}=m_{\theta} V m_{\theta}^{-1}$. We may identify $U_{\theta}$ as the line in $\mathbb{R}^{2}$ in the $\theta$-direction and $V_{\theta}$ as the line in the $\theta+\pi / 2$ direction.

We denote by $p_{\theta}: U_{\theta} V_{\theta} \rightarrow V_{\theta}$ the projection parallel to the line $U_{\theta}$. For $\tau>0$, set

$$
U_{\theta}^{\tau}:=\{t \exp (i \theta): t \in[-\tau, \tau]\} \text { and } V_{\theta}^{\tau}:=\{i t \exp (i \theta): t \in[-\tau, \tau]\} .
$$

Definition 5.1. Fix $0<\theta<\pi, 0<\tau \leq \rho$ and $s>1$. We define the measures on $x V_{\theta}^{\tau}$ as follows: for $\psi \in C_{c}\left(x V_{\theta}^{\tau}\right)$,

$$
\sigma_{x, \theta}^{\tau}(\psi):=\int_{x V_{\theta}^{\rho} U_{\theta}^{\tau}} \psi\left(p_{\theta}(y)\right) d \mu_{H(x)}^{\mathrm{PS}}(y),
$$

and

$$
\sigma_{x, \theta, s}^{\tau}(\psi):=\int_{x V_{\theta}^{\rho} U_{\theta}^{\tau}} \psi\left(p_{\theta}(y)\right) d \lambda_{E, x, s}(y) .
$$

That is, $\sigma_{x, \theta}^{\tau}$ and $\sigma_{x, \theta, s}^{\tau}$ are respectively the push-forwards of $\left.\mu_{H(x)}^{\mathrm{PS}}\right|_{x V_{\theta}^{\rho} U_{\theta}^{\tau}}$ and $\left.\lambda_{E, x, s}\right|_{x V_{\theta}^{\rho} U_{\theta}^{\tau}}$ via the map $p_{\theta}$.

5.2. Energy and Sobolev norms of the projections. Consider the Schwartz space $\mathcal{S}:=\left\{f \in L^{2}\left(x V_{\theta}\right): t^{\alpha} f^{(\beta)} \in L^{2}\left(x V_{\theta}\right)\right\}$, where $\alpha, \beta \in \mathbb{N} \cup\{0\}$ and $f^{(\beta)}$ is the $\beta$-th derivative of $f$. Denote by $\mathcal{S}^{\prime}$ the dual space of $\mathcal{S}$ with the strong dual topology, which is the space of tempered distributions. For $r>0$, we consider the following Sobolev space

$$
H^{r}\left(x V_{\theta}\right):=\left\{f \in \mathcal{S}^{\prime}:(1+|t|)^{r} \hat{f} \in L^{2}\left(x V_{\theta}\right)\right\}
$$

with the norm

$$
\|f\|_{2, r}:=\left\|(1+|t|)^{r} \hat{f}\right\|_{L^{2}\left(x V_{\theta}\right)}
$$

where $\hat{f}$ denotes the Fourier transform of $f$. 
We recall the notion of $\alpha$-energy:

Definition 5.2 ( $\alpha$-energy). For $\alpha>0$ and a Radon measure $\mu$ on $\mathbb{R}^{2}$, the $\alpha$-energy of $\mu$ is given by

$$
I_{\alpha}(\mu):=\int_{\mathbb{R}^{2}} \int_{\mathbb{R}^{2}} \frac{1}{|x-y|^{\alpha}} d \mu(x) d \mu(y) .
$$

It is a standard fact that $I_{\alpha}(\mu)$ can be written as

$$
I_{\alpha}(\mu)=\alpha \int_{\mathbb{R}^{2}} \int_{0}^{\infty} \frac{\mu(B(x, \ell))}{\ell^{1+\alpha}} d \ell d \mu(x)
$$

where $B(x, \ell)$ is the Euclidean disc around $x$ of radius $\ell$.

The $\alpha$-energy of a measure $\mu$ is a useful tool in studying the projections of $\mu$ in various directions. See [28, Proposition 2.2] or [21, Theorem 4.5] for the following theorem:

Theorem 5.3. Let $\nu$ be a Borel probability measure on $\mathbb{R}^{2}$ with compact support. If the 1-energy of $\nu$ is finite, i.e., $I_{1}(\nu)<\infty$, then the following hold:

(1) $p_{\theta *} \nu$ is absolutely continuous with respect to the Lebesgue measure for almost all $\theta$;

(2) there exists $c>1$ (independent of $\nu$ ) such that for any $0<r<\frac{1}{2}$,

$$
c^{-1} I_{1+2 r}(\nu) \leq \int\left\|D\left(p_{\theta *} \nu\right)\right\|_{2, r}^{2} d \theta \leq c I_{1+2 r}(\nu),
$$

where $D\left(p_{\theta *} \nu\right)$ is the Radon-Nikodym derivative of $p_{\theta *} \nu$ with respect to the Lebesgue measure.

Lemma 5.4. Let $Q \subset \mathbb{R}^{2}$ be a compact subset, $c>0$ and $\beta>0$ be fixed. Let $\mathcal{M}$ be a collection of Borel measures on $Q$ such that

$$
\mu(B(x, \ell))<c \cdot \ell^{\beta} \quad \text { for all } \mu \in \mathcal{M}, x \in \operatorname{supp}(\mu) \text { and } \ell>0 .
$$

Then for any $0<\alpha<\beta$,

$$
\sup _{\mu \in \mathcal{M}} I_{\alpha}(\mu)<\infty .
$$

Proof. Fix $0<\alpha<\beta$. We use (5.1). Note that since $\mu(B(x, \ell)) \leq \mu(Q)$, (5.2) has meaning only when $\ell$ is not too big. We use (5.2) only for $0<\ell<1$ and use the upper bound of $\mu(Q)$ for $\ell \geq 1$. We have

$$
\begin{aligned}
\frac{1}{\alpha} I_{\alpha}(\mu) & =\int_{Q} \int_{0}^{1} \frac{\mu(B(x, \ell))}{\ell^{1+\alpha}} d \ell d \mu(x)+\int_{Q} \int_{1}^{\infty} \frac{\mu(B(x, \ell))}{\ell^{1+\alpha}} d \ell d \mu(x) \\
& \leq\left. c \cdot \ell^{\beta-\alpha}\right|_{\ell=0} ^{\ell=1} \cdot \mu(Q)+\left.\ell^{-\alpha}\right|_{\ell=1} ^{\ell=\infty} \cdot \mu(Q)^{2} \\
& =\mu(Q)(c+\mu(Q)) .
\end{aligned}
$$

Now, since $Q$ is compact, the assumption implies that $\sup _{\mu \in \mathcal{M}} \mu(Q)<\infty$. Hence, $I_{\alpha}(\mu)$ is uniformly bounded for all $\mu \in \mathcal{M}$.

Corollary 5.5. Fix $0<\tau \leq \rho$. The following holds for almost all $\theta$ :

(1) $\sigma_{x, \theta}^{\tau}$ is absolutely continuous with respect to the Lebesgue measure on $x V_{\theta}^{\tau}$;

(2) its support has a positive Lebesgue measure;

(3) its Radon-Nikodym derivative satisfies $D\left(\sigma_{x, \theta}^{\tau}\right) \in H^{r}\left(x V_{\theta}^{\tau}\right)$ for any $0<r<$ $\frac{\delta-1}{2}$. 
Proof. It follows from Theorem 4.1 and Lemma 5.4 that for any $0<\alpha<\delta$, $I_{\alpha}\left(\left.\mu_{H\left(x_{0}\right)}^{\mathrm{PS}}\right|_{x_{0} N_{\rho}}\right)<\infty$.

Now, the fact that the support of the projection has positive measure follows from [19, Theorem I]. The other two claims follow from Theorem 5.3 .

$$
\text { We fix } 0<r<\frac{\delta-1}{2} \text { for the rest of this section. }
$$

Terminology 5.6 (PL-direction). If $\theta$ satisfies Corollary 5.5 with respect to $r$, we will call $\theta$ as a "PL" direction for $(x, \tau)$, or simply for $\tau$ when $x$ is fixed.

5.3. Uniform bound for the energy of $\lambda_{E, x, s}, s \geq 1$. In this subsection, we set

$$
\lambda_{E, x, s}^{\dagger}:=\left.\lambda_{E, x, s}\right|_{x N_{\rho}} .
$$

We will show that the collection $\mathcal{M}=\left\{\lambda_{E, x, s}^{\dagger}: s \geq 1\right\}$ of measures on $x N_{\rho}$ satisfies the hypothesis of Lemma 5.4 with $\beta=\delta$. We may consider $\lambda_{E, x, s}^{\dagger}$ as a measure on $\mathbb{R}^{2}$ supported on the $\rho$-ball around the origin.

Since $E$ is a BMS box, $E$ is of the form $x_{0} N_{r_{0}}^{-} A_{r_{0}} N_{r_{0}} M$ for some $0<r_{0}<\rho_{x_{0}}$ where $x_{0}^{ \pm} \in \Lambda(\Gamma)$.

Lemma 5.7. For all $s \geq 1$, we have

$$
x N_{\rho} \cap E a_{-s} \subset\left\{x n \in x N_{\rho}: d\left(x n, P_{H(x)}^{-1}\left(\Lambda(\Gamma)-\left\{x^{-}\right\}\right)\right) \leq e^{-s} r_{0}\right\}
$$

where $P_{H(x)}: \partial_{\infty}\left(\mathbb{H}^{3}\right)-\left\{x^{-}\right\} \rightarrow H(x)$ is defined in the subsection 2.3 and d denotes the Euclidean distance: $d\left(x n_{z}, x n_{z^{\prime}}\right)=\left|z-z^{\prime}\right|$.

Proof. Suppose $x n \in E a_{-s}$, so that $x n=x_{0} \check{n}_{w} a_{t} n_{z} m_{\theta} a_{-s}$ with $|z|<r_{0}$. We may write it as

$$
x n=x_{0} \check{n}_{w} a_{t-s} m_{\theta} n_{e^{-s} e^{2 \pi i \theta} z} .
$$

If we set $y:=x_{0} \check{n}_{w} a_{t-s} m_{\theta}$, then $y^{+}=x_{0}^{+}$. Hence $y^{+} \in \Lambda(\Gamma)$. Since $x n=$ $y n_{e^{-s} e^{2 \pi i \theta} z}$ and $\left|e^{-s} e^{2 \pi i \theta} z\right|<e^{-s} r_{0}$, the claim follows.

Theorem 5.8. There exists $c>0$ such that for all $s \gg 1, y \in \operatorname{supp}\left(\lambda_{E, x, s}^{\dagger}\right)$ and any $\ell>0$,

$$
\lambda_{E, x, s}^{\dagger}(B(y, \ell))<c \cdot \ell^{\delta}
$$

where $B(y, \ell)=\left\{y n_{z}:|z|<\ell\right\}$.

Proof. Since $B(y, 2 \rho)$ contains $x N_{\rho}$, it suffices to show the above for $0<\ell<2 \rho$. Since $\operatorname{supp}\left(\lambda_{E, x, s}\right) \subset E a_{-s} \cap x N_{\rho}$, it follows from Lemma 5.7 that for each $z \in$ $\operatorname{supp}\left(\lambda_{E, x, s}^{\dagger}\right), B\left(z, 3 \rho e^{-s}\right)$ contains $B\left(w, \rho e^{-s}\right)$ for some $w \in H(z)$ with $w^{+} \in \Lambda(\Gamma)$.

Hence by Theorem 4.1 we have

$$
\mu_{H(z)}^{\mathrm{PS}}\left(B\left(z, 3 \rho e^{-s}\right)\right) \geq \mu_{H(z)}^{\mathrm{PS}}\left(B\left(w, \rho e^{-s}\right)\right) \geq c_{0}^{-1}(3 \rho)^{\delta} e^{-\delta s}
$$

where $c_{0}$ is as in Theorem 4.1 with $F_{0}$ being the $\rho$-neighborhood of $\Omega$. Consider the covering of $\operatorname{supp}\left(\lambda_{E, x, s}^{\dagger}\right)$ given by the balls $B\left(z, 3 \rho e^{-s}\right), z \in \operatorname{supp}\left(\lambda_{E, x, s}^{\dagger}\right)$. By the Besicovitch covering lemma we can choose a finite set $J_{s} \subset \operatorname{supp}\left(\lambda_{E, x, s}^{\dagger}\right)$ such that the corresponding finite collection $\left\{B\left(z, 3 \rho e^{-s}\right): z \in J_{s}\right\}$ has multiplicity at most $\kappa$ (independent of $s$ ) and covers $\operatorname{supp}\left(\lambda_{E, x, s}^{\dagger}\right)$. 
Now we consider two cases for $\ell$.

Case 1. $0<\ell \leq e^{-s}$.

In this case, for any $y \in \operatorname{supp}\left(\lambda_{E, x, s}^{\dagger}\right)$, we have

$$
\lambda_{E, x, s}^{\dagger}(B(y, \ell)) \leq \pi e^{(2-\delta) s} \ell^{2} \leq \pi \ell^{\delta} .
$$

Case 2. $e^{-s}<\ell<2 \rho$. Let $J_{y, s}=\left\{z \in J_{s}: B\left(z, 3 \rho e^{-s}\right) \subset B(y, 3 \ell)\right\}$. We have

$$
\begin{aligned}
\lambda_{E, x, s}^{\dagger}(B(y, \ell)) & \leq \sum_{z \in J_{s}}\left\{\lambda_{E, x, s}\left(B\left(z, 3 \rho e^{-s}\right)\right): B\left(z, 3 \rho e^{-s}\right) \cap B(y, \ell) \neq \emptyset\right\} \\
& \leq \sum_{z \in J_{y, s}} \lambda_{E, x, s}\left(B\left(z, 3 \rho e^{-s}\right)\right) \\
& \leq \sum_{z \in J_{y, s}} e^{(2-\delta) s}(3 \rho)^{2} e^{-2 s} \\
& \leq c_{0}(3 \rho)^{2-\delta} \sum_{z \in J_{y, s}} \mu_{H(y)}^{\mathrm{PS}}\left(B\left(z, 3 \rho e^{-s}\right)\right) \\
& \leq \kappa c_{0}(3 \rho)^{2-\delta} \mu_{H(y)}^{\mathrm{PS}}(B(y, 3 \ell)) \\
& \leq 3^{\delta} \kappa c_{0}^{2}(3 \rho)^{2-\delta} \ell^{\delta}
\end{aligned}
$$

Hence for all $0<\ell<2 \rho$ and $y \in \operatorname{supp}\left(\lambda_{E, x, s}\right)$,

$$
\lambda_{E, x, s}^{\dagger}(B(y, \ell)) \leq c_{1} \ell^{\delta}
$$

for some constant $c_{1}>0$ independent of $s \gg 1$.

Therefore by Lemma 5.4, we deduce:

Corollary 5.9. For any $0<\alpha<\delta$,

$$
\sup _{s \gg 1} I_{\alpha}\left(\lambda_{E, x, s}^{\dagger}\right)<\infty
$$

\section{4. $L^{2}$-convergence of projected measures.}

Recall the notation $\sigma_{x, \theta, s_{i}}^{\tau}$ and $\sigma_{x, \theta}^{\tau}$ from Definition 5.1.

Theorem 3.3 is used crucially in the following proposition:

Proposition 5.10. Fix $0<\tau \leq \rho$, a PL-direction $\theta \in M$ for $(x, \tau)$ and a sequence $s_{i} \rightarrow+\infty$. If $\sup _{i}\left\|D\left(\sigma_{x, \theta, s_{i}}^{\tau}\right)\right\|_{2, r}<\infty$, then

$$
D\left(\sigma_{x, \theta, s_{i}}^{\tau} \stackrel{L^{2}\left(x V_{\theta}\right)}{\longrightarrow} D\left(\sigma_{x, \theta}^{\tau}\right) \quad \text { as } i \rightarrow \infty .\right.
$$

Proof. By Theorem 3.3 and the assumption of $x^{ \pm} \in \Lambda(\Gamma),\left.\lambda_{E, x, s_{i}}\right|_{x U_{\theta}^{\rho} V_{\theta}^{\tau}}$ weakly converges to $\left.\mu_{H(x)}^{\mathrm{PS}}\right|_{x U_{\theta}^{\rho} V_{\theta}^{\tau}}$ as $s_{i} \rightarrow \infty$. Therefore, $\sigma_{x, \theta, s_{i}}^{\tau}$ weakly converges to $\sigma_{x, \theta}^{\tau}$ as $i \rightarrow \infty$. Hence, it suffices to show that the collection

$$
\left\{D\left(\sigma_{x, \theta, s_{i}}^{\tau}\right) \in L^{2}\left(x V_{\theta}^{\tau}\right)\right\}
$$

is relatively compact in $L^{2}\left(x V_{\theta}^{\tau}\right)$. Since this collection is uniformly bounded in the Sobolev space $H^{r}\left(x V_{\theta}^{\tau}\right)$ by the assumption, the claim follows from the fact that $H^{r}\left(x V_{\theta}^{\tau}\right)$ embeds compactly in $L^{2}\left(x V_{\theta}^{\tau}\right)$ for any $r>0$ (see [16, Theorem 16.1]).

Recall that by a measure on $[0,2 \pi)$, we mean the Lebesgue measure normalized to be the probability measure. 
Theorem 5.11. Let $s_{i} \rightarrow+\infty$ be a fixed sequence. For any $\varepsilon>0$ and any finite subset $\left\{\tau_{1}, \ldots, \tau_{n}\right\}$ of $(0, \rho]$, there exists a Borel subset $\Theta_{\varepsilon}(x) \subset[0,2 \pi)$, of measure at least $1-\varepsilon$, such that

(1) every $\theta \in \Theta_{\varepsilon}(x)$ is a PL direction for $\left(x, \tau_{\ell}\right)$ for each $1 \leq \ell \leq n$;

(2) for each $\theta \in \Theta_{\varepsilon}(x)$, there exists an infinite subsequence $\left\{s_{j_{i}}\right\}$ (depending on $(x, \theta))$ such that for each $1 \leq \ell \leq n$,

$$
D\left(\sigma_{x, \theta, s_{j_{i}}}^{\tau_{\ell}} \stackrel{L^{2}\left(x V_{\theta}\right)}{\longrightarrow} D\left(\sigma_{x, \theta}^{\tau_{\ell}}\right) .\right.
$$

Proof. Recall that we fixed some $0<r<(\delta-1) / 2$. By Corollary 5.9 and Theorem 5.3, there is a constant $L>1$ such that

$$
\sup _{i} \int\left\|D\left(\sigma_{x, \theta, s_{i}}^{\tau_{\ell}}\right)\right\|_{2, r}^{2} d \theta \leq L \quad \text { for } 1 \leq \ell \leq n .
$$

Hence using Corollary 5.5 and Chebyshev's inequality, we deduce that for any $\varepsilon>0$, there exists some $L_{0}>0$ such that if we let

$$
\Theta_{s_{i}}^{\tau_{\ell}}=\left\{\theta: \theta \text { is a PL direction for }\left(x, \tau_{\ell}\right) \text { and }\left\|D\left(\sigma_{x, \theta, s_{i}}^{\tau_{\ell}}\right)\right\|_{2, r}^{2}<L_{0}\right\},
$$

then for all $i>0$, we have $m\left(\Theta_{s_{i}}^{\tau_{\ell}}\right)>1-\frac{\varepsilon}{2 n}$. Let $\Theta_{i}=\cap_{\ell} \Theta_{s_{i}}^{\tau_{\ell}}$ and let $\Theta=\lim \sup _{i} \Theta_{i}$. Then $m(\Theta)>1-\varepsilon$. For $\theta \in \Theta, \theta$ lies in infinitely many of $\Theta_{i}$ 's, i.e., $\theta \in \Theta_{j_{i}}$ for some infinite subsequence $\left\{j_{i}\right\}$. Hence, the claim follows from Proposition 5.10 applied to $\left\{s_{j_{i}}\right\}$.

5.5. Key lemma on the projections of $\lambda_{E, x, s}^{\dagger}$. The following is the key technical lemma in the proof of the window theorem.

Lemma 5.12 (Key Lemma). Fix $0<\tau<\rho$ and a sequence $s_{i} \rightarrow+\infty$. For any $\varepsilon>0$, there exists a Borel subset $\Theta_{\varepsilon}(x) \subset[0,2 \pi)$ of measure at least $1-\varepsilon$ such that if $\theta \in \Theta_{\varepsilon}(x)$ and $E_{i} m_{\theta}^{-1} \subset X$ is a sequence of Borel subsets satisfying

$$
\lambda_{E, x, s_{i}}\left(x N_{\tau}-E_{i} m_{\theta}^{-1}\right) \rightarrow 0,
$$

then there is an infinite subsequence $\left\{s_{j_{i}}\right\}$ such that for any Borel subset $O_{\theta}(x) \subset$ $x V_{\theta}^{\rho}$,

$$
\limsup _{i} \sigma_{x, \theta, s_{j_{i}}}^{\rho}\left(O_{\theta}(x)-p_{\theta}\left(E_{i} m_{\theta}^{-1} \cap x N_{\tau}\right)\right) \leq \sigma_{x, \theta}^{\rho}\left\{t \in O_{\theta}(x): D\left(\sigma_{x, \theta}^{\tau}\right)(t)=0\right\} .
$$

By Theorem 5.11, the Key Lemma follows from the following lemma. Observe that this is a rather strong control on the conditional measures, as one can easily construct counter-examples in a general setting. Here our $L^{2}$ - convergence result of the projection measures to a "rich" measure is crucially used.

Lemma 5.13. Fix $0<\tau<\rho$ and a PL direction $\theta \in[0,2 \pi)$, simultaneously for $(x, \tau)$ and $(x, \rho)$. Let $W_{i} m_{\theta}^{-1} \subset x N_{\tau}$ be a sequence of Borel subsets and $\left\{s_{i}\right\}$ be a sequence tending to infinity. Assume the following holds as $i \rightarrow \infty$ :

(1) $D\left(\sigma_{x, \theta, s_{i}}^{\tau}\right) \stackrel{L^{2}\left(x V_{\theta}\right)}{\longrightarrow} D\left(\sigma_{x, \theta}^{\tau}\right)$;

(2) $D\left(\sigma_{x, \theta, s_{i}}^{\rho}\right) \stackrel{L^{2}\left(x V_{\theta}\right)}{\longrightarrow} D\left(\sigma_{x, \theta}^{\rho}\right)$;

(3) $\lambda_{E, x, s_{i}}\left(x N_{\tau}-W_{i} m_{\theta}^{-1}\right) \rightarrow 0$.

Then for any Borel subset $O_{\theta}(x) \subset x V_{\theta}^{\rho}$,

$$
\limsup _{i} \sigma_{x, \theta, s_{i}}^{\rho}\left(O_{\theta}(x)-p_{\theta}\left(W_{i} m_{\theta}^{-1}\right)\right) \leq \sigma_{x, \theta}^{\rho}\left\{t \in O_{\theta}(x): D\left(\sigma_{x, \theta}^{\tau}\right)(t)=0\right\} .
$$


Proof. Set $\mathcal{P}^{\tau}:=\left\{t \in O_{\theta}(x): D\left(\sigma_{x, \theta}^{\tau}\right)(t)>0\right\}$ and

$$
\mathcal{L}_{i}^{\tau}:=p_{\theta}\left(O_{\theta}(x) U_{\theta}^{\tau} \cap W_{i} m_{\theta}^{-1}\right)=O_{\theta}(x) \cap p_{\theta}\left(W_{i} m_{\theta}^{-1}\right) .
$$

For $n_{0}>1$, define

$$
\Sigma_{n_{0}}=\left\{t \in \mathcal{P}^{\tau}: D\left(\sigma_{x, \theta}^{\tau}\right)(t) \geq \frac{1}{n_{0}}, D\left(\sigma_{x, \theta}^{\rho}\right)(t)<n_{0}\right\} .
$$

Let $\varepsilon>0$ be arbitrary. There exists $n_{0}=n_{0}(\varepsilon)>1$ such that

$$
\sigma_{x, \theta}^{\rho}\left(\Sigma_{n_{0}}\right)>(1-\varepsilon) \sigma_{x, \theta}^{\rho}\left(\mathcal{P}^{\tau}\right) .
$$

Since $D\left(\sigma_{x, \theta, s_{i}}^{\tau}\right) \rightarrow D\left(\sigma_{x, \theta}^{\tau}\right)$ in $L^{2}\left(x V_{\theta}\right)$, denoting by $\lambda$ the Lebesgue measure on $x V_{\theta}$, we have

$$
\begin{aligned}
\left|\sigma_{x, \theta}^{\tau}\left(O_{\theta}(x)-\mathcal{L}_{i}^{\tau}\right)-\sigma_{x, \theta, s_{i}}^{\tau}\left(O_{\theta}(x)-\mathcal{L}_{i}^{\tau}\right)\right| & \leq \int_{x V_{\theta}}\left|D\left(\sigma_{x, \theta}^{\tau}\right)-D\left(\sigma_{x, \theta, s_{i}}^{\tau}\right)\right| d \lambda \\
& \leq\left\|D\left(\sigma_{x, \theta}^{\tau}\right)-D\left(\sigma_{x, \theta, s_{i}}^{\tau}\right)\right\|_{2} \cdot \lambda\left(x V_{\theta}\right)^{1 / 2} \rightarrow 0 .
\end{aligned}
$$

Since $\sigma_{x, \theta, s_{i}}^{\tau}\left(O_{\theta}(x)-\mathcal{L}_{i}^{\tau}\right) \leq \lambda_{E, x, s_{i}}\left(x N_{\rho}-W_{i} m_{\theta}^{-1}\right) \rightarrow 0$ by the assumption on $W_{i} m_{\theta}$, it follows now that there is some $i_{0}=i_{0}\left(n_{0}\right)$ such that for all $i \geq i_{0}$,

$$
\sigma_{x, \theta}^{\tau}\left(O_{\theta}(x)-\mathcal{L}_{i}^{\tau}\right)<\frac{\varepsilon}{n_{0}^{2}} .
$$

Note that for any set $\Upsilon \subset \Sigma_{n_{0}}$ with $\sigma_{x, \theta}^{\tau}(\Upsilon)<\frac{\varepsilon}{n_{0}^{2}}$, we have $\sigma_{x, \theta}^{\rho}(\Upsilon)<\varepsilon$. To see this, note that if

$$
\frac{1}{n_{0}} \lambda(\Upsilon) \leq \int_{\Upsilon} D\left(\sigma_{x, \theta}^{\tau}, t\right) d \lambda(t) \leq \frac{\varepsilon}{n_{0}^{2}}
$$

then $\lambda(\Upsilon) \leq \frac{\varepsilon}{n_{0}}$ and hence

$$
\sigma_{x, \theta}^{\rho}(\Upsilon)=\int_{\Upsilon} D\left(\sigma_{x, \theta}^{\rho}\right)(t) d \lambda(t) \leq n_{0} \lambda(\Upsilon) \leq \frac{\varepsilon}{n_{0}} \leq \varepsilon
$$

Therefore, we have

$$
\begin{aligned}
\sigma_{x, \theta}^{\rho}\left(O_{\theta}(x)-\mathcal{L}_{i}^{\tau}\right) & \leq \sigma_{x, \theta}^{\rho}\left(\left(O_{\theta}(x)-\mathcal{L}_{i}^{\tau}\right) \cap \Sigma_{n_{0}}\right)+\sigma_{x, \theta}^{\rho}\left(\left(O_{\theta}(x)-\mathcal{L}_{i}^{\tau}\right) \cap\left(O_{\theta}(x)-\Sigma_{n_{0}}\right)\right) \\
& \leq \varepsilon+\varepsilon \cdot \sigma_{x, \theta}^{\rho}\left(\mathcal{P}_{\theta}^{\tau}\right)+\sigma_{x, \theta}^{\rho}\left(O_{\theta}(x)-\mathcal{P}_{\theta}^{\tau}\right) .
\end{aligned}
$$

Since $\varepsilon>0$ is arbitrary,

$$
\limsup _{i} \sigma_{x, \theta}^{\rho}\left(O_{\theta}(x)-\mathcal{L}_{i}^{\tau}\right) \leq \sigma_{x, \theta}^{\rho}\left(O_{\theta}(x)-\mathcal{P}_{\theta}^{\tau}\right) .
$$

Now since $D\left(\sigma_{x, \theta, s_{i}}^{\rho}\right) \rightarrow D\left(\sigma_{x, \theta}^{\rho}\right)$ in $L^{2}\left(x V_{\theta}^{\rho}\right)$, we have

$$
\left|\sigma_{x, \theta}^{\rho}\left(O_{\theta}(x)-\mathcal{L}_{i}^{\tau}\right)-\sigma_{x, \theta, s_{i}}^{\rho}\left(O_{\theta}(x)-\mathcal{L}_{i}^{\tau}\right)\right| \leq \int_{x V_{\theta}}\left|D\left(\sigma_{x, \theta, s_{i}}^{\rho}\right)-D\left(\sigma_{x, \theta}^{\rho}\right)\right| d \lambda \rightarrow 0 .
$$

Combined with (5.3), this implies that

$$
\limsup _{i} \sigma_{x, \theta, s_{i}}^{\rho}\left(O_{\theta}(x)-\mathcal{L}_{i}^{\tau}\right) \leq \sigma_{x, \theta}^{\rho}\left(O_{\theta}(x)-\mathcal{P}_{\theta}^{\tau}\right) .
$$




\section{ReCURREnce Properties of BMS ANd BR MeAsures}

6.1. Theorems of Marstrand on Hausdorff measures. Let $\Lambda \subset \mathbb{R}^{2}$. The $s$-dimensional Hausdorff measure of $\Lambda$ is defined to be

$$
\mathcal{H}^{s}(\Lambda)=\inf _{\eta \downarrow 0} \mathcal{H}_{\eta}^{s}(\Lambda)
$$

where $\mathcal{H}_{\eta}^{s}(\Lambda):=\left\{\sum_{i} d\left(W_{i}\right)^{s}: \Lambda \subset \cup_{i=1}^{\infty} W_{i}, \quad d\left(W_{i}\right) \leq \eta\right\}$ and $d\left(W_{i}\right)$ denotes the diameter of $W_{i}$.

The Hausdorff dimension of $\Lambda$ is

$$
\operatorname{dim}(\Lambda)=\sup \left\{s: \mathcal{H}^{s}(\Lambda)>0\right\}=\inf \left\{s: \mathcal{H}^{s}(\Lambda)=\infty\right\} .
$$

A set $\Lambda$ is called an $s$-set if $0<\mathcal{H}^{s}(\Lambda)<\infty$. Following Marstrand 19, a point $\xi \in \Lambda$ is called a condensation point for $\Lambda$ if $\xi$ is a limit point from $(\xi, \theta) \cap \Lambda$ for almost all $\theta$ where $(\xi, \theta)$ denotes the ray through $\xi$ lying in the direction $\theta$.

Let $\Lambda$ be an $s$-set in the following three theorems:

Theorem 6.1. 19, Theorem 7.2] If $s>1, \mathcal{H}^{s}$-almost all points in $\Lambda$ are condensation points for $\Lambda$.

Theorem 6.2. [19, Lemma 19] If $s>1$, almost every lines $L$ through $\mathcal{H}^{s}$-almost all points in $\Lambda$ intersect $\Lambda$ in a set of dimension $s-1$.

Theorem 6.3. 19, Theorem II] If $s \leq 1$, then the projections of $\Lambda$ have Hausdorff dimension $s$ for almost all directions.

6.2. $U$-conservativity of $m^{\mathrm{BR}}$. In the rest of this section, we assume that $\Gamma$ is convex cocompact.

Theorem 6.4. 35] For $x \in G$, the measure $\mu_{H^{+}(x)}^{\mathrm{PS}}$ on $x N$ is a $\delta$-dimensional Hausdorff measure supported on the set $\left\{x n \in H^{+}(x):(x n)^{+} \in \Lambda(\Gamma)\right\}$. Furthermore, this is a positive and locally finite measure on $x N$.

For $U=\left\{u_{t}=\left(\begin{array}{cc}1 & 0 \\ t & 1\end{array}\right): t \in \mathbb{R}\right\}$, we recall the definition of a conservative action:

Definition 6.5 (Conservative action). Let $\mu$ be a locally finite $U$-invariant measure on $X$. The $U$-action on $X$ is conservative for $\mu$ if one of the following equivalent conditions holds:

(1) for every positive Borel function $\psi$ of $X$,

$$
\int_{t \in \mathbb{R}} \psi\left(x u_{t}\right) d t=\infty \text { for a.e. } x \in X
$$

(2) for any Borel subset $B$ of $X$ with $\mu(B)>0$,

$$
\int_{t \in \mathbb{R}} \chi_{B}\left(x u_{t}\right) d t=\infty \quad \text { for a.e. } x \in B
$$

The following is Maharam's recurrence theorem (cf. [1, 1.1.7]).

Lemma 6.6. If there is a measurable subset $B \subset X$ with $0<m^{\mathrm{BR}}(B)<\infty$ such that for almost all $x \in X, \int_{0}^{\infty} \chi_{B}\left(x u_{t}\right) d t=\infty$, then $U$ is conservative for $m^{\mathrm{BR}}$.

Theorem 6.7. If $\delta>1$, then $U$ is conservative for $m^{\mathrm{BR}}$. 
Proof. Recall the notation $\Omega=\operatorname{supp}\left(m^{\mathrm{BMS}}\right)$ and $\Omega_{\mathrm{BR}}=\operatorname{supp}\left(m^{\mathrm{BR}}\right)$. Set

$$
\mathcal{F}:=\left\{x \in X: x^{-} \in \Lambda(\Gamma), x u_{t} \notin \Omega \text { for all large } t \gg_{x} 1\right\} .
$$

Hence $x \in \mathcal{F}$ means $\left(x u_{t}\right)^{+} \notin \Lambda(\Gamma)$ for all large $t \gg_{x} 1$. We claim that

$$
m^{\mathrm{BR}}(\mathcal{F})=0 .
$$

Suppose not. Then by the Fubini theorem, there is a set $\mathcal{O} \subset \Omega_{\mathrm{BR}}$ with $m^{\mathrm{BR}}(\mathcal{O})>0$ such that for all $x \in \mathcal{O}, x m_{\theta} \in \mathcal{F}$ for a positive measurable subset of $\theta$ 's. Note that $\nu_{o}\left(\left\{x^{-} \in \Lambda(\Gamma): x \in \mathcal{O}\right\}\right)>0$ where $\nu_{o}$ is the PS measure on $\Lambda(\Gamma)$. Fix $\xi_{0} \notin \Lambda(\Gamma)$ and identify $\partial\left(\mathbb{H}^{3}\right)-\left\{\xi_{0}\right\}$ with $\mathbb{R}^{2}$. Since $\left.\nu_{o}\right|_{\partial\left(\mathbb{H}^{3}\right)-\left\{\xi_{0}\right\}}$ is equivalent to the $\delta$-dimensional Hausdorff measure $\mathcal{H}^{\delta}$ on $\Lambda(\Gamma) \subset \mathbb{R}^{2}$ by Theorem 6.4, we have $\mathcal{H}^{\delta}\left\{x^{-} \in \Lambda(\Gamma): x \in \mathcal{O}\right\}>0$. Note that $L_{\theta}(x):=\left\{\left(x m_{\theta} u_{t}\right)^{+} \in \mathbb{R}^{2}=\partial\left(\mathbb{H}^{3}\right)-\{\xi\}:\right.$ $t \geq 0\}$ is the line segment connecting $x^{+}($at $t=0)$ and $x^{-}($at $t=\infty)$. Hence $x \in \mathcal{O}$ implies that $x^{-}$is not a limit point of the intersection $L_{\theta}(x) \cap \Lambda(\Gamma)$ for a positive set of directions $\theta$. This contradicts Theorem 6.1 and proves the claim 66.1).

Let $\mathcal{O}$ be an $r$-neighborhood of $\Omega$ for some small $r>0$. If $x \in X-\mathcal{F}$, then $x u_{t} \in \Omega$ and $x u_{t+s} \in \mathcal{O}$ for all $|s|<r$. Hence, if $x u_{t_{i}} \in \Omega$ for an unbounded sequence $t_{i}, \int_{t \in \mathbb{R}} \chi_{\mathcal{O}}\left(x u_{t}\right) d t=\infty$. As $m^{\mathrm{BR}}(\mathcal{F})=0$ and $0<m^{\mathrm{BR}}(\mathcal{O})<\infty$, this implies the claim by Lemma 6.6

6.3. Leafwise measures. Let $W$ be a closed connected subgroup of $N$. Let $\mathcal{M}_{\infty}(W)$ denote the space of locally finite measures on $W$ with the smallest topology so that the map $\nu \mapsto \int \psi d \nu$ is continuous for all $\psi \in C_{c}(W)$ (the weak* topology). A locally finite Borel measure $\mu$ on $X$ gives rise to a system of locally finite measures $\left[\mu_{x}^{W}\right] \in \mathcal{M}_{\infty}(W)$, unique up to normalization, called the leafwise measures or conditional measures on $W$-orbits. There is no canonical way of normalizing these measures. For our purpose here, we fix a normalization so that $\mu_{x}^{W}\left(N_{1} \cap W\right)=1$. With this normalization, the assignment $x \mapsto \mu_{x}^{W}$, is a Borel map, furthermore, for a full measure subset $X^{\prime}$ of $X, \mu_{x u}^{W}=u . \mu_{x}^{W}$ for every $x, x u \in X^{\prime}$; for a comprehensive account on leafwise measures we refer the reader to 7 .

In the case when $W=N$, we have $\mu_{H(x)}^{\mathrm{PS}}=\mu_{x}^{\mathrm{PS}}$ and $\mu_{H(x)}^{\mathrm{Leb}}=\lambda_{x}$, which are precisely the $N$-leafwise measures of the BMS and BR measures respectively, up to normalization. We will be considering the $U$ leafwise measures of $m^{\mathrm{BMS}}$ as well as of $\mu_{E, s}^{\mathrm{BR}}$.

We will use the following simple lemma.

Lemma 6.8. Let $\mu$ be a locally finite $M$-invariant measure on $X$. For any $0<$ $\tau \ll 1$, and any $0 \leq \theta<\pi$, we have

$$
\left|\mu_{x m_{\theta}}^{U^{\tau}}\right|=\left|\mu_{x}^{U_{\theta}^{\tau}}\right|
$$

for $\mu$ a.e. $x \in X$.

Proof. Since $\mu$ is $M$-invariant, for $T_{\rho}=A_{\rho} \check{N}_{\rho} M_{\rho}$, we have

$$
\frac{\left|\mu_{x}^{U_{\theta}^{\tau}}\right|}{\left|\mu_{x m_{\theta}}^{U^{\tau}}\right|}=\lim _{\rho \rightarrow 0} \frac{\mu\left(x m_{\theta} U^{\tau} m_{\theta}^{-1}\left(m_{\theta} V_{\rho} T_{\rho} m_{\theta}^{-1}\right)\right)}{\mu\left(x m_{\theta} U^{\tau}\left(V_{\rho} T_{\rho}\right)\right)}=1 .
$$


6.4. Recurrence for $m^{\text {BMS }}$. Since the frame flow is mixing by Theorem 2.9 with respect to $m^{\mathrm{BMS}}$, we have:

Proposition 6.9. For any non-trivial $a \in A, m^{\mathrm{BMS}}$ is a-ergodic.

Theorem 6.10. Let $\delta>1$. For a.e. $x \in \Omega,\left(m^{\mathrm{BMS}}\right)_{x}^{U}$ is atom-free.

Proof. Setting $\mathcal{F}:=\left\{x \in \Omega:\left(m^{\mathrm{BMS}}\right)_{x}^{U}\right.$ has an atom $\}$, we first claim that $m^{\mathrm{BMS}}(\mathcal{F})=0$. Suppose not. Fix any non-trivial $a \in A$. Since $U$ is normalized by $a, \mathcal{F}$ is $a$-invariant. Hence $m^{\mathrm{BMS}}(\mathcal{F})=1$ by Proposition 6.9. Using the Poincare recurrence theorem, it can be shown that

$$
\mathcal{F}^{\prime}:=\left\{x \in \Omega:\left(m^{\mathrm{BMS}}\right)_{x}^{U} \text { is the dirac measure at } e\right\}
$$

has a full measure in $\Omega$ (cf. [13], [15, Theorem 7.6]).

Since for any $x \in \Omega, \mu_{x}^{\mathrm{PS}}$ is a positive $\delta$-dimensional Hausdorff measure on $\{x n \in$ $\left.H(x):(x n)^{+} \in \Lambda(\Gamma)\right\}$ by Theorem 6.4 and $\left(m^{\mathrm{BMS}}\right)_{x}^{U}=\left(\mu_{H(x)}^{\mathrm{PS}}\right)_{x}^{U}$ for a.e. $x \in \Omega$, it follows that for a.e. $x \in \Omega,\left(\mu_{H(x)}^{\mathrm{PS}}\right)_{x}^{U}$ is the Dirac measure at $e$. By the Fubini theorem, there exist $x \in \Omega$ and a measurable subset $D_{0} \subset H(x)$ with $\mu_{H(x)}^{\mathrm{PS}}\left(D_{0}\right)>0$ such that for each $y \in D_{0},\left(\mu_{H\left(y m_{\theta}\right)}^{\mathrm{PS}}\right)_{y m_{\theta}}^{U}$ is the dirac measure at $y m_{\theta}$ for a positive measurable subset of $m_{\theta}$ 's. For $s \geq 0$, denote by $\mathcal{H}^{s}$ the $s$-dimensional Hausdorff measure on $\Lambda(\Gamma)-\left\{x^{-}\right\}$; so $\mathcal{H}^{\delta}=\mu_{H(x)}^{\mathrm{PS}}$. In the identification of $H(x)$ with $\mathbb{R}^{2}$ via the map $y \mapsto y^{+}$, this implies that there is a subset $D_{0}^{\prime} \subset \Lambda(\Gamma)-\left\{x^{-}\right\} \subset \mathbb{R}^{2}$ with $\mathcal{H}^{\delta}\left(D_{0}^{\prime}\right)>0$ such that for all $\xi \in D_{0}^{\prime}$, there is a positive measurable subset of lines $L$ through $\xi$ such that $0<\mathcal{H}^{0}\left(\left(\Lambda(\Gamma)-\left\{x^{-}\right\}\right) \cap L\right)<\infty$. This contradicts Theorem 6.2 which implies that $\left(\Lambda(\Gamma)-\left\{x^{-}\right\}\right) \cap L$ has dimension $\delta-1>0$ for almost all lines $L$ through $\xi$.

Corollary 6.11. If $\delta>1, m^{\mathrm{BMS}}$ is $U$-recurrent, i.e., for any measurable subset $B$ of $X,\left\{t: x u_{t} \in B\right\}$ is unbounded for a.e. $x \in B$.

Proof. By [7, Theorem 7.6], Theorem 6.10 implies that $\left(m^{\mathrm{BMS}}\right)_{x}^{U}$ is infinite for a.e. $x$. [7, Theorem 6.25] implies the claim.

6.5. Doubling for the $\left(\mu^{\mathrm{BMS}}\right)_{x}^{U}$. As before, we assume $\left|m^{\mathrm{BMS}}\right|=1$. Since $\Omega$ is a compact subset, we have

$$
\rho:=\frac{1}{2} \inf \left\{\rho_{x}: x \in \Omega\right\}>0 .
$$

Fix a small number $\varepsilon>0$. It follows from Theorem 6.10 that there exist $0<$ $\beta=\beta(\varepsilon) \ll \rho$ and a compact subset $\Omega_{\varepsilon}^{\prime} \subset \Omega$ with $m^{\mathrm{BMS}}\left(\Omega_{\varepsilon}^{\prime}\right)>1-\frac{\varepsilon^{2}}{2}$ such that

$$
\left(m^{\mathrm{BMS}}\right)_{x}^{U}[-3 \beta, 3 \beta]<\frac{1}{2}\left(m^{\mathrm{BMS}}\right)_{x}^{U}[-(\rho-\beta), \rho-\beta] \quad \text { for all } x \in \Omega_{\varepsilon}^{\prime} .
$$

Since the covering $\left\{x B_{\tau}: x \in \Omega, \tau>0\right\}$ admits a disjoint subcovering of $\Omega$ with full BMS measure (see [20, Theorem 2.8]), there exist $x_{0}=x_{0}(\varepsilon) \in \Omega_{\varepsilon}^{\prime}$ and $0<\tau<\beta(\varepsilon)$ such that for $B_{x_{0}}(\tau):=x_{0} \check{N}_{\tau} A_{\tau} M N_{\tau}$,

$$
m^{\mathrm{BMS}}\left(B_{x_{0}}(\tau) \cap \Omega_{\varepsilon}^{\prime}\right)>\left(1-\frac{\varepsilon^{2}}{2}\right) \cdot m^{\mathrm{BMS}}\left(B_{x_{0}}(\tau)\right) .
$$

We fix $x_{0} \in \Omega_{\varepsilon}^{\prime}$ and $\tau>0$ for the rest of this section. 
Recall the notation $T_{\tau}=\check{N}_{\tau} A_{\tau} M$, so that $B_{x_{0}}(\tau)=x_{0} T_{\tau} N_{\tau}$. Set $\nu=\nu_{x_{0} T_{\tau}}$ for simplicity. Using Theorem 6.10, we will prove:

Theorem 6.12. Let $\delta>1$. Let $c_{0}>1$ be as in Theorem 4.1 where $F_{0}$ is the $2 \rho$ neighborhood of $\Omega$. Then there exists a Borel subset $\Xi_{\varepsilon}^{\mathrm{PS}}\left(x_{0}\right) \subset x_{0} T_{\tau}$ which satisfies the following properties:

(i) $\nu\left(\Xi_{\varepsilon}^{\mathrm{PS}}\left(x_{0}\right)\right) \geq\left(1-c_{0}^{4} \cdot \varepsilon\right) \nu\left(x_{0} T_{\tau}\right)$;

(ii) for any $x m_{\theta} \in \Xi_{\varepsilon}^{\mathrm{PS}}\left(x_{0}\right)$, with $\theta$ a $\mathrm{PL}$ direction for $(x, \tau)$, there exists a Borel subset $O_{\theta}(x)$ of the set $\left\{t \in x V_{\theta}: D\left(\sigma_{x, \theta}^{\tau}\right)(t)>0\right\}$ such that

$$
\mu_{x}^{\mathrm{PS}}\left(O_{\theta}(x) U_{\theta}^{\rho}\right) \geq 2 \mu_{x}^{\mathrm{PS}}\left(O_{\theta}(x) U_{\theta}^{2 \tau}\right) \geq \frac{\tau^{\delta}}{4 c_{0}} .
$$

Despite the rather complicated formulation of this theorem, which is tailored towards our application later, the theorem is intuitively clear. Indeed, $B_{x_{0}}(\tau)$ is chosen so that for "most" BMS points, we have (6.3). On the other hand, in view of Corollary [5.5, for a PL direction $\theta$, the Radon-Nikodym derivative $D\left(\sigma_{x, \theta}^{\tau}\right)$ is positive $\sigma_{x, \theta}^{\tau}$-almost everywhere. Therefore, by Fubini's theorem, for "most" BMS points $x \in B_{x_{0}}(\tau)$, "most" points in $x N_{\rho}$ satisfy both (6.3) and the non-vanishing of the Radon-Nikodym derivative implies Theorem 6.12. The precise treatment of the above sketch of the proof is given in the rest of this subsection.

Lemma 6.13. Let $x m_{\theta} \in x_{0} T_{\tau}$. For any Borel subset $O_{\theta}^{\prime}(x) \subset p_{\theta}\left(x N_{\tau} \cap \Omega_{\varepsilon}^{\prime} m_{\theta}^{-1}\right)$,

$$
\mu_{x}^{\mathrm{PS}}\left(O_{\theta}^{\prime}(x) U_{\theta}^{\rho}\right) \geq 2 \mu_{x}^{\mathrm{PS}}\left(O_{\theta}^{\prime}(x) U_{\theta}^{2 \tau}\right) .
$$

Proof. If $t \in p_{\theta}\left(x N_{\tau} \cap \Omega_{\varepsilon}^{\prime} m_{\theta}^{-1}\right)$ and hence $t=x v_{\theta}$ for $v_{\theta} \in V_{\theta}$ where $z_{t} m_{\theta}^{-1}=x v_{\theta} u_{\theta}$ for $z_{t} \in \Omega_{\varepsilon}^{\prime}$, we can write it as $t m_{\theta}=z_{t} m_{\theta}^{-1} u_{\theta}^{-1} m_{\theta}=z_{t} u_{0}$ for some $u_{0} \in U^{\tau}$. Hence, by (6.3),

$$
\left|\mu_{t m_{\theta}}^{U^{\rho}}\right| \geq\left|\mu_{z_{t}}^{U^{\rho-\tau}}\right| \geq 2\left|\mu_{z_{t}}^{U^{3 \tau}}\right| \geq 2\left|\mu_{t m_{\theta}}^{U^{2 \tau}}\right| .
$$

Using this and since $O_{\theta}^{\prime}(x) \subset p_{\theta}\left(x N_{\tau} \cap \Omega_{\varepsilon}^{\prime} m_{\theta}^{-1}\right)$, we have

$$
\begin{aligned}
\mu_{x}^{\mathrm{PS}}\left(O_{\theta}^{\prime}(x) U_{\theta}^{\rho}\right) & =\int_{O_{\theta}^{\prime}(x)}\left|\mu_{t}^{U_{\theta}^{\rho}}\right| d \sigma_{x, \theta}^{\rho}(t)=\int_{O_{\theta}^{\prime}(x)}\left|\mu_{t m_{\theta}}^{U^{\rho}}\right| d \sigma_{x, \theta}^{\rho}(t) \\
& \geq 2 \int_{O_{\theta}^{\prime}(x)}\left|\mu_{t m_{\theta}}^{U^{2 \tau}}\right| d \sigma_{x, \theta}^{\rho}(t)=2 \int_{O_{\theta}^{\prime}(x)}\left|\mu_{t}^{U_{\theta}^{2 \tau}}\right| d \sigma_{x, \theta}^{\rho}(t) \\
& =2 \mu_{x}^{\mathrm{PS}}\left(O_{\theta}^{\prime}(x) U_{\theta}^{2 \tau}\right) .
\end{aligned}
$$

Lemma 6.14. There exists a compact subset $\Omega_{\varepsilon} \subset \Omega_{\varepsilon}^{\prime}$ with $m^{\mathrm{BMS}}\left(\Omega_{\varepsilon}\right)>1-\varepsilon^{2}$ such that

$$
m^{\mathrm{BMS}}\left(B_{x_{0}}(\tau) \cap \Omega_{\varepsilon}\right)>\left(1-\varepsilon^{2}\right) \cdot m^{\mathrm{BMS}}\left(B_{x_{0}}(\tau)\right)
$$

and that

$$
p_{\theta}\left(x N_{\tau} \cap \Omega_{\varepsilon} m_{\theta}^{-1}\right) \subset\left\{t \in x V_{\theta}: D\left(\sigma_{x, \theta}^{\tau}\right)(t)>0\right\}
$$

for all $x m_{\theta} \in x_{0} T_{\tau}$ with $\theta$ a PL-direction for $(x, \tau)$. 
Proof. Setting $x V_{\theta}^{\prime}:=\left\{t \in x V_{\theta}: D\left(\sigma_{x, \theta}^{\tau}, t\right)=0\right\}$, we have

$$
\begin{aligned}
m^{\mathrm{BMS}}\left(\cup_{x m_{\theta} \in x_{0} T_{\tau}} x V_{\theta}^{\prime} U_{\theta}^{\tau}\right) & =\int_{x m_{\theta} \in x_{0} T_{\tau}} \mu_{x}^{\mathrm{PS}}\left(x V_{\theta}^{\prime} U_{\theta}^{\tau}\right) d \nu\left(x m_{\theta}\right) \\
& =\int_{x m_{\theta} \in x_{0} T_{\tau}} \int_{t \in x V_{\theta}^{\prime}}\left|\left(\mu_{x}^{\mathrm{PS}}\right)_{x}^{U_{\theta}^{\tau}}\right| d \sigma_{x, \theta}^{\tau}(t) d \nu\left(x m_{\theta}\right)=0 .
\end{aligned}
$$

Hence, there exists an open subset $\mathcal{O}_{\varepsilon}$ of $B_{x_{0}}(\tau)$ which contains the subset $\cup_{x m_{\theta} \in x_{0} T_{\tau}} x V_{\theta}^{\prime} U_{\theta}^{\tau}$ and $m^{\mathrm{BMS}}\left(\mathcal{O}_{\varepsilon}\right) \leq \frac{\varepsilon^{2}}{2} \cdot m^{\mathrm{BMS}}\left(B_{x_{0}}(\tau)\right)$. Now set $\Omega_{\varepsilon}:=\Omega_{\varepsilon}^{\prime}-\mathcal{O}_{\varepsilon}$. It is easy to check that this $\Omega_{\varepsilon}$ satisfies the claim.

We set

$$
\Xi_{\varepsilon}^{\mathrm{PS}}\left(x_{0}\right):=\left\{x \in x_{0} T_{\tau}: \mu_{x}^{\mathrm{PS}}\left(x N_{\tau} \cap \Omega_{\varepsilon}\right)>(1-\varepsilon) \mu_{x}^{\mathrm{PS}}\left(x N_{\tau}\right)\right\} .
$$

Lemma 6.15. We have

$$
\nu\left(\Xi_{\varepsilon}^{\mathrm{PS}}\left(x_{0}\right)\right) \geq\left(1-c_{0}^{4} \cdot \varepsilon\right) \nu\left(x_{0} T_{\tau}\right) .
$$

Proof. Set $b_{1}:=\inf _{x \in x_{0} T_{\tau}} \frac{\mu_{x_{0}}^{\mathrm{PS}}\left(x_{0} N_{\tau}\right)}{\mu_{x}^{\mathrm{PS}}\left(x N_{\tau}\right)}$ and $b_{2}:=\sup _{x \in x_{0} T_{\tau}} \frac{\mu_{x_{0}}^{\mathrm{PS}}\left(x_{0} N_{\tau}\right)}{\mu_{x}^{\mathrm{PS}}\left(x N_{\tau}\right)}$. Since $x_{0}^{+} \in$ $\Lambda(\Gamma)$ and $x_{0}^{+}=x^{+}$, we have $x^{+} \in \Lambda(\Gamma)$ and hence it follows from Theorem 4.1 that $c_{0}^{-4}<\frac{b_{2}}{b_{1}}<c_{0}^{4}$.

Note that

$$
\begin{aligned}
& m^{\mathrm{BMS}}\left(B_{x_{0}}(\tau) \cap \Omega_{\varepsilon}\right) \\
& \quad \leq \int_{\Xi_{\varepsilon}^{\mathrm{PS}}\left(x_{0}\right)} \mu_{y}^{\mathrm{PS}}\left(y N_{\tau}\right) d \nu(y)+(1-\varepsilon) \int_{x_{0} T_{\tau}-\Xi_{\varepsilon}^{\mathrm{PS}}\left(x_{0}\right)} \mu_{y}^{\mathrm{PS}}\left(y N_{\tau}\right) d \nu(y) \\
& \quad \leq m^{\mathrm{BMS}}\left(B_{x_{0}}(\tau)\right)-\frac{\varepsilon}{b_{2}} \nu\left(x_{0} T_{\tau}-\Xi_{\varepsilon}^{\mathrm{PS}}\left(x_{0}\right)\right) \cdot \mu_{x_{0}}^{\mathrm{PS}}\left(x_{0} N_{\tau}\right) .
\end{aligned}
$$

By Lemma 6.14, it follows that

$$
\varepsilon^{2} \cdot m^{\mathrm{BMS}}\left(B_{x_{0}}(\tau)\right) \geq \frac{\varepsilon}{b_{2}} \nu\left(x_{0} T_{\tau}-\Xi_{\varepsilon}^{\mathrm{PS}}\left(x_{0}\right)\right) \cdot \mu_{x_{0}}^{\mathrm{PS}}\left(x_{0} N_{\tau}\right)
$$

and hence

$$
\varepsilon \cdot b_{1}^{-1} \nu\left(x_{0} T_{\tau}\right) \cdot \mu_{x_{0}}^{\mathrm{PS}}\left(x_{0} N_{\tau}\right) \geq \frac{1}{b_{2}} \nu\left(x_{0} T_{\tau}-\Xi_{\varepsilon}^{\mathrm{PS}}\left(x_{0}\right)\right) \cdot \mu_{x_{0}}^{\mathrm{PS}}\left(x_{0} N_{\tau}\right) .
$$

Therefore

$$
\nu\left(x_{0} T_{\tau}-\Xi_{\varepsilon}^{\mathrm{PS}}\left(x_{0}\right)\right) \leq \varepsilon \cdot \frac{b_{2}}{b_{1}} \nu\left(x_{0} T_{\tau}\right)
$$

implying the claim.

By the $M$-invariance of $m^{\mathrm{BMS}}$, by Lemma 6.8

$$
\mu_{x m_{\theta}}^{\mathrm{PS}}\left(\Omega_{\varepsilon} \cap x m_{\theta} N_{\tau}\right)=\mu_{x m_{\theta}}^{\mathrm{PS}}\left(\Omega_{\varepsilon} \cap x N_{\tau} m_{\theta}\right)=\mu_{x}^{\mathrm{PS}}\left(\Omega_{\varepsilon} m_{\theta}^{-1} \cap x N_{\tau}\right) .
$$

Note that for any $x m_{\theta} \in \Xi_{\varepsilon}^{\mathrm{PS}}\left(x_{0}\right)$, we have

$$
\mu_{x}^{\mathrm{PS}}\left(x N_{\tau} \cap \Omega_{\varepsilon} m_{\theta}^{-1}\right)>(1-\varepsilon) \mu_{x}\left(x N_{\tau}\right) \geq \frac{\tau^{\delta}}{2 c_{0}} .
$$


By setting $O_{\theta}(x):=p_{\theta}\left(x N_{\rho} \cap \Omega_{\varepsilon} m_{\theta}^{-1}\right)$, we note that $O_{\theta}(x) U_{\theta}^{2 \tau}$ contains $x N_{\tau} \cap$ $\Omega_{\varepsilon} m_{\theta}^{-1}$ and hence for all small $0<\varepsilon \ll 1$,

$$
\mu_{x}^{\mathrm{PS}}\left(O_{\theta}(x) U_{\theta}^{2 \tau}\right) \geq(1-\varepsilon) \mu_{x}^{\mathrm{PS}}\left(x N_{\tau} \cap \Omega_{\varepsilon} m_{\theta}^{-1}\right) \geq \frac{\tau^{\delta}}{2 c_{0}} .
$$

Therefore the above three lemmas prove Theorem 6.12

\section{Window THEOREM FOR Hopf AVERAGE}

We will combine the results from previous sections and prove the window theorem 7.7 in this section. We first show that the disintegration along $U$ of $\lambda_{s}$, notation as in Section 3, has certain doubling properties, see Theorem 7.1. This is done by applying results in Section 5. in particular the key lemma, to $\lambda_{E, x, s}$ and the limiting measure $\mu_{x}^{\mathrm{PS}}$, in combination with Theorem 6.12, which gives a rather strong doubling property for the disintegration of the PS measure. As we mentioned in the introduction, in general, the weak* convergence of measures does not give control on the corresponding conditional measures, e.g., one should recall the wellknown discontinuity of the entropy. However, here the key lemma gives a good control both on the prelimiting measures $\lambda_{E, x, s}$ and the limit measure $\mu_{x}^{\mathrm{PS}}$, and helps us to draw some connection between the conditionals.

In order to obtain the Window Theorem 7.3, we flow by $a_{-s}$ for a suitably big $s$ and bring $[-T, T]$ to size $[-\rho, \rho]$. We are now working with $m_{E, s}^{\mathrm{BR}}$ rather than $\left.\frac{1}{m^{\mathrm{BR}}(E)} m^{\mathrm{BR}}\right|_{E}$, and the desired estimate follows from Theorem 7.1

7.1. Window theorem for $\chi_{E}$. Let $\Gamma$ be a convex cocompact subgroup with $\delta>1$. Let $E$ be a BMS box. For simplicity, we set

$$
\mu_{s}:=\mu_{E, s}^{\mathrm{BR}} \quad \text { and } \quad \lambda_{x, s}=\lambda_{E, x, s}
$$

defined in section 5. For $0<r \leq 1$ and $\rho>0$ as in (6.2), we put

$$
E_{s}(r):=\left\{x \in E a_{-s}: \frac{\left(\mu_{s}\right)_{x}^{U}[-2 \rho r, 2 \rho r]}{\left(\mu_{s}\right)_{x}^{U}[-2 \rho, 2 \rho]}>1-r\right\} .
$$

Theorem 7.1. There exist $0<r_{0}<1$ and $s_{0}>1$ (depending on $E$ ) such that for all $s>s_{0}$, we have

$$
\mu_{E, s}^{\mathrm{BR}}\left(E_{s}\left(r_{0}\right)\right)<1-r_{0} .
$$

Proof. Suppose not; then there are a subsequence $r_{i} \rightarrow 0$ and a subsequence $s_{i} \rightarrow$ $+\infty$ such that $\mu_{E, s_{i}}^{\mathrm{BR}}\left(E_{s_{i}}\left(r_{i}\right)\right) \geq 1-r_{i}$. Set

$$
\mu_{i}=\mu_{E, s_{i}}^{\mathrm{BR}} \quad \text { and } \quad E_{i}:=E_{s_{i}}\left(r_{i}\right) .
$$

Fix $\varepsilon>0$. Let $x_{0} \in \Omega_{\varepsilon}^{\prime}, 0<\tau<\rho, c_{0}>1$ and $\Xi_{\varepsilon}^{\mathrm{PS}}\left(x_{0}\right)$ be as in Theorem 6.12. Set $q_{0}:=m^{\mathrm{BMS}}\left(B_{x_{0}}(\tau)\right)>0$ and

$$
x_{0} T_{i}:=\left\{y \in x_{0} T_{\tau}: \lambda_{s_{i}, y}\left(E_{i} \cap y N_{\tau}\right)>\left(1-\sqrt{\frac{2 r_{i}}{q_{0}}}\right) \lambda_{s_{i}, y}\left(y N_{\tau}\right)\right\} .
$$

Recall the measure $\nu=\nu_{x_{0} T_{\rho}}$ from Theorem 6.12. We claim that for all large $i \gg 1$, we have

$$
\nu\left(x_{0} T_{i}\right) \geq\left(1-4 \sqrt{r_{i}}\right) \nu\left(x_{0} T_{\tau}\right) .
$$


We will first show that for all large $i \gg 1$,

$$
\mu_{i}\left(E_{i} \cap B_{x_{0}}(\tau)\right) \geq\left(1-\frac{2 r_{i}}{q_{0}}\right) \mu_{i}\left(B_{x_{0}}(\tau)\right) .
$$

If this does not hold, by passing to a subsequence, we have that

$$
\begin{aligned}
1-r_{i}<\mu_{i}\left(E_{i}\right) & =\mu_{i}\left(E_{i} \cap B_{x_{0}}(\tau)\right)+\mu_{i}\left(E_{i}-B_{x_{0}}(\tau)\right) \\
& \leq\left|\mu_{i}\right|-\frac{2 r_{i}}{q_{0}} \mu_{i}\left(B_{x_{0}}(\tau)\right) .
\end{aligned}
$$

Since $\left|\mu_{i}\right|=1$, it follows that

$$
\frac{2}{q_{0}} \mu_{i}\left(B_{x_{0}}(\tau)\right) \leq 1
$$

On the other hand, since $\mu_{i}$ weakly converges to $m^{\text {BMS }}$ by Theorem 3.1, we have

$$
\frac{\mu_{i}\left(B_{x_{0}}(\tau)\right)}{q_{0}} \rightarrow 1
$$

which gives a contradiction. This shows (7.2). Now, by the same type of argument as the proof of Lemma 6.15, we can show (7.2) implies (7.1).

Passing to a subsequence, which we continue to denote by $r_{i}$, we assume that $4 \sum_{i} \sqrt{r}_{i}<\varepsilon / 2$ and $2 r_{i} / q_{0}<\varepsilon$ for all $i$. If we set

$$
\Xi^{*}\left(x_{0}\right):=\cap_{i} x_{0} T_{i},
$$

then it follows that

$$
\nu\left(\Xi^{*}\left(x_{0}\right)\right)>(1-\varepsilon) \nu\left(x_{0} T_{\tau}\right) .
$$

Hence for all sufficiently small $\varepsilon>0$,

$$
\nu\left(\Xi_{\varepsilon}^{\mathrm{PS}}\left(x_{0}\right) \cap \Xi^{*}\left(x_{0}\right)\right)>\left(1-\left(1+c_{0}^{4}\right) \varepsilon\right) \nu\left(x_{0} T_{\tau}\right)>0 .
$$

Let $\Theta_{\varepsilon}(x)$ be given as in the Key Lemma 5.11 for $\{\rho, \tau\}$ applied to the set $E$ and the sequence $s_{i}$. Since $\operatorname{supp}(\nu) \subset\left\{x \in X: x^{-} \in \Lambda(\Gamma)\right\}$, we can find $x m_{\theta} \in \Xi^{*}\left(x_{0}\right) \cap \Xi_{\varepsilon}^{\mathrm{PS}}\left(x_{0}\right)$ (depending on $\varepsilon>0$ ) with $\left(x m_{\theta}\right)^{-}=x^{-} \in \Lambda(\Gamma)$ and $\theta \in \Theta_{\varepsilon}(x)$. Since $x^{+}=x_{0}^{+}$, we have $x^{ \pm} \in \Lambda(\Gamma)$.

By the $M$-invariance of the measure $\mu_{s_{i}}$ and as $x m_{\theta} \in x_{0} T_{i}$, we have

$$
\begin{aligned}
\lambda_{x, s_{i}}\left(E_{i} m_{\theta}^{-1} \cap x N_{\tau}\right) & =\lambda_{x m_{\theta}, s_{i}}\left(E_{i} \cap x m_{\theta} N_{\tau}\right) \\
& \geq\left(1-\sqrt{\frac{2 r_{i}}{q_{0}}}\right) \lambda_{x m_{\theta}, s_{i}}\left(x m_{\theta} N_{\tau}\right) \\
& =\left(1-\sqrt{\frac{2 r_{i}}{q_{0}}}\right) \lambda_{x, s_{i}}\left(x N_{\tau}\right),
\end{aligned}
$$

and hence

$$
\lambda_{x, s_{i}}\left(x N_{\tau}-E_{i} m_{\theta}^{-1}\right) \rightarrow 0 .
$$

Let $\left\{s_{j_{i}}\right\}$ be the corresponding subsequence given by Lemma 5.11 depending on $(x, \theta)$. By passing to that subsequence, we set $s_{i}:=s_{j_{i}}$.

For $O_{\theta}(x)$ as in Theorem 6.12 we consider $L_{i}:=p_{\theta}\left(O_{\theta}(x) U_{\theta}^{\tau} \cap E_{i} m_{\theta}^{-1}\right)$. By Lemma 7.2 below,

$$
\lambda_{x, s_{i}}\left(L_{i} U_{\theta}^{\rho}\right) \leq \frac{1}{1-r_{i}} \lambda_{x, s_{i}}\left(L_{i} U_{\theta}^{2 \tau}\right)
$$


Since $L_{i}=p_{\theta}\left(E_{i} m_{\theta}^{-1}\right) \cap O_{\theta}(x)$ and $\left\{t \in O_{\theta}(x): D\left(\sigma_{x, \theta}^{\tau}, t\right)=0\right\}=\emptyset$, it follows from the key Lemma 5.12 and (7.3) that for all large $i \gg 1$,

$$
\begin{aligned}
\lambda_{x, s_{i}}\left(O_{\theta}(x) U_{\theta}^{\rho}\right) & =\sigma_{x, \theta, s_{i}}^{\rho}\left(O_{\theta}(x)\right) \\
& \leq(1+\varepsilon) \sigma_{x, \theta, s_{i}}^{\rho}\left(L_{i}\right)=(1+\varepsilon) \lambda_{x, s_{i}}\left(x L_{i} U_{\theta}^{\rho}\right) .
\end{aligned}
$$

Therefore, we have

$$
\begin{aligned}
\lambda_{x, s_{i}}\left(O_{\theta}(x) U_{\theta}^{\rho}\right) & \leq \frac{(1+\varepsilon)}{\left(1-r_{i}\right)} \lambda_{x, s_{i}}\left(O_{\theta}(x) U_{\theta}^{2 \tau}\right) \\
& \leq(1+2 \varepsilon) \lambda_{x, s_{i}}\left(O_{\theta}(x) U_{\theta}^{2 \tau}\right) .
\end{aligned}
$$

Recall we chose $s_{i}=s_{j_{i}}$ so that Theorem 5.11holds for $\{\rho, \tau\}$. Hence, by sending $s_{i} \rightarrow \infty$, the above implies

$$
\mu_{x}^{\mathrm{PS}}\left(O_{\theta}(x) U_{\theta}^{\rho}\right) \leq(1+2 \varepsilon) \mu_{x}^{\mathrm{PS}}\left(O_{\theta}(x) U_{\theta}^{2 \tau}\right) .
$$

Together with Theorem 6.12, this gives

$$
2 \varepsilon \cdot \mu_{x}^{\mathrm{PS}}\left(O_{\theta}(x) U_{\theta}^{2 \tau}\right) \geq \mu_{x}^{\mathrm{PS}}\left(O_{\theta}(x) U_{\theta}^{2 \tau}\right),
$$

however, $\mu_{x}^{\mathrm{PS}}\left(O_{\theta}(x) U_{\theta}^{2 \tau}\right) \geq \frac{\tau^{\delta}}{8 c_{0}}>0$. This gives a contradiction and finishes the proof of Theorem 7.1 .

Lemma 7.2. Let $x m_{\theta} \in x_{0} T_{\tau}$ for $x_{0} \in \Omega$ and $s>0$. For any Borel subset $L_{\theta}(x) \subset p_{\theta}\left(x N_{\tau} \cap E_{s}(r) m_{\theta}^{-1}\right)$ and for all sufficiently small $0<r \ll 1$,

$$
\lambda_{x, s}\left(L_{\theta}(x) U_{\theta}^{\rho}\right) \leq \frac{1}{1-r} \lambda_{x, s}\left(L_{\theta}(x) U_{\theta}^{2 \tau}\right) .
$$

Proof. Set

$$
\tilde{L}=p_{\theta}\left(x N_{\tau} \cap E_{s}(r) m_{\theta}^{-1}\right) .
$$

Note that if $t \in \tilde{L}$ and hence $t \in p_{\theta}\left(E_{s}(r) m_{\theta}^{-1}\right)$, then $t m_{\theta}=z_{t} u_{0}$ for some $z_{t} \in E_{s}(r)$ and $u_{0} \in U^{\tau}$. On the other hand, it follows from the definition of $E_{s}(r)$ that

$$
\left|\left(\mu_{s}\right)_{z_{t}}^{U^{2 \rho}}\right| \leq \frac{1}{1-r}\left|\left(\mu_{s}\right)_{z_{t}}^{U^{2 r \rho}}\right|
$$

and hence

$$
\left|\left(\mu_{s}\right)_{t m_{\theta}}^{U^{\rho}}\right| \leq\left|\left(\mu_{s}\right)_{z_{t}}^{U^{\rho+\tau}}\right| \leq\left|\left(\mu_{s}\right)_{z_{t}}^{U^{2 \rho}}\right| \leq \frac{1}{1-r}\left|\left(\mu_{s}\right)_{z_{t}}^{U^{2 r \rho}}\right| \leq \frac{1}{1-r}\left|\left(\mu_{s}\right)_{t m_{\theta}}^{U^{2 r \rho+\tau}}\right| .
$$

Therefore, since $\mu_{E, s}^{\mathrm{BR}}$ is $M$-invariant, by Lemma 6.8

$$
\begin{aligned}
\lambda_{x, s}\left(L_{\theta}(x) U_{\theta}^{\rho}\right) & =\int_{t \in L_{\theta}(x)}\left|\left(\mu_{s}\right)_{t}^{U_{\theta}^{\rho}}\right| d \sigma_{s, x, \theta}^{\rho}(t) \\
& =\int_{t \in L_{\theta}(x)}\left|\left(\mu_{s}\right)_{t m_{\theta}}^{U^{\rho}}\right| d \sigma_{x, \theta, s}^{\rho}(t) \\
& \leq \frac{1}{1-r} \int_{t \in L_{\theta}(x)}\left|\left(\mu_{s}\right)_{t m_{\theta}}^{U^{2 \tau}}\right| d \sigma_{x, \theta, s}^{\rho}(t) \\
& =\frac{1}{1-r} \int_{t \in L_{\theta}(x)}\left|\left(\mu_{s}\right)_{t}^{U_{\theta}^{2 \tau}}\right| d \sigma_{x, \theta, s}^{\rho}(t) \\
& =\frac{1}{1-r} \lambda_{x, s}\left(L_{\theta}(x) U_{\theta}^{2 \tau}\right) .
\end{aligned}
$$


We deduce the following from Theorem 7.1 .

Theorem 7.3. There exists $0<r<1$ and $T_{0}>1$, depending on $E$, such that for all $T>T_{0}$

$$
m^{\mathrm{BR}}\left\{x \in E: \int_{-r T}^{r T} \chi_{E}\left(x u_{t}\right) d t<(1-r) \cdot \int_{-T}^{T} \chi_{E}\left(x u_{t}\right) d t\right\} \geq r \cdot m^{\mathrm{BR}}(E) .
$$

Proof. Setting

$$
E(s, r):=\left\{x \in E: \frac{\int_{-2 r \rho e^{s}}^{2 r \rho e^{s}} \chi_{E}\left(x u_{t}\right) d t}{\int_{-2 \rho e^{s}}^{2 \rho e^{s}} \chi_{E}\left(x u_{t}\right) d t} \geq 1-r\right\},
$$

it suffices to prove that for some $0<r<1$ and for all $s$ large.

$$
m^{\mathrm{BR}}(E(s, r))<(1-r) m^{\mathrm{BR}}(E) .
$$

We note that

$$
E(s, r)=\left\{x \in E: \frac{\left(\mu_{0, E}^{\mathrm{BR}}\right)_{x}^{U}\left[-2 r \rho e^{s}, 2 r \rho e^{s}\right]}{\left(\mu_{0, E}^{\mathrm{BR}}\right)_{x}^{U}\left[-2 \rho e^{s}, 2 \rho e^{s}\right]} \geq 1-r\right\},
$$

where $\left(\mu_{E, 0}^{\mathrm{BR}}\right)_{x}^{U}$ denotes the leafwise measure of $\mu_{E, 0}^{\mathrm{BR}}=\frac{\left.m^{\mathrm{BR}}\right|_{E}}{m^{\mathrm{BR}}(E)}$.

Note that (7.7) follows from Theorem 7.1 if we show

$$
m^{\mathrm{BR}}(E(s, r))=m^{\mathrm{BR}}(E) \cdot \mu_{E, s}^{\mathrm{BR}}\left(E_{s}(r)\right) \text { for all } 0<r<1 .
$$

We now show the above identity. Let $s$ be fixed. Then for BR a.e. points $x$, we have

$$
\begin{array}{rlr}
\frac{\left(\mu_{E, 0}^{\mathrm{BR}}\right)_{x}^{U}\left[-2 r \rho e^{s}, 2 r \rho e^{s}\right]}{\left(\mu_{E, 0}^{\mathrm{BR}}\right)_{x}^{U}\left[-2 \rho e^{s}, 2 \rho e^{s}\right]} & =\frac{\int_{-2 r \rho}^{2 r \rho} \chi_{E}\left(x a_{-s} u_{t} a_{s}\right) d t}{\int_{-2 \rho}^{2 \rho} \chi_{E}\left(x a_{-s} u_{t} a_{s}\right) d t} \quad \text { since } m^{\mathrm{BR}} \text { is } U \text {-invariant } \\
& =\frac{\int_{-2 r \rho}^{2 r \rho} \chi_{E a_{-s}}\left(y u_{t}\right) d t}{\int_{-2 \rho}^{2 \rho} \chi_{E a_{-s}}\left(y u_{t}\right) d t} \quad y=x a_{-s} \\
& =\frac{\left(\mu_{E a_{-s}, 0}^{\mathrm{BR}}\right)_{y}^{U}[-2 r \rho, 2 r \rho]}{\left(\mu_{E a_{-s}, 0}^{\mathrm{BR}}\right)_{y}^{U}[-2 \rho, 2 \rho]} & \text { since } m^{\mathrm{BR}} \text { is } U \text {-invariant } \\
& =\frac{\left(e^{(\delta-2) s} \mu_{E, s}^{\mathrm{BR}}\right)_{y}^{U}[-2 r \rho, 2 r \rho]}{\left(e^{(\delta-2) s} \mu_{E, s}^{\mathrm{BR}}\right)_{y}^{U}[-2 \rho, 2 \rho]} & \text { by the definition of } m_{E, s}^{\mathrm{BR}}
\end{array}
$$

Hence $a_{-s} E(s, r)$ coincides with $E_{s}(r)$, up to a BR null set. This implies the claim using the definition of $\mu_{E, s}^{\mathrm{BR}}$.

7.2. Ergodic decomposition and the Hopf ratio theorem. In this subsection, let $\mu$ be a locally finite $U$-invariant conservative measure on $X$. Let $\mathcal{M}_{\infty}(X)$ denote the space of locally finite measures on $X$ with weak* topology.

Let $\mathcal{A}$ denote a countably generated $\sigma$-algebra equivalent to the $\sigma$-algebra of all $U$-invariant subsets of $X$. There exist a $\mathcal{A}$-measurable conull set $X^{\prime}$ of $X$, a family $\left\{\mu_{x}=\mu_{x}^{\mathcal{A}}: x \in X^{\prime}\right\}$ of conditional measures on $X$ and a probability measure $\mu_{*}$ on $X$ which give rise to the ergodic decomposition of $\mu$ :

$$
\mu=\int \mu_{x} d \mu_{*}(x)
$$


where the map $X^{\prime} \rightarrow \mathcal{M}_{\infty}(X), x \mapsto \mu_{x}$, is Borel measurable, $\mu_{x}$ is a $U$-invariant, ergodic and conservative measure on $X$ and for any $\psi \in L^{1}(X, \mu)$,

$$
\mu(\psi)=\int_{x \in X} \mu_{x}(\psi) d \mu_{*}(x)
$$

see [7, 5.1.4].

The following is the Hopf ratio theorem in a form convenient for us ([1], see also 38).

Theorem 7.4. Let $\psi, \phi \in L^{1}\left(m^{\mathrm{BR}}\right)$ with $\phi \geq 0$. Furthermore suppose that $\psi$ and $\phi$ are compactly supported. Then

$$
\lim _{T} \frac{\int_{0}^{T} \psi\left(x u_{t}\right) d t}{\int_{0}^{T} \phi\left(x u_{t}\right) d t}=\lim _{T} \frac{\int_{-T}^{0} \psi\left(x u_{t}\right) d t}{\int_{-T}^{0} \phi\left(x u_{t}\right) d t}=\frac{\mu_{x}(\psi)}{\mu_{x}(\phi)}
$$

for $\mu$-a.e. $x \in\left\{x \in X: \sup _{T} \int_{0}^{T} \phi\left(x u_{t}\right) d t>0\right\} 2$

Lemma 7.5. Fix a compact subset $E \subset X$ with $\mu(E)>0$. Let $\phi$ be a non-negative compactly supported Borel function on $X$ such that $\left.\phi\right|_{E}>0$. For any $\rho>0$ there exists a compact subset $E_{\rho}(\phi) \subset E$ with $\mu\left(E-E_{\rho}(\phi)\right)<\rho \cdot \mu(E)$ satisfying:

(1) the map $x \mapsto \mu_{x}$ is continuous for all $x \in E_{\rho}(\phi)$;

(2) $\inf _{x \in E_{\rho}} \mu_{x}(\phi)>0$;

(3) for any $\psi \in C_{c}(X)$ the convergence

$$
\frac{\int_{0}^{T} \psi\left(x u_{t}\right) d t}{\int_{0}^{T} \phi\left(x u_{t}\right) d t} \rightarrow \frac{\mu_{x}(\psi)}{\mu_{x}(\phi)}
$$

is uniform on $E_{\rho}(\phi)$.

Proof. By Lusin's theorem, there exists a compact subset $E^{\prime} \subset E$ with $\mu\left(E-E^{\prime}\right)<$ $\frac{\rho}{3} \mu(E)$ and the map $x \mapsto \mu_{x}$ is continuous on $E^{\prime}$. Since $\int_{0}^{T} \phi\left(x u_{t}\right) d t \rightarrow+\infty$ for a.e. $x \in E$ by the conservativity of $\mu$, we have $\mu_{x}(\phi)>0$ almost all $x \in E$. Since $x \mapsto \mu_{x}(\phi)$ is a measurable map, it follows again by Lusin's theorem that there exists a compact subset $E^{\prime \prime} \subset E^{\prime}$ with $\mu\left(E^{\prime}-E^{\prime \prime}\right)<\frac{\rho}{3} \mu\left(E^{\prime}\right), \int_{0}^{\infty} \phi\left(x u_{t}\right) d t=\infty$ for all $x \in E^{\prime \prime}$, and $\inf _{x \in E^{\prime \prime}} \mu_{x}(\phi)>0$.

We claim that for any $\varepsilon>0$ and any compact subset $Q$ of $X$, there exists a compact subset $E_{0}=E_{0}(Q, \varepsilon) \subset E^{\prime \prime}$ such that $\mu\left(E^{\prime \prime}-E_{0}\right)<\varepsilon \mu\left(E^{\prime \prime}\right)$ and for all $\psi \in C(Q)$, the convergence

$$
\frac{\int_{0}^{T} \psi\left(x u_{t}\right) d t}{\int_{0}^{T} \phi\left(x u_{t}\right) d t} \rightarrow \frac{\mu_{x}(\psi)}{\mu_{x}(\phi)}
$$

is uniform on $E_{0}$. Let $\mathcal{B}=\left\{\psi_{j}\right\}$ be a countable dense subset of $C(Q)$ which includes the constant function $\chi_{Q}$. We can deduce from the Hopf ratio Theorem 7.4 and Egorov's theorem that there is a compact subset $E_{1} \subset E^{\prime \prime}$ such that $\mu\left(E^{\prime \prime}-E_{1}\right)<$ $\frac{\varepsilon}{3} \mu\left(E^{\prime \prime}\right), \sup _{x \in E_{1}} \mu_{x}(Q)<\infty$, and for each $\psi_{j} \in \mathcal{B}$, the convergence

$$
\frac{\int_{0}^{T} \psi_{j}\left(x u_{t}\right) d t}{\int_{0}^{T} \phi\left(x u_{t}\right) d t} \rightarrow \frac{\mu_{x}\left(\psi_{j}\right)}{\mu_{x}(\phi)}
$$

\footnotetext{
${ }^{2}$ note that for a.e. $x$ in this set $\mu_{x}(\phi)>0$, see [38, Page 3].
} 
is uniform on $E_{1}$. We will show the uniform convergence in $E_{1}$ for all $\psi \in C(Q)$. For any $\eta>0$, there exists $\psi_{j} \in \mathcal{B}$ such that $\left\|\psi_{j}-\psi\right\|_{\infty}<\eta$. Let $T_{0} \gg 1$ be such that

$$
\left|\frac{\int_{0}^{T} \psi_{j}\left(x u_{t}\right) d t}{\int_{0}^{T} \phi\left(x u_{t}\right) d t}-\frac{\mu_{x}\left(\psi_{j}\right)}{\mu_{x}(\phi)}\right| \leq \eta, \quad\left|\frac{\int_{0}^{T} \chi_{Q}\left(x u_{t}\right) d t}{\int_{0}^{T} \phi\left(x u_{t}\right) d t}-\frac{\mu_{x}\left(\chi_{Q}\right)}{\mu_{x}(\phi)}\right| \leq \eta
$$

for all $x \in E_{1}$ and $T \geq T_{0}$. Now for any $x \in E_{1}$ and $T \geq T_{0}$, we have

$$
\begin{aligned}
\left|\frac{\int_{0}^{T} \psi\left(x u_{t}\right) d t}{\int_{0}^{T} \phi\left(x u_{t}\right) d t}-\frac{\mu_{x}(\psi)}{\mu_{x}(\phi)}\right| \leq & \frac{\int_{0}^{T}\left|\psi\left(x u_{t}\right)-\psi_{j_{0}}\left(x u_{t}\right)\right| d t}{\int_{0}^{T} \phi\left(x u_{t}\right) d t} \\
& +\left|\frac{\int_{0}^{T} \psi_{j_{0}}\left(x u_{t}\right) d t}{\int_{0}^{T} \phi\left(x u_{t}\right) d t}-\frac{\mu_{x}\left(\psi_{j_{0}}\right)}{\mu_{x}(\phi)}\right|+\left|\frac{\mu_{x}\left(\psi_{j_{0}}\right)}{\mu_{x}(\phi)}-\frac{\mu_{x}(\psi)}{\mu_{x}(\phi)}\right| \\
& \leq \frac{\int_{0}^{T} \chi_{Q}\left(x u_{t}\right) d t}{\int_{0}^{T} \phi\left(x u_{t}\right) d t}\left\|\psi-\psi_{j}\right\|_{\infty}+\eta+\frac{\mu_{x}(Q)}{\mu_{x}(\phi)}\left\|\psi-\psi_{j}\right\|_{\infty} \\
& \leq \frac{\mu_{x}(Q)}{\mu_{x}(\phi)} \eta+\eta^{2}+\eta+\frac{\mu_{x}(Q)}{\mu_{x}(\phi)} \eta \\
& \leq \eta\left(2 a_{0}+\eta+1\right)
\end{aligned}
$$

where $a_{0}:=\sup _{x \in E_{1}} \frac{\mu_{x}(Q)}{\mu_{x}(\phi)}<\infty$. This proves the claim. Let $Q_{1} \subset Q_{2} \subset \cdots$ be an exhaustion of $X$ by compact sets. Then $E_{\rho}(\phi):=\cap_{i} E_{0}\left(Q_{i}, \frac{\rho}{4^{i+1}}\right)$ satisfies all the desired properties.

7.3. Window theorem for $\psi \in C_{c}(X)$ with $\left.\psi\right|_{E}>0$. Let $\Gamma$ be a convex cocompact subgroup with $\delta>1$. Let $\mathcal{A}$ denote a countably generated $\sigma$-algebra which is equivalent to the $\sigma$-algebra of all $U$-invariant subsets of $X$, as before.

Since $m^{\mathrm{BR}}$ is $U$-conservative by Theorem 6.7, we may write an ergodic decomposition

$$
m^{\mathrm{BR}}=\int_{x \in X^{\prime}} \mu_{x} d m_{*}^{\mathrm{BR}}(x)
$$

where $X^{\prime}$ is a $\mathcal{A}$-measurable conull set of $X, m_{*}^{\mathrm{BR}}$ is a probability measure on $X$, and for all $x \in X^{\prime}, \mu_{x}=\mu_{x}^{\mathcal{A}}$ is a $U$-invariant ergodic conservative measure.

Lemma 7.6. Let $E$ and $0<r<1$ be as in Theorem 7.3 , Let $\psi \in C_{c}(X)$ with $\left.\psi\right|_{E}>0$. For any $\rho>0$, there exists $s_{0} \geq 1$ such that for all $s>s_{0}$,

$$
m^{\mathrm{BR}}\left\{x \in E: \int_{-r s}^{r s} \psi\left(x u_{t}\right) d t \leq(1-r+\rho) \int_{-s}^{s} \psi\left(x u_{t}\right) d t\right\} \geq(r-2 \rho) \cdot m^{\mathrm{BR}}(E) .
$$

Proof. For simplicity, set

$$
F_{\rho}(s):=\left\{x \in E: \int_{-r s}^{r s} \psi\left(x u_{t}\right) d t \leq(1-r+\rho) \int_{-s}^{s} \psi\left(x u_{t}\right) d t\right\} .
$$

Let $E_{\rho}\left(\chi_{E}\right) \subset E$ be as in Lemma 7.5. Since $\left.\psi\right|_{E}>0$, there is a subset $E_{\rho}^{\prime}$ of $E_{\rho}\left(\chi_{E}\right)$ such that $m^{\mathrm{BR}}\left(E-E_{\rho}^{\prime}\right)<2 \rho \cdot m^{\mathrm{BR}}(E)$ and $\inf _{x \in E_{\rho}^{\prime}} \frac{\mu_{x}(\psi)}{\mu_{x}(E)}>0$. Then for all large $s$ (uniformly for all $x \in E_{\rho}\left(\chi_{E}\right)$ ),

$$
\int_{-s}^{s} \psi\left(x u_{t}\right) d t=\left(\frac{\mu_{x}(\psi)}{\mu_{x}(E)}+a_{x}(\psi, s)\right) \int_{-s}^{s} \chi_{E}\left(x u_{t}\right) d t
$$

where $\left|a_{x}(\psi, s)\right| \leq a(s) \rightarrow 0$ as $s \rightarrow \infty$ by Lemma 7.5 . 
Setting

$$
\tilde{E}(s, r)=\left\{x \in E: \int_{-r s}^{r s} \chi_{E}\left(x u_{t}\right) d t \geq(1-r) \cdot \int_{-s}^{s} \chi_{E}\left(x u_{t}\right) d t\right\},
$$

we claim that

$$
E_{\rho}^{\prime} \cap(E-\tilde{E}(s, r)) \subset F_{\rho}(s) \quad \text { for all large } s,
$$

from which the lemma follows by Theorem 7.3. For any $x \in E_{\rho}^{\prime} \cap(E-\tilde{E}(s, r))$,

$$
\begin{aligned}
\int_{-r s}^{r s} \psi\left(x u_{t}\right) d t & =\left(\frac{\mu_{x}(\psi)}{\mu_{x}(E)}+a_{x}(\psi, r s)\right) \int_{-r s}^{r s} \chi_{E}\left(x u_{t}\right) d t \\
& \leq\left(\frac{\mu_{x}(\psi)}{\mu_{x}(E)}+\left|a_{x}(\psi, r s)\right|\right)(1-r) \int_{-s}^{s} \chi_{E}\left(x u_{t}\right) d t \\
& \leq(1-r) \int_{-s}^{s} \psi\left(x u_{t}\right) d t+\left(\left|a_{x}(\psi, s)\right|+\left|a_{x}(\psi, r s)\right|\right)(1-r) \int_{-s}^{s} \chi_{E}\left(x u_{t}\right) .
\end{aligned}
$$

Let $s_{1}>1$ be such that for $s \geq s_{1}$ and for all $x \in E_{\rho}^{\prime}$,

$$
\frac{\left(\left|a_{x}(\psi, s)\right|+\left|a_{x}(\psi, r s)\right|\right)(1-r)}{\left|\frac{\mu_{x}(\psi)}{\mu_{x}(E)}+a_{x}(\psi, s)\right|} \leq \rho
$$

this is possible since $\frac{\mu_{x}(\psi)}{\mu_{x}(E)}$ is uniformly bounded from below by a positive number. Then the claim holds.

By taking $\rho=r / 4$ and replacing $3 r / 4$ by $r$ in the above lemma, we now obtain:

Theorem 7.7 (Window Theorem). Let $\psi \in C_{c}(X)$ be a non-negative function such that $\left.\psi\right|_{E}>0$. Then, there exists $0<r<1$ and $T_{0}>1$ such that for any $T \geq T_{0}$,

$$
m^{\mathrm{BR}}\left\{x \in E: \int_{-r T}^{r T} \psi\left(x u_{t}\right) d t<(1-r) \int_{-T}^{T} \psi\left(x u_{t}\right) d t\right\}>\frac{r}{2} \cdot m^{\mathrm{BR}}(E) .
$$

It is worth mentioning that $r$ obtained here may be rather small. The following lemma demonstrates how the window estimates for a sequence will be used.

Lemma 7.8. Let $\varepsilon>0$ and a sequence $s_{k} \rightarrow+\infty$ be given. Let $E$ and $\psi$ be as in Theorem 1.4. Fix $\rho>0$. Let $x_{k} \in E_{\rho}(\psi)$ be a sequence satisfying

$$
\int_{(1-\varepsilon) s_{k}}^{s_{k}} \psi\left(x_{k} u_{t}\right) d t \geq c \varepsilon \int_{0}^{s_{k}} \psi\left(x_{k} u_{t}\right) d t
$$

for some $c>0$ independent of $k$. Then for any $f \in C_{c}(X)$, as $k \rightarrow \infty$,

$$
\frac{\int_{(1-\varepsilon) s_{k}}^{s_{k}} f\left(x_{k} u_{t}\right) d t}{\int_{(1-\varepsilon) s_{k}}^{s_{k}} \psi\left(x_{k} u_{t}\right) d t} \sim \frac{\mu_{x_{k}}(f)}{\mu_{x_{k}}(\psi)} .
$$

Proof. By the Hopf ratio theorem, and Lemma 7.5, we have

$$
\int_{0}^{s} f\left(x_{k} u_{t}\right) d t=\frac{\mu_{x_{k}}(f)}{\mu_{x_{k}}(\psi)} \int_{0}^{s} \psi\left(x_{k} u_{t}\right) d t+a_{x_{k}}(s) \int_{0}^{s} \psi\left(x_{k} u_{t}\right) d t
$$


with $\lim _{s \rightarrow \infty} a_{x_{k}}(s)=0$, uniformly in $\left\{x_{k}\right\}$. Therefore

$$
\begin{aligned}
\int_{(1-\varepsilon) s_{k}}^{s_{k}} f\left(x_{k} u_{t}\right) d t= & \frac{\mu_{x_{k}}(f)}{\mu_{x_{k}}(\psi)} \int_{(1-\varepsilon) s_{k}}^{s_{k}} \psi\left(x_{k} u_{t}\right) d t \\
& +a_{x_{k}}\left(s_{k}\right) \int_{0}^{s_{k}} \psi\left(x_{k} u_{t}\right) d t-a_{x_{k}}\left((1-\varepsilon) s_{k}\right) \int_{0}^{(1-\varepsilon) s_{k}} \psi\left(x_{k} u_{t}\right) d t .
\end{aligned}
$$

Since

$$
\begin{aligned}
& \left|a_{x_{k}}\left(s_{k}\right) \int_{0}^{s_{k}} \psi\left(x_{k} u_{t}\right) d t-a_{x_{k}}\left((1-\varepsilon) s_{k}\right) \int_{0}^{(1-\varepsilon) s_{k}} \psi\left(x_{k} u_{t}\right) d t\right| \\
& \quad \leq\left|a_{x_{k}}\left(s_{k}\right)+a_{x_{k}}\left((1-\varepsilon) s_{k}\right)\right| \cdot \int_{0}^{s_{k}} \psi\left(x_{k} u_{t}\right) d t \\
& \quad \leq \frac{\left|a_{x_{k}}\left(s_{k}\right)+a_{x_{k}}\left((1-\varepsilon) s_{k}\right)\right| \int_{(1-\varepsilon) s_{k}}^{s_{k}} \psi\left(x_{k} u_{t}\right) d t}{c \cdot \varepsilon}
\end{aligned}
$$

we obtain that

$$
\frac{\int_{(1-\varepsilon) s_{k}}^{s_{k}} f\left(x_{k} u_{t}\right) d t}{\int_{(1-\varepsilon) s_{k}}^{s_{k}} \psi\left(x_{k} u_{t}\right) d t}=\frac{\mu_{x_{k}}(f)}{\mu_{x_{k}}(\psi)}+O\left(\frac{\left|a_{x_{k}}\left(s_{k}\right)+a_{x_{k}}\left((1-\varepsilon) s_{k}\right)\right|}{c \varepsilon}\right) .
$$

Since $a_{x_{k}}\left(s_{k}\right)+a_{x_{k}}\left((1-\varepsilon) s_{k}\right) \rightarrow 0$, uniformly in $\left\{x_{k}\right\}$, the lemma follows.

\section{Additional invariance And ERgodicity of BR For $\delta>1$}

Let $\Gamma$ be a convex cocompact subgroup with $\delta>1$.

8.1. Reduction. Let $\mathcal{A}, X^{\prime}$ and $m^{\mathrm{BR}}=\int_{x \in X} \mu_{x} d\left(m^{\mathrm{BR}}\right)_{*}(x)$ be the decomposition of $m^{\mathrm{BR}}$ into $U$-ergodic components, see Section 7.2 .

Our strategy in proving the $U$-ergodicity of $m^{\text {BR }}$ is to show that for a.e. $x \in X$, $\mu_{x}$ is $N$-invariant.

Fix a BMS box $E$ and a non-negative function $\psi \in C_{c}(X)$ with $\left.\psi\right|_{E}>0$. Let $0<r<1$ be as in the window theorem 7.7 and $r_{0}:=\frac{r}{16}$. Recall $E_{r_{0}}(\psi) \subset E$ from Lemma 7.5 .

The next subsection is devoted to a proof of the following:

Theorem 8.1. For any $x_{0} \in E_{r_{0}}(\psi) \cap \operatorname{supp}\left(m^{\mathrm{BR}}\right), \mu_{x_{0}}$ is $N$-invariant.

Lemma 8.2. There exists a BR-conull set $X^{\prime \prime}$ such that if $x, x n \in X^{\prime \prime}$ for $n \in N$, then $\mu_{x n}=n . \mu_{x}$.

Proof. Since $N$ is abelian and $U<N, n . \mu_{x}$ is $U$-invariant and ergodic for every $n \in$ $N$ and for a.e. $x$. Now since $m^{\mathrm{BR}}$ is $N$-invariant, we have $m^{\mathrm{BR}}=\int n \cdot \mu_{x} d\left(m^{\mathrm{BR}}\right)_{*}(x)$ is also a $U$-ergodic decomposition of $m^{\mathrm{BR}}$ for each $n \in N$. The claim now follows from the uniqueness of ergodic decomposition.

Corollary 8.3. $\mathrm{m}^{\mathrm{BR}}$ is $U$-ergodic.

Proof. Set

$$
F:=\left\{x \in X: \mu_{x} \text { is } N \text {-invariant }\right\} .
$$

By Lemma 8.2, the characteristic function $\chi_{F}$ is an $N$-invariant measurable function. Since $m^{\mathrm{BR}}$ is $N$-ergodic by Theorem 2.9 and $m^{\mathrm{BR}}(F)>0$ by Theorem 8.1 , it follows that $m^{\mathrm{BR}}(X-F)=0$. That is, $\mu_{x}=m^{\mathrm{BR}}$ for a.e. $x$, and since $\mu_{x}$ 's are $U$-ergodic components of $m^{\mathrm{BR}}$, the claim follows. 
8.2. Proof of Theorem 8.1. As we explained in the introduction, we will flow two nearby points in the generic set and study their divergence in the "intermediate range". We first need to prove a refinement of the window theorem, see Propositions 8.5 and 8.6 below.

$$
\text { Fix } x_{0} \in E_{r_{0}}(\psi) \cap \operatorname{supp}\left(m^{\mathrm{BR}}\right) .
$$

Proposition 8.4. There is a Borel subset $E^{\prime} \subset E$ such that $m^{\mathrm{BR}}\left(E-E^{\prime}\right)=0$ and for any $x \in E^{\prime}$ and all integers $m \geq 1$,

$$
x N \cap B\left(x_{0}, \frac{1}{m}\right) \cap E_{\rho}(\psi) \neq \emptyset .
$$

Proof. Set $N_{k}:=\left\{n_{z}:|z|<k\right\}$. Since $m^{\mathrm{BR}}$ is $N$-ergodic, by [10], there exists a full measure subset $E_{m}^{\prime}$ of $E$ such that for all $x \in E_{m}^{\prime}$

$$
\lim _{k} \frac{\int_{N_{k}} \chi_{B\left(x_{0}, 1 / m\right) \cap E_{r_{0}}(\psi)}\left(x n_{z}\right) d z}{\int_{N_{k}} \psi\left(x n_{z}\right) d z}=\frac{m^{\mathrm{BR}}\left(B\left(x_{0}, 1 / m\right) \cap E_{r_{0}}(\psi)\right)}{m^{\mathrm{BR}}(\psi)} .
$$

It suffices to take $E^{\prime}:=\cap_{m} E_{m}^{\prime}$.

Since $\inf _{x \in E_{r_{0}}(\psi)} \mu_{x}(\psi)>0$ and $x \mapsto \frac{1}{\mu_{x}(\psi)} \mu_{x}$ is continuous on $E_{r_{0}}(\psi)$, there exists a symmetric neighborhood $\mathcal{O}$ such that

$$
0<\inf _{g \in \mathcal{O}, x \in E_{r_{0}}(\psi)} \frac{\left|\mu_{x}(g \psi)\right|}{\mu_{x}(\psi)} \leq \sup _{g \in \mathcal{O}, x \in E_{r_{0}}(\psi)} \frac{\left|\mu_{x}(g \psi)\right|}{\mu_{x}(\psi)}<\infty .
$$

Set $K_{\psi}:=\operatorname{supp}(\psi) \mathcal{O}$ and $K_{\psi}^{\prime}:=\cap_{g \in \mathcal{O}} \operatorname{supp}(\psi) g$. By Theorem 7.7 for all $s \geq T_{0}$, the following set has BR measure at least $\frac{5 r}{16} m^{\mathrm{BR}}(E)$ :

$$
\begin{aligned}
& E_{s} \\
& :=\left\{x \in E_{r_{0}}(\psi) \cap E_{r_{0}}\left(\chi_{K_{\psi}}\right) \cap E_{r_{0}}\left(\chi_{K_{\psi}^{\prime}}\right): \int_{-r s}^{r s} \psi\left(x u_{t}\right) d t<(1-r) \int_{-s}^{s} \psi\left(x u_{t}\right) d t\right\} .
\end{aligned}
$$

Therefore, for each $s \geq T_{0}$, there exists a compact subset $\mathcal{G}(s)$ of $E_{s} \cap E^{\prime}$ with $m^{\mathrm{BR}}(\mathcal{G}(s))>\frac{r}{8} m^{\mathrm{BR}}(E)$.

We may write $\mathcal{G}(s)$ as $\mathcal{G}(s)_{+} \cup \mathcal{G}(s)_{\text {- where }}$

$$
\begin{aligned}
& \mathcal{G}(s)_{+}=\left\{x \in \mathcal{G}(s): \int_{0}^{r s} \psi\left(x u_{t}\right) d t \leq \frac{1-r}{2} \int_{0}^{s} \psi\left(x u_{t}\right) d t\right\} \\
& \mathcal{G}(s)_{-}=\left\{x \in \mathcal{G}(s): \int_{-r s}^{0} \psi\left(x u_{t}\right) d t \leq \frac{1-r}{2} \int_{-s}^{0} \psi\left(x u_{t}\right) d t\right\} .
\end{aligned}
$$

Therefore, there exists an infinite sequence $p_{i} \rightarrow+\infty$ such that $m^{\mathrm{BR}}\left(\mathcal{G}\left(p_{i}\right)_{+}\right) \geq \frac{r}{16}$ for all $i$ or $m^{\mathrm{BR}}\left(\mathcal{G}\left(p_{i}\right)_{-}\right) \geq \frac{r}{16}$ for all $i$.

In the following, we assume the former case that $m^{\mathrm{BR}}\left(\mathcal{G}\left(p_{i}\right)_{+}\right) \geq \frac{r}{16}$ for all $i$. The argument is symmetric in the other case.

Proposition 8.5. Fix integers $\ell, m>1$. There exists an infinite sequence $s_{k}=$ $s_{k}(\ell, m)$ and elements $x_{k}=x_{k}(\ell, m), y_{k}=y_{k}(\ell, m) \in \mathcal{G}\left(s_{k}\right)_{+}$which satisfy the following:

(1) $y_{k}=x_{k} \check{n}_{w_{k}}$ where $c_{1}^{-1} s_{k}^{-2} \ell^{-1} \leq\left|w_{k}\right| \leq c_{1} s_{k}^{-2} \ell^{-1}$ and $\left|\Im\left(w_{k}\right)\right| \geq \frac{\left|\Re\left(w_{k}\right)\right|}{c_{1}}$ where $c_{1}>1$ is independent of $\ell, \varepsilon, k$. 
(2) each $x_{k}$ satisfies

$$
\int_{(1-\varepsilon) s_{k}}^{s_{k}} \psi\left(x_{k} u_{t}\right) d t \geq \frac{r}{4 m} \int_{0}^{s_{k}} \psi\left(x_{k} u_{t}\right) d t .
$$

Proof. If $x \in \mathcal{G}_{+}(s)$, then, as $r<1$,

$$
\int_{r s}^{s} \psi\left(x u_{t}\right) \geq r \int_{0}^{s} \psi\left(x u_{t}\right) d t .
$$

By subdividing $[r, 1]$ into $m$ subintervals $I_{i}=\left(r+\frac{(j-1)}{m}, r+\frac{j}{m}\right)$ 's of length $\frac{1}{m}$, there exists an integer $1 \leq j=j(x, s) \leq m$ such that

$$
\int_{(r+(j-1) / m) s}^{(r+j / m) s} \psi\left(x u_{t}\right) d t \geq \frac{r}{4 m} \int_{0}^{s} \psi\left(x u_{t}\right) d t .
$$

Let $d_{0}=d_{0}(r / 16)>0$ be as in Proposition 4.4. Applying Proposition 4.4 to each $\mathcal{G}\left(p_{i}\right)_{+}$and a sequence $\left(p_{i} \ell\right)^{2}$, we can find $x_{i}, y_{i} \in \mathcal{G}\left(p_{i}\right)_{+}$satisfying $y_{i}=x_{i} \check{n}_{w_{i}}$ with $d_{0}^{-1} p_{i}^{-2} \ell^{-1} \leq\left|w_{i}\right| \leq d_{0} p_{i}^{-2} \ell^{-1}$ and $\left|\Im\left(w_{i}\right)\right| \geq \frac{\left|\Re w_{i}\right|}{d_{0}}$. Choose a subsequence $x_{i_{k}}$ of $\left\{x_{i}\right\}$ such that $j\left(x_{i_{k}}, p_{i_{k}}\right)$ is a constant, say, $j_{0}$. Setting $s_{k}:=\left(r+\frac{j_{0}}{m}\right) p_{i_{k}}$, $x_{k}:=x_{i_{k}}$ and $y_{k}=y_{i_{k}}$, we have

$$
\int_{(1-\varepsilon) s_{k}}^{s_{k}} \psi\left(x_{k} u_{t}\right) d t \geq \frac{r}{4 m} \int_{0}^{s_{k}} \psi\left(x_{k} u_{t}\right) d t
$$

and $r p_{i_{k}} \leq s_{k} \leq(r+1) p_{i_{k}}$. Hence, the claim follows with $c_{1}=d_{0}(r+1)^{2}$.

We now use the fact that the two orbits $x_{k} u_{t}$ and $y_{k} u_{t}$ stay "close" to each other, for all $t \in\left[0, s_{k}\right]$, to show that $y_{k}$ 's in Proposition 8.5 also satisfy the same type of window estimate. Let us fix some notation; writing $y_{k} u_{t}=x_{k} u_{t}\left(u_{-t} \check{n}_{w_{k}} u_{t}\right)$, we set

$$
p_{k}(t):=u_{-t} \check{n}_{w_{k}} u_{t}=\left(\begin{array}{cc}
1+t w_{k} & w_{k} \\
-t^{2} w_{k} & 1-t w_{k}
\end{array}\right),
$$

and $g_{k}=p_{k}\left(s_{k}\right)$.

Proposition 8.6. There are positive constants $c_{2}=c_{2}(\psi)$ and $\varepsilon_{0}=\varepsilon_{0}(\psi)$ such that for all $\varepsilon=\frac{1}{m}<\varepsilon_{0}$ and all $k \gg 1$,

$$
\int_{(1-\varepsilon) s_{k}}^{s_{k}} \psi\left(y_{k} u_{t}\right) d t \geq c_{2} \cdot \varepsilon \int_{0}^{s_{k}} \psi\left(y_{k} u_{t}\right) d t
$$

where $y_{k}=y_{k}(\ell, \varepsilon)$ is as in Proposition 8.5 .

Proof. There is a constant $c>0$ (independent of $\varepsilon$ ) such that $\left|p_{k}(t) g_{k}^{-1}\right|<c \varepsilon$ for all $t \in\left[(1-\varepsilon) s_{k}, s_{k}\right]$. Hence, for all $\ell \gg 1$ (independent of $\varepsilon$ ), we have $p_{k}(t) \in \mathcal{O}$ for all $t \in\left[0, s_{k}\right]$.

Claim (1): For some constant $b_{1}>0$, independent of $\varepsilon$, we have for all $k \gg 1$,

$$
\int_{0}^{s_{k}} \psi\left(x_{k} u_{t}\right) d t \geq b_{1} \int_{0}^{s_{k}} \psi\left(y_{k} u_{t}\right) d t
$$

By the definition of $K_{\psi}$ and $K_{\psi}^{\prime}$, since $y_{k} u_{t} \in x_{k} u_{t} \mathcal{O}$, we have $\chi_{K_{\psi}^{\prime}}\left(y_{k} u_{t}\right) \leq$ $\chi_{K_{\psi}}\left(x_{k} u_{t}\right)$ for all $t \in\left[0, s_{k}\right]$. In particular, we have

$$
\int_{0}^{s_{k}} \chi_{K_{\psi}}\left(x_{k} u_{t}\right) d t \geq \int_{0}^{s_{k}} \chi_{K_{\psi}^{\prime}}\left(y_{k} u_{t}\right) d t
$$


On the other hand, we have

$$
\begin{aligned}
& \int_{0}^{s_{k}} \psi\left(y_{k} u_{t}\right) d t=\frac{\mu_{y_{k}}(\psi)}{\mu_{y_{k}}\left(\chi_{K_{\psi}^{\prime}}\right)} \int_{0}^{s_{k}} \chi_{K_{\psi}^{\prime}}\left(y_{k} u_{t}\right) d t+a_{x_{k}}\left(\psi, s_{k}\right) \int_{0}^{s_{k}} \chi_{K_{\psi}^{\prime}}\left(x_{k} u_{t}\right) d t ; \\
& \int_{0}^{s_{k}} \psi\left(x_{k} u_{t}\right) d t=\frac{\mu_{x_{k}}(\psi)}{\mu_{x_{k}}\left(\chi_{K_{\psi}}\right)} \int_{0}^{s_{k}} \chi_{K_{\psi}}\left(x_{k} u_{t}\right) d t+a_{x_{k}}\left(\psi, s_{k}\right) \int_{0}^{s_{k}} \chi_{K_{\psi}}\left(x_{k} u_{t}\right) d t
\end{aligned}
$$

with $\max \left\{\left|a_{x_{k}}\left(\psi, s_{k}\right)\right|,\left|a_{y_{k}}\left(\psi, s_{k}\right)\right|\right\} \leq a\left(s_{k}\right) \rightarrow 0$ as $k \rightarrow \infty$.

As $\frac{\mu_{y_{k}}(\psi)}{\mu_{y_{k}}\left(\chi_{K_{\psi}^{\prime}}\right)}$ and $\frac{\mu_{x_{k}}(\psi)}{\mu_{x_{k}}\left(\chi_{K_{\psi}}\right)}$ are uniformly bounded from below and above, by the choice of $x_{k}$ and $y_{k}$, there exists $b>0$ such that for all large $k \gg 1$,

$$
\int_{0}^{s_{k}} \psi\left(x_{k} u_{t}\right) d t \geq b \int_{0}^{s_{k}} \chi_{K_{\psi}}\left(x_{k} u_{t}\right) d t \geq b \int_{0}^{s_{k}} \chi_{K_{\psi}^{\prime}}\left(y_{k} u_{t}\right) d t \geq b^{2} \int_{0}^{s_{k}} \psi\left(y_{k} u_{t}\right) d t
$$

finishing the proof of Claim (1).

Claim (2): For some constant $b_{2}>0$, independent of $\varepsilon$, we have for all $k \gg 1$,

$$
\int_{(1-\varepsilon) s_{k}}^{s_{k}} \chi_{K_{\psi}}\left(x_{k} u_{t}\right) d t \leq b_{2} \int_{(1-\varepsilon) s_{k}}^{s_{k}} \psi\left(x_{k} u_{t}\right) d t .
$$

By Lemma 7.8 and its proof, we have

$$
\begin{aligned}
\int_{(1-\varepsilon) s_{k}}^{s_{k}} \chi_{K_{\psi}}\left(x_{k} u_{t}\right) d t= & \frac{\mu_{x_{k}}\left(\chi_{K_{\psi}}\right)}{\mu_{x_{k}}(\psi)} \int_{(1-\varepsilon) s_{k}}^{s_{k}} \psi\left(x_{k} u_{t}\right) d t \\
& +\frac{4\left(a\left(s_{k}\right)+a\left(\varepsilon s_{k}\right)\right)}{r \varepsilon} \cdot \int_{(1-\varepsilon) s_{k}}^{s_{k}} \psi\left(x_{k} u_{t}\right) d t .
\end{aligned}
$$

Since $\frac{\mu_{x_{k}}(\psi)}{\mu_{x_{k}}\left(\chi_{K_{\psi}}\right)}$ is uniformly bounded from above and below by positive constants, it suffices to take $k$ large enough so that $\left(a\left(s_{k}\right)+a\left(\varepsilon s_{k}\right)\right) \leq \varepsilon$ to finish the proof of Claim (2).

We have

$$
\begin{aligned}
\int_{(1-\varepsilon) s_{k}}^{s_{k}} \psi\left(y_{k} u_{t}\right) d t & =\int_{(1-\varepsilon) s_{k}}^{s_{k}} \psi\left(x_{k} u_{t} p_{k}(t)\right) d t \\
& \geq \int_{(1-\varepsilon) s_{k}}^{s_{k}} \psi\left(x_{k} u_{t} g_{k}\right) d t-\int_{(1-\varepsilon) s_{k}}^{s_{k}}\left|\psi\left(x_{k} u_{t} p_{k}(t)\right)-\psi\left(x_{k} u_{t} g_{k}\right)\right| d t
\end{aligned}
$$

By (8.3), for all large $k$,

$$
\begin{aligned}
\int_{(1-\varepsilon) s_{k}}^{s_{k}}\left|\psi\left(x_{k} u_{t} p_{k}(t)\right)-\psi\left(x_{k} u_{t} g_{k}\right)\right| d t & \leq c_{\psi} \varepsilon \int_{(1-\varepsilon) s_{k}}^{s_{k}} \chi_{K_{\psi}}\left(x_{k} u_{t}\right) d t \\
& \leq c_{\psi} b_{2} \varepsilon \int_{(1-\varepsilon) s_{k}}^{s_{k}} \psi\left(x_{k} u_{t}\right)
\end{aligned}
$$

where $c_{\psi}$ is the Lipschitz constant of $\psi$. Since $g_{k} \in \mathcal{O}$ and hence $\frac{\mu_{x_{k}}\left(g_{k} \psi\right)}{\mu_{x_{k}}(\psi)}$ is uniformly bounded from above and below, we can deduce that for some $c>1$,

$$
c^{-1} \int_{(1-\varepsilon) s_{k}}^{s_{k}} \psi\left(x_{k} u_{t}\right) d t \leq \int_{(1-\varepsilon) s_{k}}^{s_{k}} \psi\left(x_{k} u_{t} g_{k}\right) d t \leq c \int_{(1-\varepsilon) s_{k}}^{s_{k}} \psi\left(x_{k} u_{t}\right) d t .
$$


Therefore, the above estimates together with (8.2) imply that for all $k$ large,

$$
\begin{aligned}
\int_{(1-\varepsilon) s_{k}}^{s_{k}} \psi\left(y_{k} u_{t}\right) d t & \geq\left(c^{-1}-c_{\psi} b_{2} \varepsilon\right) \int_{(1-\varepsilon) s_{k}}^{s_{k}} \psi\left(x_{k} u_{t}\right) d t \\
& \geq \frac{\left(c^{-1}-c_{\psi} b_{2} \varepsilon\right) r \varepsilon}{4} \int_{0}^{s_{k}} \psi\left(x_{k} u_{t}\right) d t \\
& \geq \frac{b_{1}\left(c^{-1}-c_{\psi} b_{2} \varepsilon\right) r \varepsilon}{4} \int_{0}^{s_{k}} \psi\left(y_{k} u_{t}\right) d t
\end{aligned}
$$

Now the proposition follows with $c_{2}=\frac{b_{1} r}{8 c}$ and $\varepsilon_{0}=\frac{1}{2 b_{2} c_{\psi} c}$.

We will now flow $x_{k}$ and $y_{k}$ for the period of time $\left[(1-\varepsilon) s_{k}, s_{k}\right]$. By the construction of these points, these two pieces of orbits are almost parallel and they essentially differ by $g_{k}$ which is of size $\mathrm{O}(1)$. More importantly these "short" pieces of the orbits already become equidistributed. This will show that some ergodic component is invariant by a nontrivial element in $N-U$ and the proof can be concluded from there using standard arguments.

Fix $\ell \in \mathbb{N}$. Let $\varepsilon_{i}=\frac{1}{i}>0$ for $i \in \mathbb{N}$. We choose $s_{k}\left(\varepsilon_{1}, \ell\right)$ and $x_{k}\left(\varepsilon_{1}, \ell\right), y_{k}\left(\varepsilon_{1}, \ell\right) \in$ $\mathcal{G}\left(s_{k}\left(\varepsilon_{1}, \ell\right)\right)_{+}$as in Proposition 8.5. Together with Proposition 8.6. there exists $\alpha_{1}>0$ independent of $\varepsilon_{1}$ and $k$ such that

$$
\begin{aligned}
& \int_{\left(1-\varepsilon_{1}\right) s_{k}\left(\varepsilon_{1}, \ell\right)}^{s_{k}\left(\varepsilon_{1}, \ell\right)} \psi\left(x_{k}\left(\varepsilon_{1}, \ell\right) u_{t}\right) d t \geq \alpha_{1} \varepsilon_{1} \int_{0}^{s_{k}\left(\varepsilon_{1}, \ell\right)} \psi\left(x_{k}\left(\varepsilon_{1}, \ell\right) u_{t}\right) d t \quad \text { and } \\
& \int_{\left(1-\varepsilon_{1}\right) s_{k}\left(\varepsilon_{1}, \ell\right)}^{s_{k}\left(\varepsilon_{1}, \ell\right)} \psi\left(y_{k}\left(\varepsilon_{1}, \ell\right) u_{t}\right) d t \geq \alpha_{1} \varepsilon_{1} \int_{0}^{s_{k}\left(\varepsilon_{1}, \ell\right)} \psi\left(y_{k}\left(\varepsilon_{1}, \ell\right) u_{t}\right) d t .
\end{aligned}
$$

By passing to a subsequence, we assume that $x_{k}\left(\varepsilon_{1}, \ell\right) \rightarrow x_{\varepsilon_{1}, \ell}$, and hence $y_{k}\left(\varepsilon_{1}, \ell\right) \rightarrow y_{\varepsilon_{1}, \ell}$, and $p_{k}\left(s_{k}\left(\varepsilon_{1}, \ell\right)\right)$ converges to $n_{v_{\varepsilon_{1}, \ell}}:=\left(\begin{array}{cc}1 & 0 \\ v_{\varepsilon_{1}, \ell} & 1\end{array}\right) \in N$ where $\frac{1}{c_{1} \ell} \leq\left|v_{\varepsilon_{1}, \ell}\right| \leq \frac{c_{1}}{\ell}$ and $\left|\Im\left(v_{\varepsilon_{1}, \ell}\right)\right| \geq \frac{\left|\Re\left(v_{\varepsilon_{1}, \ell}\right)\right|}{c_{1}}$.

We proceed by induction: by dividing the interval $\left[\left(1-\varepsilon_{i}\right) s_{k}\left(\varepsilon_{i}, \ell\right), s_{k}\left(\varepsilon_{i}, \ell\right)\right]$ into subintervals of length $\varepsilon_{i+1}$ as in the proof of Proposition 8.5 we can find a sequence $s_{k}\left(\varepsilon_{i+1}, \ell\right)$ and subsequences $x_{k}\left(\varepsilon_{i+1}, \ell\right)$ of $x_{k}\left(\varepsilon_{i}, \ell\right)$ and $y_{k}\left(\varepsilon_{i+1}, \ell\right)$ of $y_{k}\left(\varepsilon_{i}, \ell\right)$ satisfying

$$
\begin{aligned}
& \int_{\left(1-\varepsilon_{i+1}\right) s_{k}\left(\varepsilon_{i+1}, \ell\right)}^{s_{k}\left(\varepsilon_{i+1}, \ell\right)} \psi\left(x_{k}\left(\varepsilon_{i+1}, \ell\right) u_{t}\right) d t \geq \alpha_{1} \varepsilon_{i+1} \int_{0}^{s_{k}\left(\varepsilon_{i+1}, \ell\right)} \psi\left(x_{k}\left(\varepsilon_{i+1}, \ell\right) u_{t}\right) d t \\
& \int_{\left(1-\varepsilon_{i+1}\right) s_{k}\left(\varepsilon_{i+1}, \ell\right)}^{s_{k}\left(\varepsilon_{i+1}, \ell\right)} \psi\left(y_{k}\left(\varepsilon_{i+1}, \ell\right) u_{t}\right) d t \geq \alpha_{1} \varepsilon_{i+1} \int_{0}^{s_{k}\left(\varepsilon_{i+1}, \ell\right)} \psi\left(y_{k}\left(\varepsilon_{i+1}, \ell\right) u_{t}\right) d t
\end{aligned}
$$

and $p_{k}\left(s_{k}\left(\varepsilon_{i+1}, \ell\right)\right)$ converges to some element $n_{v_{\varepsilon_{i+1}, \ell}}:=\left(\begin{array}{cc}1 & 0 \\ v_{\varepsilon_{i+1}, \ell} & 1\end{array}\right) \in N$ where $\frac{1}{c_{1} \ell} \leq\left|v_{\varepsilon_{i+1}, \ell}\right| \leq \frac{c_{1}}{\ell}$ and $\left|\Im\left(v_{\varepsilon_{i+1}, \ell}\right)\right| \geq \frac{\left|\Re\left(v_{\varepsilon_{i+1}, \ell}\right)\right|}{c_{1}}$.

Clearly, as $i \rightarrow \infty$, we have $x_{k}\left(\varepsilon_{i}, \ell\right) \rightarrow x_{\varepsilon_{1}, \ell}$ and $y_{k}\left(\varepsilon_{i}, \ell\right) \rightarrow x_{\varepsilon_{1}, \ell}$. By passing to a subsequence, we may assume that $v_{\varepsilon_{i}, \ell}$ converges to an element $v_{\ell} \in N$. Note that $\frac{1}{c_{1} \ell} \leq\left|v_{\ell}\right| \leq \frac{c_{1}}{\ell}$ and $\left|\Im\left(v_{\ell}\right)\right| \geq \frac{\left|\Re\left(v_{\ell}\right)\right|}{c_{1}}$.

Let $\ell_{0}>1$ be large enough so that $n_{v_{\ell}} \in \mathcal{O}$ for all $\ell>\ell_{0}$. 
Proposition 8.7. Let $\ell>\ell_{0}$ and set $x_{\ell}:=x_{\varepsilon_{1}, \ell}$. For any $f \in C_{c}(X)$, we have

$$
\frac{\mu_{x_{\ell}}(f)}{\mu_{x_{\ell}}(\psi)}=\frac{\mu_{x_{\ell}}\left(n_{v_{\ell}} \cdot f\right)}{\mu_{x_{\ell}}\left(n_{v_{\ell}} \cdot \psi\right)}
$$

Proof. We claim that there exists a constant $b>0$ such that for each $i \geq 1$, the following holds for all $k \gg_{i} 1$ :

$$
\left|\frac{\mu_{y_{k}\left(\varepsilon_{i}, \ell\right)}(f)}{\mu_{y_{k}\left(\varepsilon_{i}, \ell\right)}(\psi)}-\frac{\mu_{x_{k}\left(\varepsilon_{i}, \ell\right)}\left(n_{v_{\varepsilon_{i}, \ell}} \cdot f\right)}{\mu_{x_{k}\left(\varepsilon_{i}, \ell\right)}\left(n_{\left.v_{\varepsilon_{i}, \ell} \cdot \psi\right)}\right.}\right|<b \varepsilon_{i} .
$$

We first deduce the proposition from this claim. Since both $y_{k}\left(\varepsilon_{i}, \ell\right), x_{k}\left(\varepsilon_{i}, \ell\right)$ belong to the set $E_{r / 16}(\psi)$ and converge to $x_{\ell}$, and $f, \psi \in C_{c}(X)$ have compact supports, $\mu_{y_{k}\left(\varepsilon_{i}, \ell\right)}(f) \rightarrow \mu_{x_{\ell}}(f)$ and $\mu_{y_{k}\left(\varepsilon_{i}, \ell\right)}(\psi) \rightarrow \mu_{x_{\ell}}(\psi)$ as $k \rightarrow \infty$.

Since $n_{v_{\varepsilon_{i}, \ell}} . f$ converges to $n_{v_{\ell}} . f$ pointwise as $i \rightarrow \infty$ and the supports of all functions involved are contained in one fixed compact subset of $X$, we have $\mu_{x_{k}\left(\varepsilon_{i}, \ell\right)}\left(n_{v_{\varepsilon_{i}, \ell}} . f\right) \rightarrow \mu_{x_{\ell}}\left(n_{v_{\varepsilon_{i}, \ell}} . f\right)$ as $k \rightarrow \infty$. Similarly, $\mu_{x_{k}\left(\varepsilon_{i}, \ell\right)}\left(n_{v_{\varepsilon_{i}, \ell} . \psi} \rightarrow\right.$ $\mu_{x_{\ell}}\left(n_{v_{\varepsilon_{i}, \ell}} . \psi\right)$ as $k \rightarrow \infty$. Hence (8.6) implies, by taking $k \rightarrow \infty$, that

$$
\left|\frac{\mu_{x_{\ell}}(f)}{\mu_{x_{\ell}}(\psi)}-\frac{\mu_{x_{\ell}}\left(n_{v_{\varepsilon_{i}, \ell}} \cdot f\right)}{\mu_{x_{\ell}}\left(n_{v_{\varepsilon_{i}, \ell}} \cdot \psi\right)}\right| \leq b \varepsilon_{i} .
$$

Now by taking $i \rightarrow \infty$, this proves the proposition as $\varepsilon_{i} \rightarrow 0$.

To prove Claim (8.6), fixing $\varepsilon:=\varepsilon_{i}$, we set $v=v_{\varepsilon_{i}}, s_{k}=s_{k}\left(\varepsilon_{i}\right), x_{k}=x_{k}\left(\varepsilon_{i}, \ell\right)$ and $y_{k}=y_{k}\left(\varepsilon_{i}, \ell\right)$ for simplicity. By Lemma 7.8, we have, as $k \rightarrow \infty$,

$$
\frac{\int_{(1-\varepsilon) s_{k}}^{s_{k}} f\left(y_{k} u_{t}\right) d t}{\int_{(1-\varepsilon) s_{k}}^{s_{k}} \psi\left(y_{k} u_{t}\right) d t} \sim \frac{\mu_{y_{k}}(f)}{\mu_{y_{k}}(\psi)} .
$$

Since $n_{v_{\ell}}^{+} \in \mathcal{O}$, similar calculation implies that, as $k \rightarrow \infty$,

$$
\frac{\int_{(1-\varepsilon) s_{k}}^{s_{k}} n_{v} \cdot f\left(x_{k} u_{t}\right) d t}{\int_{(1-\varepsilon) s_{k}}^{s_{k}} n_{v} \cdot \psi\left(x_{k} u_{t}\right) d t} \sim \frac{\mu_{x_{k}}\left(n_{v} \cdot f\right)}{\mu_{x_{k}}\left(n_{v} \cdot \psi\right)}
$$

Therefore the claim follows if we show for all large $k \gg_{i} 1$,

$$
\left|\frac{\int_{(1-\varepsilon) s_{k}}^{s_{k}} f\left(y_{k} u_{t}\right) d t}{\int_{(1-\varepsilon) s_{k}}^{s_{k}} \psi\left(y_{k} u_{t}\right) d t}-\frac{\int_{(1-\varepsilon) s_{k}}^{s_{k}} n_{v} \cdot f\left(x_{k} u_{t}\right) d t}{\int_{(1-\varepsilon) s_{k}}^{s_{k}} n_{v} \cdot \psi\left(x_{k} u_{t}\right) d t}\right| \leq b \varepsilon
$$

for some $b>0$ independent of $\varepsilon$. Let $c_{f}$ and $c_{\psi}$ denote the Lipschitz constants of $f$ and $\psi$ respectively. Hence for all $t \in\left[(1-\varepsilon) s_{k}, s_{k}\right]$ and large $k \gg 1$,

$$
\left|f\left(x_{k} u_{t} p_{k}(t)\right)-f\left(x_{k} u_{t} v_{\ell}\right)\right|<c_{f}\left(\varepsilon+s_{k}^{-1}\right) \leq 2 \varepsilon c_{f}
$$

and

$$
\left|\psi\left(x_{k} u_{t} p_{k}(t)\right)-\psi\left(x_{k} u_{t} v_{\ell}\right)\right|<c_{\psi}\left(\varepsilon+s_{k}^{-1}\right) \leq 2 \varepsilon c_{\psi}
$$


We have

$$
\begin{aligned}
\frac{\int_{(1-\varepsilon) s_{k}}^{s_{k}} f\left(y_{k} u_{t}\right) d t}{\int_{(1-\varepsilon) s_{k}}^{s_{k}} \psi\left(y_{k} u_{t}\right) d t}= & \frac{\int_{(1-\varepsilon) s_{k}}^{s_{k}} f\left(x_{k} u_{t} p_{k}(t)\right) d t}{\int_{(1-\varepsilon) s_{k}}^{s_{k}} \psi\left(x_{k} u_{t} p_{k}(t)\right) d t} \\
= & \frac{\int_{(1-\varepsilon) s_{k}}^{s_{k}} n_{v} \cdot f\left(x_{k} u_{t}\right) d t}{\int_{(1-\varepsilon) s_{k}}^{s_{k}} \psi\left(x_{k} u_{t} p_{k}(t)\right) d t} \\
& +\frac{\int_{(1-\varepsilon) s_{k}}^{s_{k}} f\left(x_{k} u_{t} p_{k}(t)\right)-n_{v} \cdot f\left(x_{k} u_{t}\right) d t}{\int_{(1-\varepsilon) s_{k}}^{s_{k}} \psi\left(x_{k} u_{t} p_{k}(t)\right) d t} .
\end{aligned}
$$

Let $K_{0}=K_{\psi} \cup K_{f}$. Then the above estimate, (8.5) and (8.8) with $f=\chi_{K_{0}}$, imply that

$$
\begin{aligned}
\left|\frac{\int_{(1-\varepsilon) s_{k}}^{s_{k}} f\left(x_{k} u_{t} p_{k}(t)\right)-n_{v} \cdot f\left(x_{k} u_{t}\right) d t}{\int_{(1-\varepsilon) s_{k}}^{s_{k}} \psi\left(x_{k} u_{t} p_{k}(t)\right) d t}\right| & \leq 2 c_{f} c \varepsilon \frac{\int_{(1-\varepsilon) s_{k}}^{s_{k}} \chi_{K_{0}}\left(x_{k} u_{t}\right) d t}{\int_{(1-\varepsilon) s_{k}}^{s_{k}} \psi\left(x_{k} u_{t}\right) d t} \\
& \leq 4 c_{f} c \varepsilon \frac{\mu_{x_{k}}\left(K_{0}\right)}{\mu_{x_{k}}(\psi)}
\end{aligned}
$$

On the other hand, we have

$$
\begin{aligned}
& \frac{\int_{(1-\varepsilon) s_{k}}^{s_{k}} n_{v} \cdot f\left(x_{k} u_{t}\right) d t}{\int_{(1-\varepsilon) s_{k}}^{s_{k}} \psi\left(x_{k} u_{t} p_{k}(t)\right) d t} \\
& \quad=\frac{\int_{(1-\varepsilon) s_{k}}^{s_{k}} n_{v} \cdot f\left(x_{k} u_{t}\right) d t}{\int_{(1-\varepsilon) s_{k}}^{s_{k}} n_{v} \cdot \psi\left(x_{k} u_{t}\right) d t} \cdot\left(1+\frac{\int_{(1-\varepsilon) s_{k}}^{s_{k}} \psi\left(x_{k} u_{t} p_{k}(t)\right)-n_{v} \cdot \psi\left(x_{k} u_{t}\right) d t}{\int_{(1-\varepsilon) s_{k}}^{s_{k}} n_{v} \cdot \psi\left(x_{k} u_{t}\right) d t}\right)^{-1} .
\end{aligned}
$$

Similar estimate as above gives

$$
\left|\frac{\int_{(1-\varepsilon) s_{k}}^{s_{k}} \psi\left(x_{k} u_{t} p_{k}(t)\right)-n_{v} \cdot \psi\left(x_{k} u_{t}\right) d t}{\int_{(1-\varepsilon) s_{k}}^{s_{k}} n_{v} \cdot \psi\left(x_{k} u_{t}\right) d t}\right| \leq 4 c_{\psi} c \varepsilon \frac{\mu_{x_{k}}\left(K_{0}\right)}{\mu_{x_{k}}(\psi)} .
$$

All these together imply there exists a constant $c^{\prime}>0$ (depending on $f$ and $\psi$ but independent of $\varepsilon$ ) such that

$$
\frac{\int_{(1-\varepsilon) s_{k}}^{s_{k}} f\left(y_{k} u_{t}\right) d t}{\int_{(1-\varepsilon) s_{k}}^{s_{k}} \psi\left(y_{k} u_{t}\right) d t}=\left(1+c^{\prime} \varepsilon\right) \frac{\int_{(1-\varepsilon) s_{k}}^{s_{k}} n_{v} \cdot f\left(x_{k} u_{t}\right) d t}{\int_{(1-\varepsilon) s_{k}}^{s_{k}} n_{v} \cdot \psi\left(x_{k} u_{t}\right) d t}+c^{\prime} \varepsilon .
$$

Now by (8.7), (8.8), this implies the claim (8.9).

The following proposition finishes the proof of Theorem 8.1 .

Proposition 8.8. $\mu_{x_{0}}$ is invariant under $N$.

Proof. The set $\left\{n \in N: n . \mu_{x_{0}}=\mu_{x_{0}}\right\}$ is a closed subgroup which contains $U$. Let $x_{\ell}$ and $v_{\ell}$ be as in Proposition 8.7. Since $\frac{1}{c_{1} \ell} \leq\left|v_{\ell}\right| \leq \frac{c_{1}}{\ell}$ and $\left|\Im\left(v_{\ell}\right)\right| \geq \frac{\left|\Re\left(v_{\ell}\right)\right|}{c_{1}}$, it suffices to show that $\mu_{x_{0}}$ is invariant under $n_{v_{\ell}}$ for all $\ell>\ell_{0}$. Note that $x_{\ell} \in E_{\rho}(\psi)$. Set $N_{0}:=\left\{n \in N: x_{\ell} n \in E_{\rho}(\psi)\right\}$. We have for any $n \in N_{0}$ and $f \in C_{c}(X)$,

$$
\frac{\mu_{x_{\ell}}(n . f)}{\mu_{x_{\ell}}(n . \psi)}=\lim _{T} \frac{\int_{0}^{T} f\left(x_{\ell} u_{t} n\right) d t}{\int_{0}^{T} \psi\left(x_{\ell} u_{t} n\right) d t}=\frac{\mu_{x_{\ell} n}(f)}{\mu_{x_{\ell} n}(\psi)} .
$$


On the other hand, by Proposition 8.7, we have

$$
\frac{\mu_{x_{\ell}}(n . f)}{\mu_{x_{\ell}}(n . \psi)}=\frac{\mu_{x_{\ell} n_{v_{\ell}}}(n . f)}{\mu_{x_{\ell} \cdot n_{v_{\ell}}}(n . \psi)}=\frac{\mu_{x_{\ell} n}\left(n_{v_{\ell}} \cdot f\right)}{\mu_{x_{\ell} . n}\left(n_{v_{\ell}} \cdot \psi\right)} .
$$

Therefore for any $n \in N_{0}$,

$$
\frac{\mu_{x_{\ell} n}(f)}{\mu_{x_{\ell} n}\left(n_{v_{\ell}} \cdot f\right)}=\frac{\mu_{x_{\ell} n}(\psi)}{\mu_{x_{\ell} \cdot n}\left(n_{v_{\ell}} \cdot \psi\right)}(\neq 0) .
$$

As $x_{\ell} \in E^{\prime}$, it follows from the definition of $E^{\prime}$ that we can take a sequence $n_{m}$ such that $x_{\ell} n_{m} \in E_{\rho}(\psi) \cap B\left(x_{0}, m^{-1}\right)$ and hence $x_{\ell} n_{m} \rightarrow x_{0}$ as $m \rightarrow \infty$.

In particular,

$$
\frac{\mu_{x_{0}}(f)}{\mu_{x_{0}}\left(n_{v_{\ell}} \cdot f\right)}=\lim _{m \rightarrow \infty} \frac{\mu_{x_{\ell} n_{m}}(f)}{\mu_{x_{\ell} n_{m}}\left(n_{v_{\ell}} \cdot f\right)}=\lim _{m \rightarrow \infty} \frac{\mu_{x_{\ell} n_{m}}(\psi)}{\mu_{x_{\ell} n_{m}}\left(n_{v_{\ell}} \cdot \psi\right)}=\frac{\mu_{x_{0}}(\psi)}{\mu_{x_{0}}\left(n_{v_{\ell}} \cdot \psi\right)} .
$$

It follows that $\mu_{x_{0}}$ and $n_{v_{\ell}} \cdot \mu_{x_{0}}$ are not mutually singular to each other. Hence by Lemma 8.2, $\mu_{x_{0}}=n_{v_{\ell}} \cdot \mu_{x_{0}}$.

Finally, we state the following: recall the notation $m_{N_{0}}^{\mathrm{BR}}$ from the subsection 2.5 .

Theorem 8.9. If $U_{0}$ is a one-parameter unipotent subgroup of $G$ and $\Gamma$ is a convex cocompact subgroup with $\delta>1$, then $m_{N_{0}}^{\mathrm{BR}}$ is $U_{0}$-ergodic for $N_{0}=C_{G}\left(U_{0}\right)$.

Proof. Let $k_{0} \in K$ be such that $U_{0}=k_{0}^{-1} U k_{0}$. If $B \subset X$ is a Borel subset which is $U_{0}$ invariant, then $B k_{0}$ is $U$-invariant. Hence by Corollary $8.3, m^{\mathrm{BR}}\left(B k_{0}\right)=0$ or $m^{\mathrm{BR}}\left(X-B k_{0}\right)=0$. By the definition of $m_{N_{0}}^{\mathrm{BR}}$, it follows that $m_{N_{0}}^{\mathrm{BR}}(B)=0$ or $m_{N_{0}}^{\mathrm{BR}}(X-B)=0$.

\section{BR IS NOT ERGODIC IF $0<\delta \leq 1$}

Let $\Gamma$ be a non-elementary torsion-free discrete subgroup of $G$. In this final section, we show that $m^{\mathrm{BR}}$ is never $U$-ergodic if $\Gamma$ is convex cocompact and $\delta \leq 1$.

By the Hopf decomposition theorem (cf. [11]), any ergodic measure preserving flow on a $\sigma$-finite measure space is either completely dissipative or completely conservative. In the former case, the action is isomorphic to the translation action of $\mathbb{R}$ on $\mathbb{R}$ with respect to the Lebesgue measure [1, Since $\Gamma$ is non-elementary, it follows that if $m^{\mathrm{BR}}$ were $U$-ergodic, then it must be completely conservative.

We first consider Fuchian groups: $\Gamma$ is called Fuchsian if it is contained in a conjugate of $\mathrm{PSL}_{2}(\mathbb{R})$. For a Fuchsian group $\Gamma, \Gamma$ is geometrically finite if and only if it is finitely generated.

Theorem 9.1. If $\Gamma$ is a finitely generated Fuchsian group, then $m^{\mathrm{BR}}$ is not $U$-ergodic.

Proof. The support of $\tilde{m}^{\mathrm{BR}}$ consists of $x n_{z} m_{\theta}$ on the unstable horospheres $H(x)$ based on $\Lambda(\Gamma)$, i.e., $x \in G$ with $x^{-} \in \Lambda(\Gamma), z \in \mathbb{C}$ and $m_{\theta} \in M$. As $\Gamma$ is Fuchsian, the convex hull of $\Lambda(\Gamma)$ is contained in a geodesic plane, say, $H$, preserved by $\Gamma$. Let $d$ denote a right $K$-invariant and left $G$-invariant metric on $G$. Then $x n_{z} m_{\theta} u_{t}=x n_{z+t e^{i \theta}} m_{\theta}$ and hence $d\left(x n_{z} m_{\theta} u_{t}, H\right)=d\left(x n_{z+t e^{i \theta}}, H\right) \rightarrow \infty$ as $t \rightarrow$ $\infty$, except for two directions of $\theta$ parallel to $H$. Since $H$ is $\Gamma$-invariant, we have $d\left(\gamma x n_{z+t e^{i \theta}}, H\right)=d\left(x n_{z+t e^{i \theta}}, H\right)$ for any $\gamma \in \Gamma$. Therefore for any $z \in \mathbb{C}$ and $\theta$ not parallel to $H, \Gamma \backslash \Gamma x n_{z} m_{\theta} u_{t} \rightarrow \infty$. This implies that almost all $m^{\mathrm{BR}}$-points $w \in X$, $w u_{t}$ goes to $\infty$ as $t \rightarrow \infty$. Therefore $m^{\mathrm{BR}}$ cannot be completely conservative for the $U$ action and hence is not $U$-ergodic. 
To prove the non-ergodicity in the remaining cases we begin by recalling some standard facts. For a 1 -set $\Lambda$ in the plane (see 6.1 for the definition), $\Lambda$ is called purely unrectifiable if $\mathcal{H}^{1}(\Lambda \cap \mathcal{C})=0$ for every rectifiable curve $\mathcal{C}$. We will use the following:

Theorem 9.2. (cf. [8, Theorem 6.4] or [21]) If $\Lambda \subset \mathbb{R}^{2}$ has Hausdorff dimension $\delta \leq 1$, then the orthogonal projection of $\Lambda$ on a.e. direction has also Hausdorff dimension $\delta$. Moreover if $\Lambda \subset \mathbb{R}^{2}$ is a 1-set which is purely unrectifiable, then the orthogonal projection of $\Lambda$ on a.e. direction has zero Lebesgue length.

Our proof in the non-Fuchsian case follows much the same philosophy that the $\mathrm{BR}$ measure and the BMS measure are very closely related. To be more precise, Theorem 9.2 and the definition of the BMS-measure imply that the BMS measure is not recurrent when $0<\delta<1$. We will use this fact to show the somewhat weaker non-recurrence holds for the BR measure. The following makes this more precise.

Definition 9.3. A measure preserving flow $u_{t}$ on a $\sigma$-finite measure space $(X, \mu)$ is called strongly recurrent if for any two measurable subsets $A_{1}, A_{2}$ with $\mu\left(A_{i}\right)>0$ we have $\left\{t: x u_{t} \in A_{2}\right\}$ is unbounded for $\mu$-a.e. $x \in A_{1}$.

Note that by the Hopf ratio ergodic theorem, any conservative, ergodic measure preserving flow is strongly recurrent. In particular, since $\Gamma$ is non-elementary, it follows that if $m^{\mathrm{BR}}$ were $U$-ergodic, then it must be strongly recurrent.

Theorem 9.4. Let $\Gamma$ be convex cocompact. Suppose either that $0<\delta<1$ or that $\Lambda(\Gamma)$ is a purely unrectifiable 1-set. Then the action of $U=\left\{u_{t}\right\}$ on $X=\Gamma \backslash G$ is not strongly recurrent for $m^{\mathrm{BR}}$. In particular $m^{\mathrm{BR}}$ is not $U$-ergodic.

Proof. We will prove this by contradiction. So, let us assume that the action of $U$ is strongly recurrent. We fix a BMS box $E=x_{0} B_{\rho}$ in $X$ with small $0<\rho \ll 1$. We claim that there exists a Borel subset $E^{\prime}$ of $E \cap \operatorname{supp}\left(m^{\mathrm{BR}}\right)$ with $m^{\mathrm{BR}}\left(E-E^{\prime}\right)=0$ such that for any $x \in E^{\prime},\left\{t \in \mathbb{R}: x n u_{t} \in E\right\}$ is unbounded for almost all $n \in N$ (with respect to the Lebesgue measure of $N$ ).

To show this, for any $\ell \in \mathbb{N}$, let $\varepsilon(\ell)=\varepsilon(\ell, E)>0$ be chosen so that if we let $B_{\ell}=\check{N}_{\varepsilon(\ell)} A_{\varepsilon(\ell)} N_{\ell} M$, then the map $b \mapsto x g b$ is injective on $B_{\ell}$ for all $x \in E$ and all $g$ in the $\rho$-neighborhood of $e$ in $G$. This is possible as $\Gamma$ does not contain any parabolic element. Recalling the notation $T_{\varepsilon(\ell)}=\check{N}_{\varepsilon(\ell)} A_{\varepsilon(\ell)} M$, let $\left\{x_{j} T_{\varepsilon(\ell)} N_{\varepsilon(\ell)}: j \in J_{\ell}\right\}$ be a finite cover of $E$. Then, the assumption on the strong recurrence and the Fubini theorem imply that for any $j \in J_{\ell}$ and almost every $x \in x_{j} T_{\varepsilon(\ell)} N_{\varepsilon(\ell)}$, we have that for a. e. $n \in N_{\ell}$, the set $\left\{t: x n u_{t} \in E\right\}$ is unbounded. Hence, the claim follows.

Fix $x^{\prime} \in E^{\prime}$ and consider a sequence $x^{\prime} b_{\ell}$ for $b_{\ell}=a_{\log (\rho / \ell)}, \ell \in \mathbb{N}$. Since $\left(x^{\prime}\right)^{-} \in \Lambda(\Gamma)$ there exists a subsequence $\ell_{i} \rightarrow+\infty$ such that $\left\{x^{\prime} b_{\ell_{i}}\right\}$ converges to some $y^{\prime} \in \Omega$. As $\left(y^{\prime}\right)^{+} \in \Lambda(\Gamma),\left.\mu_{y^{\prime}}^{\mathrm{PS}}\right|_{y^{\prime} N_{\rho}}$ is a positive $\delta$-dimensional Hausdorff measure. By the assumption, Theorem 6.3 and Theorem 9.2 imply that for almost all $m_{\theta} \in M$ we have $p_{\theta}\left(y^{\prime} N_{\rho} \cap \operatorname{supp}\left(\mu_{y^{\prime}}^{\mathrm{PS}}\right)\right)$ has zero Lebesgue length (see Section 5.1 for the notation $p_{\theta}$ ). Since $E$ is $M$-invariant and $E^{\prime}$ is of full measure in $E$, we can choose $m_{\theta} \in M$ such that $x^{\prime} m_{\theta} \in E^{\prime}$ and $p_{\theta}\left(y^{\prime} N_{\rho} \cap \operatorname{supp}\left(\mu_{y^{\prime}}^{\mathrm{PS}}\right)\right)$ has zero Lebesgue length. We set

$$
x=x^{\prime} m_{\theta}, x_{\ell_{i}}:=x^{\prime} b_{\ell_{i}} m_{\theta}=x^{\prime} m_{\theta} b_{\ell_{i}} \text {, and } y=y^{\prime} m_{\theta} .
$$

Then $x_{\ell_{i}} \rightarrow y$ as $\ell_{i} \rightarrow+\infty,\left\{t: x n u_{t} \in E\right\}$ is unbounded for a.e. $n \in N$ and the projection $p_{0}\left(y N_{\rho} \cap \operatorname{supp}\left(\mu_{y}^{\mathrm{PS}}\right)\right)$ has zero Lebesgue length. 
By the Fubini theorem, there exists a Borel subset $V^{\prime} \subset V$ of co-measure zero that for each $v \in V^{\prime}$, the set $\left\{t: x v u_{t} \in E\right\}$ is unbounded. Hence, for each $j \in \mathbb{Z}$, we can find a sequence $v_{j}^{\prime} \in V^{\prime} \cap\left\{n_{i t}: t \in[j-1, j+1]\right\}$. With abuse of notation, we consider $v_{j}^{\prime}$ as an element of $\mathbb{R}$ and write $v_{j}^{\prime} \in[j-1, j+1]$.

Since $E=x_{0} B_{\rho}$ with $x_{0}^{ \pm} \in \Lambda(\Gamma)$, the condition $g n_{z} \in E$ with $g^{-} \in \Lambda(\Gamma)$ implies that $g n_{z+w} \in \Omega$ for some $w \in \mathbb{C}$ with $|w| \leq \rho$. Therefore, for each $v_{j}^{\prime} \in V^{\prime}$, $x v_{j}^{\prime} u_{t} \in E$ for some $t \in \mathbb{R}$ implies the existence of $w \in \mathbb{C}$ with $|w| \leq \rho$ such that $x v_{j}^{\prime} u_{t} n_{w} \in \Omega$. This in particular implies that for each $j \in \mathbb{Z}$, there exists $x v_{j} \in x V \cap p_{0}\left(\operatorname{supp}\left(\mu_{x}^{\mathrm{PS}}\right)\right)$ with $\left|v_{j}-v_{j}^{\prime}\right| \leq \rho \leq 1$. Note that $v_{j} \in[j-2, j+2]$ for each $j \in \mathbb{Z}$.

Flowing $x v_{j}$ by $b_{\ell}$, we get $x v_{j} b_{\ell}=x_{\ell}\left(b_{\ell}^{-1} v_{j} b_{\ell}\right)$. Setting $v_{j}^{\ell}:=\left(b_{\ell}^{-1} v_{j} b_{\ell}\right) \in V$, we have

$$
\frac{(j-2) \rho}{\ell} \leq v_{j}^{\ell} \leq \frac{(j+2) \rho}{\ell} \quad \text { and } \quad v_{j}^{\ell} \in p_{0}\left(\operatorname{supp}\left(\mu_{x_{\ell}}^{\mathrm{PS}}\right)\right) .
$$

Therefore $x_{\ell} V_{\rho} \cap p_{0}\left(\operatorname{supp}\left(\mu_{x_{\ell}}^{\mathrm{PS}}\right)\right)$ contains $x_{\ell} v_{j}^{\ell}$ whenever $\frac{|j \pm 2|}{\ell}<1$.

Note that $x_{\ell_{i}} N_{\rho} \cap \operatorname{supp}\left(\mu_{x_{\ell_{i}}}^{\mathrm{PS}}\right)=\left\{x_{\ell_{i}} n_{z}:|z|<\rho,\left(x_{\ell_{i}} n_{z}\right)^{+} \in \Lambda(\Gamma)\right\}$ and that any limit of $x_{\ell_{i}} n$ is of the form $y n$ as $x_{\ell_{i}} \rightarrow y$. Since the visual map, $g \mapsto g^{+}$, is continuous and $\Lambda(\Gamma)$ is closed, it follows that the sequence of subsets $x_{\ell_{i}} N_{\rho} \cap$ $\operatorname{supp}\left(\mu_{x_{\ell_{i}}}^{\mathrm{PS}}\right)$ converges to $y N_{\rho} \cap \operatorname{supp}\left(\mu_{y}^{\mathrm{PS}}\right)$ in the sense that if $w_{i} \in x_{\ell_{i}} N_{\rho} \cap \operatorname{supp}\left(\mu_{x_{\ell_{i}}}^{\mathrm{PS}}\right)$ converges to $w$, then $w \in y N_{\rho} \cap \operatorname{supp}\left(\mu_{y}^{\mathrm{PS}}\right)$. Therefore, as $i \rightarrow \infty, p_{0}\left(x_{\ell_{i}} N_{\rho} \cap\right.$ $\left.\operatorname{supp}\left(\mu_{x_{\ell_{i}}}^{\mathrm{PS}}\right)\right)$ converges to $p_{0}\left(y N_{\rho} \cap \operatorname{supp}\left(\mu_{y}^{\mathrm{PS}}\right)\right)$ as well in the similar sense as above. Thus we have obtained that $y V_{\rho} \subset p_{0}\left(\operatorname{supp}\left(\mu_{y}^{\mathrm{PS}}\right)\right)$ and hence the Lebesgue length of $p_{0}\left(N_{\rho} \cap \operatorname{supp}\left(\mu_{y}^{\mathrm{PS}}\right)\right)$ is positive, yielding a contradiction.

A Fuchsian group $\Gamma$ is called of the first kind if $\Lambda(\Gamma)$ is a great circle and of the second kind otherwise. By Canary and Taylor [6], any torsion-free and convex cocompact group $\Gamma$ with $\delta=1$ is either a Fuchsian subgroup of the first kind or a quasi-conformal conjugation of a Fuchsian group of the second kind, and in the latter case, the limit set $\Lambda(\Gamma)$ is totally disconnected.

We thank Chris Bishop for providing the proof of the following theorem:

Theorem 9.5 (Bishop). If the limit set of a convex cocompact Kleinian group is totally disconnected, it is always purely unrectifiable.

Proof. If the limit set of a convex co-compact group hits a rectifiable curve in positive length, then the limit set contains a circle (or line). This can be proved by taking a sequence of balls shrinking to a point of density of the set of positive length that is also a point of tangency for the rectifiable curve and rescaling the balls by group elements to approximately unit size (this can always be done in a convex co-compact group). The rescaled sets must have a subsequence that converges to a line segment or circular arc in the limit set. Rescaling of the arc leads to a full circle or line in the limit set.

In particular, a convex co-compact limit set that is totally disconnected cannot hit any rectifiable curve in positive length. Thus, it is purely unrectifiable.

Therefore by Theorem 9.1, Theorem 9.4, and Theorem 9.5, we have:

Theorem 9.6. Let $\Gamma$ be torsion-free and convex cocompact with $0<\delta \leq 1$. Then $m^{\mathrm{BR}}$ is not $U$-ergodic. 


\section{ACKNOWLEDGMENTS}

We are very grateful to Tim Austin for numerous helpful discussions regarding various aspects of this project. We also thank Chris Bishop and Edward Taylor for helpful correspondences regarding totally disconnected limit sets of Kleinian groups.

\section{REFERENCES}

[1] Jon Aaronson, An introduction to infinite ergodic theory, Mathematical Surveys and Monographs, vol. 50, American Mathematical Society, Providence, RI, 1997. MR1450400 (99d:28025)

[2] Martine Babillot, On the mixing property for hyperbolic systems, Israel J. Math. 129 (2002), 61-76, DOI 10.1007/BF02773153. MR1910932 (2003g:37008)

[3] M. Brin, Ergodic theory of frame flows, Ergodic theory and dynamical systems, II (College Park, Md., 1979/1980), Progr. Math., vol. 21, Birkhäuser, Boston, Mass., 1982, pp. 163-183. MR670078 (83m:58059)

[4] B. H. Bowditch, Geometrical finiteness for hyperbolic groups, J. Funct. Anal. 113 (1993), no. 2, 245-317, DOI 10.1006/jfan.1993.1052. MR.1218098(94e:57016)

[5] Marc Burger, Horocycle flow on geometrically finite surfaces, Duke Math. J. 61 (1990), no. 3, 779-803, DOI 10.1215/S0012-7094-90-06129-0. MR:1084459 (91k:58102)

[6] Richard D. Canary and Edward Taylor, Kleinian groups with small limit sets, Duke Math. J. 73 (1994), no. 2, 371-381, DOI 10.1215/S0012-7094-94-07316-X. MR1262211 (94m:57028)

[7] M. Einsiedler and E. Lindenstrauss, Diagonal actions on locally homogeneous spaces, Homogeneous flows, moduli spaces and arithmetic, Clay Math. Proc., vol. 10, Amer. Math. Soc., Providence, RI, 2010, pp. 155-241. MR2648695 (2011f:22026)

[8] Kenneth Falconer, Fractal geometry, 2nd ed., John Wiley \& Sons, Inc., Hoboken, NJ, 2003. Mathematical foundations and applications. MR2118797 (2006b:28001)

[9] L. Flaminio and R. J. Spatzier, Geometrically finite groups, Patterson-Sullivan measures and Ratner's rigidity theorem, Invent. Math. 99 (1990), no. 3, 601-626, DOI 10.1007/BF01234433. MR.1032882(91d:58201)

[10] Michael Hochman, A ratio ergodic theorem for multiparameter non-singular actions, J. Eur. Math. Soc. (JEMS) 12 (2010), no. 2, 365-383, DOI 10.4171/JEMS/201. MR2608944 (2011g:37006)

[11] E Hopf, Ergodentheorie, Ergebnisse der Mathematik Number 5 (1937).

[12] Xiaoyu $\mathrm{Hu}$ and S. James Taylor, Fractal properties of products and projections of measures in $\mathbf{R}^{d}$, Math. Proc. Cambridge Philos. Soc. 115 (1994), no. 3, 527-544, DOI 10.1017/S0305004100072285. MR1269937 (95f:28013)

[13] A. Katok and R. J. Spatzier, Invariant measures for higher-rank hyperbolic abelian actions, Ergodic Theory Dynam. Systems 16 (1996), no. 4, 751-778, DOI 10.1017/S0143385700009081. MR1406432 (97d:58116)

[14] Ulrich Krengel, Ergodic theorems, de Gruyter Studies in Mathematics, vol. 6, Walter de Gruyter \& Co., Berlin, 1985. With a supplement by Antoine Brunel. MR797411 (87i:28001)

[15] Elon Lindenstrauss, Invariant measures and arithmetic quantum unique ergodicity, Ann. of Math. (2) 163 (2006), no. 1, 165-219, DOI 10.4007/annals.2006.163.165. MR2195133 (2007b:11072)

[16] J.-L. Lions and E. Magenes, Non-homogeneous boundary value problems and applications. Vol. I, Springer-Verlag, New York-Heidelberg, 1972. Translated from the French by P. Kenneth; Die Grundlehren der mathematischen Wissenschaften, Band 181. MR0350177 $(50$ \#2670)

[17] G. A. Margulis, On the action of unipotent groups in the space of lattices, Lie groups and their representations (Proc. Summer School, Bolyai, János Math. Soc., Budapest, 1971), Halsted, New York, 1975, pp. 365-370. MR0470140 (57 \#9907)

[18] G. A. Margulis, Indefinite quadratic forms and unipotent flows on homogeneous spaces, Dynamical systems and ergodic theory (Warsaw, 1986), Banach Center Publ., vol. 23, PWN, Warsaw, 1989, pp. 399-409. MR1102736 (92g:11034)

[19] J. M. Marstrand, Some fundamental geometrical properties of plane sets of fractional dimensions, Proc. London Math. Soc. (3) 4 (1954), 257-302. MR0063439 (16,121g) 
[20] Pertti Mattila, Geometry of sets and measures in Euclidean spaces, Cambridge Studies in Advanced Mathematics, vol. 44, Cambridge University Press, Cambridge, 1995. Fractals and rectifiability. MR 1333890 (96h:28006)

[21] Pertti Mattila, Hausdorff dimension, projections, and the Fourier transform, Publ. Mat. 48 (2004), no. 1, 3-48, DOI 10.5565/PUBLMAT_48104_01. MR2044636(2004k:28018)

[22] Calvin C. Moore, Ergodicity of flows on homogeneous spaces, Amer. J. Math. 88 (1966), 154-178. MR0193188 (33 \#1409)

[23] Calvin C. Moore, Ergodicity of flows on homogeneous spaces, Amer. J. Math. 88 (1966), 154-178. MR0193188 (33 \#1409)

[24] Hee Oh and Nimish A. Shah, Equidistribution and counting for orbits of geometrically finite hyperbolic groups, J. Amer. Math. Soc. 26 (2013), no. 2, 511-562, DOI 10.1090/S0894-03472012-00749-8. MR3011420

[25] Jean-Pierre Otal and Marc Peigné, Principe variationnel et groupes kleiniens (French, with English and French summaries), Duke Math. J. 125 (2004), no. 1, 15-44, DOI 10.1215/S00127094-04-12512-6. MR2097356 (2005h:37053)

[26] S. J. Patterson, The limit set of a Fuchsian group, Acta Math. 136 (1976), no. 3-4, 241-273. MR0450547(56 \#8841)

[27] Marc Peigné, On the Patterson-Sullivan measure of some discrete group of isometries, Israel J. Math. 133 (2003), 77-88, DOI 10.1007/BF02773062. MR1968423 (2004b:30079)

[28] Yuval Peres and Wilhelm Schlag, Smoothness of projections, Bernoulli convolutions, and the dimension of exceptions, Duke Math. J. 102 (2000), no. 2, 193-251, DOI 10.1215/S00127094-00-10222-0. MR:1749437 (2001d:42013)

[29] Marina Ratner, On measure rigidity of unipotent subgroups of semisimple groups, Acta Math. 165 (1990), no. 3-4, 229-309, DOI 10.1007/BF02391906. MR1075042(91m:57031)

[30] Marina Ratner, On Raghunathan's measure conjecture, Ann. of Math. (2) 134 (1991), no. 3, 545-607, DOI 10.2307/2944357. MR1135878 (93a:22009)

[31] Thomas Roblin, Ergodicité et équidistribution en courbure négative (French, with English and French summaries), Mém. Soc. Math. Fr. (N.S.) 95 (2003), vi+96. MR2057305(2005d:37060)

[32] Thomas Roblin, Sur l'ergodicité rationnelle et les propriétés ergodiques du flot géodésique dans les variétés hyperboliques (French, with French summary), Ergodic Theory Dynam. Systems 20 (2000), no. 6, 1785-1819, DOI 10.1017/S0143385700000997. MR1804958 (2001m:37061)

[33] Daniel J. Rudolph, Ergodic behaviour of Sullivan's geometric measure on a geometrically finite hyperbolic manifold, Ergodic Theory Dynam. Systems 2 (1982), no. 3-4, 491-512 (1983), DOI 10.1017/S0143385700001735. MR721736 (85i:58101)

[34] Dennis Sullivan, The density at infinity of a discrete group of hyperbolic motions, Inst. Hautes Études Sci. Publ. Math. 50 (1979), 171-202. MR556586 (81b:58031)

[35] Dennis Sullivan, Entropy, Hausdorff measures old and new, and limit sets of geometrically finite Kleinian groups, Acta Math. 153 (1984), no. 3-4, 259-277, DOI 10.1007/BF02392379. MR766265 (86c:58093)

[36] D Winter, Mixing of frame flow for rank one locally symmetric spaces and measure classification, available at arXiv: 1403.2425.

[37] Shing Tung Yau, Harmonic functions on complete Riemannian manifolds, Comm. Pure Appl. Math. 28 (1975), 201-228. MR0431040 (55 \#4042)

[38] R. Zweimüller, Hopf's ratio ergodic theorem by inducing. Preprint.

Department of Mathematics, The University of Texas at Austin, Austin, Texas 78750

E-mail address: amir@math.utexas.edu

Department of Mathematics, Yale University, New Haven, Connecticut 06520 and

Korea Institute for Advanced Study, Seoul, Korea

E-mail address: hee.oh@yale.edu 